\title{
A Duality Approach to Continuous-Time Contracting Problems with Limited Commitment*
}

\author{
Jianjun Miao $^{\dagger} \quad$ Yuzhe Zhang ${ }^{\ddagger}$
}

September 10, 2014

\begin{abstract}
We propose a duality approach to solving contracting models with either one-sided or two-sided limited commitment in continuous time. We establish weak and strong duality theorems and provide a dynamic programming characterization of the dual problem. The dual problem gives a linear Hamilton-Jacobi-Bellman equation with a known state space subject to free-boundary conditions, making analysis much more tractable than the primal problem. We provide two explicitly solved examples of a consumption insurance problem. We characterize the optimal consumption allocation in terms of the marginal utility ratio. We find that neither autarky nor full risk sharing can be an optimal contract with two-sided limited commitment, unlike in discrete-time models. We also derive an explicit solution for the unique long-run stationary distribution of consumption relative to income.
\end{abstract}

JEL Classification: C61, D86, D91, E21

Keywords: continuous-time contracts, limited commitment, risk sharing, duality, dynamic programming, regulated Brownian motion

\footnotetext{
${ }^{*}$ We thank Yuliy Sannikov, Harald Uhlig, and Noah Williams for helpful comments. We also thank the editor (Alessandro Pavan), an associate editor and two anonymous reviewers for thoughtful and helpful comments and suggestions.

${ }^{\dagger}$ Department of Economics, Boston University, 270 Bay State Road, Boston, MA 02215, USA, CEMA, Central University of Finance and Economics, and AFR, Zhejiang University, China. Email: miaoj@bu.edu. Tel: (617) 3536675 .

${ }^{\ddagger}$ Department of Economics, Texas A\&M University, College Station, TX, 77843. Email: yuzhezhang@econmail.tamu.edu. Tel.: 319-321-1897.
} 


\section{Introduction}

Many empirical studies find that idiosyncratic variation in consumption is systematically related to idiosyncratic variation in income, rejecting the hypothesis of full risk sharing (e.g., Cochrane (1991), Mace (1991), and Townsend (1994)). Instead of assuming exogenous market incompleteness, one important approach to reconciling this empirical evidence is to assume that individuals have limited commitment (e.g., Kocherlakota (1996), Alvarez and Jermann (2000), and Ligon, Thomas, and Worrall (2002)). This assumption is motivated by the fact that debt repayments are costly to enforce. Debt collection, litigation, and income garnishment are costly, and the debtor may default on debt. In this case, individual income risks are only incompletely shared.

Although discrete-time dynamic models with limited commitment have been widely applied in economics and finance, ${ }^{1}$ these models are typically difficult to solve analytically and numerical solutions are needed. The main contribution of this paper is to propose a duality approach in a continuous-time setup, which permits analytical solutions. We consider a consumption insurance problem between a principal and an agent analogous to the problems analyzed by Thomas and Worrall (1988), Kocherlakota (1996), Alvarez and Jermann (2000), Ligon, Thomas, and Worrall (2002), and Ljungqvist and Sargent (2004). ${ }^{2}$ The continuous-time setup is analytically convenient and allows us to derive sharp and transparent results. We find that the usual dynamic programming approach using the agent's continuation value as a state variable in the primal problem delivers a nonlinear Hamilton-Jacobi-Bellman (HJB) equation with state constraints. The state space of the continuation value is endogenous in models with two-sided limited commitment. Such a nonlinear HJB equation typically does not admit any analytical solution and is difficult to analyze even numerically. By contrast, the dual problem transforms the primal problem with participation constraints into an unconstrained problem, which delivers a linear HJB equation subject to free-boundary conditions. Technically, it is an instantaneous (or singular) control problem, similar to those analyzed in Harrison and Taksar (1983), Harrison (1985), and Stokey (2008).

We study the link between the dual and primal problems and establish the weak and strong

\footnotetext{
${ }^{1}$ Other examples include applications to wage contracts by Thomas and Worrall (1988), sovereign debt by Bulow and Rogoff (1989), Kletzer and Wright (2000), and Hellwig and Lorenzoni (2009), asset markets by Kehoe and Levine (1993) and Alvarez and Jermann (2000, 2001), optimal taxation by Chari and Kehoe (1993), business cycles by Cooley, Marimon, and Quadrini (2004), international business cycles by Kehoe and Perri (2002), consumption inequality by Krueger and Uhlig (2006) and Krueger and Perri (2006), the welfare effects of a progressive tax by Krueger and Perri (2011), political economy by Acemoglu, Golosov, and Tsyvinski (2011), and asset bubbles by Kocherlakota (2008) and Miao and Wang (2011, 2012).

${ }^{2}$ The principal and the agent can be interpreted in different ways in different contexts. They can be two households, a planner and a household, or a firm and a worker.
} 
duality theorems. We provide a dynamic programming characterization of the dual problem using the usual state variables (individual incomes) together with additional costate variables. The costate variables are the cumulative amounts of the Lagrange multipliers associated with the intertemporal participation constraints, starting from pre-specified initial conditions. These costate variables are nonnegative and increasing processes. They are also the control variables in the dual problem and rise whenever the participation constraints bind.

In the case of one-sided limited commitment, there is only one costate variable, which is associated with the agent's participation constraints. To facilitate discussion, we first consider the case in which the principal and the agent have an identical discount rate. In this case, the costate variable is also equal to the ratio of the marginal utilities of the principal and the agent. The agent's current income and the marginal utility ratio constitute the state variables of the HJB equation for the dual problem. From the HJB equation, we derive a free boundary using the agent's binding participation constraints. The free boundary partitions the state space into two regions: the jump region and the no-jump region. When the initial promised value to the agent is higher than the outside value, the initial state of the marginal utility ratio and the agent's income must lie in the no-jump region. Subsequently, the marginal utility ratio and the agent's consumption remain constant in the interior of the no-jump region. They rise instantaneously whenever the agent's income increases and hits the free boundary. The marginal utility ratio keeps the agent's continuation value above the outside option value.

If the principal and the agent have different discount rates, then the solution is similar to that in the case of equal discount rates except that we must adjust the costate variable by the difference in the discount rates so that the adjusted costate variable is equal to the marginal utility ratio. This ratio and the agent's consumption are no longer constant in the no-jump region. They rise (fall) over time when the agent is more (less) patient than the principal.

In the case of two-sided limited commitment, we suppose for simplicity that the principal and the agent have an identical discount rate. There are two costate variables associated with the principal's and the agent's participation constraints, respectively. These two costate variables and the agent's income constitute the state variables of the HJB equation for the dual problem. We show that the HJB equation is linearly homogeneous and can be reduced to a two-dimensional problem using the agent's income and the marginal utility ratio as state variables. The marginal utility ratio is also equal to a suitably defined ratio of the two costate variables. From the HJB equation, we solve for the two free boundaries using the binding participation constraints of the principal and the agent. The two free boundaries partition the state space into three areas. The area between the two free boundaries is the no-jump region. The other two areas are the jump region. When the initial promised value to the agent is higher 
than his outside value and also not too large to push the principal's value below the principal's outside value, the initial state of the marginal utility ratio and the agent's income must lie in the no-jump region. Subsequently, the marginal utility ratio and the agent's consumption remain constant. Whenever the agent's income rises (falls) and hits the boundary determined by the agent's (the principal's) binding participation constraints, the marginal utility ratio and the agent's consumption rise (fall) instantaneously. The state processes of the marginal utility ratio and the agent's income will never move out of the no-jump region.

Another main contribution of this paper is to provide two explicitly solved examples with either one-sided or two-sided limited commitment. This contribution is important because, to the best of our knowledge, our paper is the first one that derives explicit closed-form solutions for dynamic models with two-sided limited commitment. Furthermore, our explicitly solved examples exhibit different risk-sharing dynamics than those in the discrete-time models. In particular, neither autarky nor full risk sharing can be an optimal contract in our examples.

The first example is a continuous-time version of the discrete-time models analyzed in Thomas and Worrall (1988), Krueger and Uhlig (2006), and in Chapter 19 of Ljungqvist and Sargent (2004). In this example, the principal is a risk-neutral planner and the agent is a household with a constant relative risk aversion utility function. Only the agent has limited commitment and may renege on the contract and enter autarky. We assume that the agent's income follows a geometric Brownian motion process and that the agent and the principal may have different subjective discount factors. In their discrete-time model, Ljungqvist and Sargent (2004) assume that the agent and the principal have an identical discount factor and show that the agent will be fully insured in the long run when his income follows an independently and identically distributed (IID) process with a finite state space. ${ }^{3}$ By contrast, in our continuoustime model, the agent can never be fully insured because the income process is unbounded and the agent's consumption increases whenever his income rises to a new highest level to date. ${ }^{4}$ We show that the log consumption-income ratio is a one-sided regulated Brownian motion with a lower barrier (Harrison (1985) and Stokey (2008)). It has a unique long-run stationary distribution with an unbounded support, if the agent's income growth is sufficiently large.

In the second example, we incorporate the principal's limited commitment into the first example. ${ }^{5}$ We suppose that the principal may also renege on the contract and take the autarky

\footnotetext{
${ }^{3}$ In a discrete-time model, Zhang (2013) allows the agent and the principal to have different subjective discount factors and provides a stopping time characterization of the optimal contract. But his approach does not admit an explicit solution.

${ }^{4}$ Even when the income process lives in a bounded interval, the first-best allocation may not be attainable under one-sided limited commitment. For example, this happens if the upper bound of that interval cannot be attained and if the initial promised value to the agent is the autarky value. But the optimal contract may converge to the first best.

${ }^{5}$ In Appendix C, we modify the second example by considering a symmetric setup in which both the principal
} 
value of zero. This problem is a continuous-time version of the problems analyzed in Kocherlakota (1996), Alvarez and Jermann (2000), Ligon, Thomas, and Worrall (2002), and Chapter 20 of Ljungqvist and Sargent (2004). In a symmetric setup, Kocherlakota (1996) shows that the agent's consumption has a unique long-run stationary distribution when incomes follow an IID process with a finite state space. By contrast, in our continuous-time model with a geometric Brownian motion income process, consumption itself has no long-run stationary distribution. But the log consumption-income ratio has a unique stationary distribution with a bounded support. The log consumption-income ratio is a two-sided regulated Brownian motion with two finite barriers (Harrison (1985) and Stokey (2008)). We call the interval between these two barriers the risk-sharing band. Under full risk sharing, consumption is constant and hence the band becomes the real line. The wider is the band, the more is the risk sharing.

In discrete-time models with two sided limited commitment, Kocherlakota (1996), Alvarez and Jermann (2000), and Ligon, Thomas, and Worrall (2002) show that, depending on parameter values, there are three cases for an optimal contract: full risk sharing, autarky (no risk sharing), and limited risk sharing. In particular, Ligon, Thomas, and Worrall (2002) show that when the discount factor is sufficiently small, autarky is the only sustainable allocation, and when the discount factor is sufficiently large, full risk sharing can be achieved. By contrast, in our continuous-time model, only limited risk sharing can happen. This result reflects the difference in the nature of shocks and the difference in the continuous-time and discrete-time frameworks. In discrete-time models, the state space of shocks is typically finite. In our model, the shock is driven by a Brownian motion. Full risk sharing cannot be an optimal contract in our model because the unbounded Brownian motion shock can cause the autarky value to exceed any constant utility level from full risk sharing. This result also holds in a discrete-time model if the income process is unbounded. ${ }^{6}$

Autarky cannot be an optimal contract in our model because the cost of staying in autarky is so high that participating in risk sharing is always mutually beneficial no matter how heavily the principal and the agent discount the future utility level. ${ }^{7}$ This result is not due to the nonstationarity or unboundedness of the income process used in our example because we show that autarky is the only optimal contract in a discrete-time approximation of our model if the

and the agent have an identical constant absolute risk aversion utility function. The agent's income is modeled as an arithmetic Brownian motion and the principal's income is the negative of the agent's income so that these two incomes are perfectly negatively correlated. In this case, we show that the consumption-income difference is a two-sided regulated Brownian motion with two barriers and has a unique long-run stationary distribution. We also obtain a comparative statics result similar to that in the second example.

${ }^{6}$ Note that even when the income process is bounded, the first best may not be attainable under two-sided limited commitment (see Kocherlakota (1996) or Ljungqvist and Sargent (2004) for a discussion).

${ }^{7}$ The intuition is subtle. See Section 3.3 and the proof of Proposition 3 for an analysis of a discrete-time version of our model. 
nonstationary income process is not too volatile or the principal and the agent are sufficiently impatient. In particular, we show that the net benefit from risk sharing depends on the length of the time interval. Thus, time frequency matters for the optimal contract.

We conduct a comparative statics analysis with respect to the agent's risk aversion parameter, the volatility of the income process, and the subjective discount rate. We find that the risk-sharing band expands when one of the following cases happens: (i) the subjective discount rate falls, (ii) the volatility of the income process rises, and (iii) the agent's coefficient of relative risk aversion rises. This result is intuitive. When contracting parties are more patient, cooperation and risk sharing are more likely to sustain. When either the income volatility or the degree of risk aversion is high, the autarky value is low, thereby reducing the agent's incentive to renege.

Related Literature The usual approach to solving dynamic contracting models is to use dynamic programming and adopt the agent's promised utility (or the continuation value) as a state variable. This approach is pioneered by Green (1987), Thomas and Worrall (1988), Spear and Srivastava (1987), and Abreu, Pearce, and Staccheti (1990). ${ }^{8}$ DeMarzo and Sannikov (2006), Biais, Mariotti, Plantin, and Rochet (2007), Sannikov (2008), and Williams (2009, 2011) extend this approach to study continuous-time principal-agent problems with hidden action or hidden information. Miao and Rivera (2013) and Strulovici (2011) introduce robustness and renegotiation-proofness into this framework, respectively. Grochulski and Zhang (2011) apply this approach to study a consumption insurance problem with one-sided limited commitment in continuous time. They provide an explicit solution to the problem when the principal and the agent are equally patient. However, their analysis cannot be generalized to more general discount rates or to the case with two-sided limited commitment.

Our duality approach is closely related to that in the discrete-time setup proposed by Marcet and Marimon $(1992,1998)$, who build on the early work of Hansen, Epple, and Roberds (1985) and Kydland and Prescott (1980). The Marcet-Marimon approach has been extended by Messner, Pavoni, and Sleet $(2011,2012)$ in more general discrete-time contracting problems. Their extension can handle contracting problems with hidden information, hidden action, or limited commitment. They formulate dual problems and provide iterative convergent algorithms. Our paper focuses on problems with limited commitment. Unlike their discrete-time setup, our continuous-time approach allows us to derive transparent results and closed-form solutions. Technically, our continuous-time setup also requires different mathematical machinery and our results of the weak and strong duality theorems are nontrivial. In particular, our analysis uses

\footnotetext{
${ }^{8}$ Chapters 19 and 20 of Ljungqvist and Sargent (2004) provide an excellent introduction to this approach.
} 
the theory of regulated Brownian motion and singular control in Harrison (1985).

Our duality approach is also related to the mathematical finance literature on portfolio choice in continuous time (see, e.g., Xu and Shreve (1992), He and Pages (1993)). To the best of our knowledge, our paper is the first one to apply this approach to dynamic contracting problems with limited commitment in continuous time. Sannikov (2012) applies the duality approach to analyze a moral hazard model in which the agent's actions have long-run effects. His dual problem reduces to a standard optimal control problem rather than a singular control problem. As is well known, the duality approach is related to the maximum principle (e.g., Bismut (1973)). Williams (2009, 2011) applies the maximum principle to analyze the agent's incentive problems in models with hidden action or hidden information. Neither Sannikov (2012) nor Williams $(2009,2011)$ studies models with limited commitment.

Our characterization of the optimal consumption policy in terms of the marginal utility ratio is similar to that of Thomas and Worrall (1988) and Ligon, Thomas, and Worrall (2002). In a discrete-time model with two-sided limited commitment, they derive a simple updating rule in terms of the marginal utility ratio. Each state of nature is associated with a particular interval of possible ratios of marginal utilities. Given the current state and the previous period's marginal utility ratio, the new ratio lies within the interval associated with the current state, such that the change in the ratio is minimized. The updating rule requires that the ratio of marginal utilities be kept constant whenever possible. However, if full risk sharing is not attainable, then the ratio must change to an endpoint of the current interval, and one of the households will be constrained, i.e., its participation constraints bind. Although the updating rule is intuitive, the discrete-time setup does not permit an explicit solution to the intervals of marginal utility ratios. Thus, numerical solutions are needed and they get messy when there are many states of shocks.

In our continuous-time model, the marginal utility ratio is also kept constant whenever possible. There is a band for marginal utility ratios associated with each income level. When the agent's income is sufficiently high to hit a boundary such that the agent's participation constraints bind, the marginal utility ratio rises continuously. But when the agent's income is sufficiently low to hit another boundary such that the principal's participation constraints bind, the marginal utility ratio falls continuously. The marginal utility ratio always lies within the band and moves continuously. The analytical power of our duality approach is that we are able to explicitly characterize the two boundaries of the band and the stationary distribution of consumption relative to income. 


\section{One-Sided Limited Commitment}

Consider a canonical contracting model with limited commitment in a continuous-time infinitehorizon environment. We fix a filtered probability space $\left(\Omega, \mathcal{F},\left\{\mathcal{F}_{t}\right\}_{t \geq 0}, P\right)$ on which is defined a one-dimensional standard Brownian motion $\left\{B_{t}\right\}_{t \geq 0}$. The filtration $\left\{\mathcal{F}_{t}\right\}_{t \geq 0}$ is generated by this Brownian motion and $\mathcal{F}_{0}$ is trivial. For ease of exposition, we shall refer to the two contracting parties as the principal and the agent. The principal is risk neutral and discounts future cash flows at the rate $r>0$. The agent is risk averse and has an income process $Y=\left\{Y_{t}\right\}_{t \geq 0}$ satisfying the stochastic differential equation:

$$
d Y_{t}=\mu\left(Y_{t}\right) d t+\sigma\left(Y_{t}\right) d B_{t}, \quad Y_{0}=y
$$

where $\mu: \mathbb{R}_{+} \rightarrow \mathbb{R}$ and $\sigma: \mathbb{R}_{+} \rightarrow \mathbb{R}_{+}$.

Assumption 1 (i) For each $y$, there is a unique Ito process $\left\{Y_{t}\right\}_{t \geq 0}$ satisfying the above stochastic differential equation. ${ }^{9}$ (ii) The expectation $E\left[\int_{0}^{\infty} e^{-r t} Y_{t} d t\right]$ is finite for $r>0$.

A consumption plan $C=\left\{C_{t}\right\}_{t \geq 0}$ is a nonnegative process such that the present value is finite,

$$
E\left[\int_{0}^{\infty} e^{-r t} C_{t} d t\right]<\infty
$$

The agent derives utility from a consumption plan $C$ according to

$$
U_{0}^{a}(C) \equiv E\left[\int_{0}^{\infty} e^{-\rho t} u\left(C_{t}\right) d t\right]
$$

where $\rho>0$ is the subjective discount rate and $u: \mathbb{R}_{+} \rightarrow \mathbb{R}$. His continuation utility at date $t$ is given by

$$
U_{t}^{a}(C) \equiv E_{t}\left[\int_{t}^{\infty} e^{-\rho(s-t)} u\left(C_{s}\right) d s\right]
$$

Assume that $u$ satisfies

Assumption $2 u^{\prime}>0, u^{\prime \prime}<0, \lim _{c \downarrow 0} u^{\prime}(c)=\infty$ and $\lim _{c \uparrow \infty} u^{\prime}(c)=0$.

\footnotetext{
${ }^{9}$ Sufficient conditions are Lipschitz and growth conditions on $\mu$ and $\sigma$ : there is a constant $k$ such that for any $x$ and $y$ in $\mathbb{R}$,

$$
\begin{array}{ll}
|\mu(x)-\mu(y)| \leq k|x-y|, & |\mu(y)| \leq k\left(1+y^{2}\right), \\
|\sigma(x)-\sigma(y)| \leq k|x-y|, & |\sigma(y)| \leq k\left(1+y^{2}\right) .
\end{array}
$$
}

See Duffie (1996). 
By this assumption, there exists a strictly decreasing and continuously differentiable inverse function, $I: \mathbb{R}_{++} \rightarrow \mathbb{R}_{++}$, defined as $I(x)=\left(u^{\prime}\right)^{-1}(x)$, for all $x>0$. Define $I(0)=$ $\lim _{x \downarrow 0} I(x)=\infty$ and $I(\infty)=\lim _{x \uparrow \infty} I(x)=0$.

The agent does not have access to financial markets. To insure himself again income risk, he writes a contract with the risk-neutral principal. The agent hands in his endowment $Y$ to the principal, who then returns consumption $C$ to the agent. The principal can freely access financial markets and derives utility according to

$$
U^{p}(y, C) \equiv E\left[\int_{0}^{\infty} e^{-r t}\left(Y_{t}-C_{t}\right) d t\right] .
$$

Note that we allow $\rho \neq r$ in the model because when we interpret the principal as a financial intermediary, his discount rate $r$ is the interest rate. In a general equilibrium model, the endogenously determined interest rate is typically lower than the agent's subjective discount rate $\rho$ (see, e.g., Alvarez and Jermann (2000) and Krueger and Perri (2011)).

The key assumption of the model is that the agent has limited commitment. He can walk away from the contract and take an outside value at any time after signing the contract. Suppose that the outside value is given by $U_{d}\left(Y_{t}\right)$ at time $t$, where $U_{d}: \mathbb{R}_{+} \rightarrow \mathbb{R}$ is a measurable function. One example is that the outside value is equal to the autarky value so that

$$
U_{d}\left(Y_{t}\right)=E_{t}\left[\int_{t}^{\infty} e^{-\rho(s-t)} u\left(Y_{s}\right) d s\right]
$$

To ensure that the agent does not walk away, we impose the following participation constraint:

$$
U_{t}^{a}(C) \geq U_{d}\left(Y_{t}\right), \quad \forall t \geq 0
$$

In addition, we also impose the following initial individual rationality constraint or the promisekeeping constraint:

$$
U_{0}^{a}(C)=w,
$$

where $w$ is an initial promised value to the agent. We call a consumption plan enforceable if it satisfies (5) and (6). Let $\Gamma(y, w)$ denote the set of all enforceable consumption plans. By (5), we must assume that $w \geq U_{d}\left(Y_{0}\right)$ throughout the analysis.

We can now state the contracting problem as follows:

\section{Primal problem (one-sided limited commitment):}

$$
V(y, w)=\sup _{C \in \Gamma(y, w)} U^{p}(y, C) .
$$


We call this problem the primal problem and call $V$ the primal value function. The standard approach to solving this problem is to apply dynamic programming and use the agent's continuation value as a state variable (e.g., DeMarzo and Sannikov (2006), Sannikov (2008), Williams $(2009,2011)$ and Grochulski and Zhang (2011)). Let $W_{t} \equiv U_{t}^{a}(C)$ denote this state variable. By the Martingale Representation Theorem, there is a process $\left\{\sigma_{t}^{W}\right\}_{t \geq 0}$ such that $\left\{W_{t}\right\}_{t \geq 0}$ satisfies the following stochastic different equation:

$$
d W_{t}=\left(\rho W_{t}-u\left(C_{t}\right)\right) d t+\sigma_{t}^{W} d B_{t}
$$

The primal value function $V$ satisfies the following Hamilton-Jacobi-Bellman (HJB) equation:

$$
\begin{aligned}
r V\left(Y_{t}, W_{t}\right)= & \sup _{C_{t}, \sigma_{t}^{W}} Y_{t}-C_{t}+V_{y}\left(Y_{t}, W_{t}\right) \mu\left(Y_{t}\right)+\frac{1}{2} V_{y y}\left(Y_{t}, W_{t}\right) \sigma^{2}\left(Y_{t}\right) \\
& +V_{w}\left(Y_{t}, W_{t}\right)\left(\rho W_{t}-u\left(C_{t}\right)\right)+\frac{1}{2} V_{w w}\left(Y_{t}, W_{t}\right)\left(\sigma_{t}^{W}\right)^{2}+V_{y w}\left(Y_{t}, W_{t}\right) \sigma_{t}^{W} \sigma\left(Y_{t}\right),
\end{aligned}
$$

subject to (8) and the participation constraint, $W_{t} \geq U_{d}\left(Y_{t}\right) \cdot{ }^{10}$

After optimizing with respect to $C_{t}$ and $\sigma_{t}^{W}$, the HJB equation reduces to a nonlinear partial differential equation (PDE). Together with the participation constraint, the HJB equation is difficult to solve both analytically and numerically.

\subsection{First-best Allocation}

Before solving the problem with limited commitment, we first present the solution to the firstbest benchmark in which the participation constraint (5) is removed. We make the following assumption:

Assumption 3 The integral $\int_{0}^{\infty} e^{-\rho t} u\left(I\left(e^{(\rho-r) t}\right)\right) d t$ is finite. ${ }^{11}$ The initial promised value $w$ satisfies

$$
\lim _{\phi \downarrow 0} \int_{0}^{\infty} e^{-\rho t} u\left(I\left(e^{(\rho-r) t} / \phi\right)\right) d t=\frac{u(0)}{\rho}<w<\frac{u(\infty)}{\rho}=\lim _{\phi \uparrow \infty} \int_{0}^{\infty} e^{-\rho t} u\left(I\left(e^{(\rho-r) t} / \phi\right)\right) d t .
$$

By this assumption and Assumption 2, there exists a unique Lagrange multiplier $\phi^{*}>$ 0 associated with the promise-keeping constraint (6) such that the first-best consumption is deterministic and is given by

$$
C_{t}^{F B}=I\left(e^{(\rho-r) t} / \phi^{*}\right), \text { for all } t \geq 0 .
$$

\footnotetext{
${ }^{10}$ In models with two-sided limited commitment, there is also an endogenous upper bound on $W_{t}$. See the end of Section 3.2 for a discussion and the examples in Section 3.3 and Appendix C.

${ }^{11}$ This assumption implies that $\int_{0}^{\infty} e^{-\rho t} u\left(I\left(e^{(\rho-r) t} / \phi\right)\right) d t$ is finite for each $\phi>0$.
} 
For instance, if $u(c)=c^{\alpha} / \alpha, 0 \neq \alpha<1$, then

$$
C_{t}^{F B}=\phi^{* \frac{1}{1-\alpha}} e^{\frac{r-\rho}{1-\alpha} t}, \text { where } \phi^{*}=\left[\frac{\alpha w(\rho-\alpha r)}{1-\alpha}\right]^{\frac{1-\alpha}{\alpha}} .
$$

Assumption 3 is satisfied if and only if $\rho>\alpha r$ and $\alpha w>0$. In the first best, the risk-neutral principal bears all uncertainty and fully insures the risk-averse agent. In particular, if $\rho=r$, then the agent's first-best consumption plan is constant over time. If $r>(<) \rho$, the agent is more (less) patient than the principal so that the agent's first-best consumption increases (decreases) over time.

\subsection{Heuristic Derivation of the Dual Problem}

In this subsection, we use informal heuristic arguments to derive the dual problem by ignoring some technical issues. We will provide formal results in the next two subsections, with rigorous proofs given in the appendix. First, similar to the Lagrange method in discrete time (e.g., Marcet and Marimon (1998), Ljungqvist and Sargent (2004), and Messner, Pavoni, and Sleet (2011, 2012)), we write down the Lagrangian in continuous time

$$
\begin{aligned}
\mathfrak{L}= & E\left[\int_{0}^{\infty} e^{-r t}\left(Y_{t}-C_{t}\right) d t\right]+\phi\left(E\left[\int_{0}^{\infty} e^{-\rho s} u\left(C_{s}\right) d s\right]-w\right) \\
& +E\left[\int_{0}^{\infty} e^{-r t} \lambda_{t}\left(\int_{t}^{\infty} e^{-\rho(s-t)} u\left(C_{s}\right) d s-U_{d}\left(Y_{t}\right)\right) d t\right]
\end{aligned}
$$

where $e^{-r t} \lambda_{t} \geq 0$ is the Lagrange multiplier associated with the participation constraint (5) at each time $t \geq 0$ and $\phi>0$ is the Lagrange multiplier associated with the promise-keeping constraint (6). It must be the case that $\phi>0$ because raising the agent's promised value would increase the agent's consumption and reduce the principal's value.

Using integration by parts, we can compute that ${ }^{12}$

$$
E\left[\int_{0}^{\infty} e^{-r t} \lambda_{t}\left(\int_{t}^{\infty} e^{-\rho(s-t)} u\left(C_{s}\right) d s\right) d t\right]=E\left[\int_{0}^{\infty}\left(\int_{0}^{t} e^{(\rho-r) s} \lambda_{s} d s\right) e^{-\rho t} u\left(C_{t}\right) d t\right] .
$$

\footnotetext{
${ }^{12}$ Specifically,$$
E\left[\int_{0}^{\infty} e^{-r t} \lambda_{t}\left(\int_{t}^{\infty} e^{-\rho(s-t)} u\left(C_{s}\right) d s\right) d t\right]=E\left[\int_{0}^{\infty}\left(\int_{t}^{\infty} e^{-\rho s} u\left(C_{s}\right) d s\right) d\left(\int_{0}^{t} e^{(\rho-r) s} \lambda_{s} d s\right)\right]
$$$$
=\left.E\left[\left(\int_{t}^{\infty} e^{-\rho s} u\left(C_{s}\right) d s\right)\left(\int_{0}^{t} e^{(\rho-r) s} \lambda_{s} d s\right)\right]\right|_{0} ^{\infty}
$$$$
-E\left[\int_{0}^{\infty}\left(\int_{0}^{t} e^{(\rho-r) s} \lambda_{s} d s\right) d\left(\int_{t}^{\infty} e^{-\rho s} u\left(C_{s}\right) d s\right)\right]
$$$$
=E\left[\int_{0}^{\infty}\left(\int_{0}^{t} e^{(\rho-r) s} \lambda_{s} d s\right) e^{-\rho t} u\left(C_{t}\right) d t\right] \text {. }
$$ 
Plugging this equation into the Lagrangian, we obtain

$$
\begin{aligned}
\mathfrak{L}= & E\left[\int_{0}^{\infty} e^{-r t}\left(Y_{t}-C_{t}\right) d t\right]-E\left[\int_{0}^{\infty} e^{-r t} \lambda_{t} U_{d}\left(Y_{t}\right) d t\right] \\
& +E\left[\int_{0}^{\infty}\left(\int_{0}^{t} e^{(\rho-r) s} \lambda_{s} d s+\phi\right) e^{-\rho t} u\left(C_{t}\right) d t\right]-\phi w .
\end{aligned}
$$

As in Marcet and Marimon (1998), we define a costate process $X$ as the cumulative amounts of the Lagrangian multipliers,

$$
X_{t} \equiv \int_{0}^{t} e^{(\rho-r) s} \lambda_{s} d s+\phi, \quad t \geq 0
$$

This process is increasing, continuous, and satisfies

$$
d X_{t}=e^{(\rho-r) t} \lambda_{t} d t
$$

Using this process, the Lagrangian becomes

$$
\begin{aligned}
\mathfrak{L}= & E\left[\int_{0}^{\infty} e^{-r t} Y_{t} d t\right]-E\left[\int_{0}^{\infty} e^{-\rho t} U_{d}\left(Y_{t}\right) d X_{t}\right] \\
& +E\left[\int_{0}^{\infty} e^{-r t}\left(X_{t} e^{-(\rho-r) t} u\left(C_{t}\right)-C_{t}\right) d t\right]-X_{0} w .
\end{aligned}
$$

To derive the dual problem, we first choose consumption to maximize $\mathfrak{L}$. Define the dual function of $u$ as $^{13}$

$$
\tilde{u}(z) \equiv \max _{c>0}\{z u(c)-c\}, \text { for } z>0 .
$$

Since $u$ is strictly concave, the solution is $c^{*}=I(1 / z)$. We can show that $I(1 / z)$ is strictly increasing in $z$ and $\tilde{u}(z)$ is strictly convex in $z{ }^{14}$ Optimizing over $C_{t}$ in (11) yields

$$
\mathcal{L}(X) \equiv E\left[\int_{0}^{\infty} e^{-r t}\left(Y_{t}+\tilde{u}\left(X_{t} e^{-(\rho-r) t}\right)\right) d t\right]-E\left[\int_{0}^{\infty} e^{-\rho t} U_{d}\left(Y_{t}\right) d X_{t}\right]-X_{0} w
$$

We then choose the process $X$ to minimize $\mathcal{L}(X)$.

\footnotetext{
${ }^{13}$ Note that this dual function is not the same as the following convex conjugate function often defined in the literature:$$
\tilde{u}(y)=\sup _{x>0} u(x)-x y, \quad y>0 .
$$

${ }^{14}$ This result follows from

$$
\frac{d I(1 / z)}{d z}=\frac{-1}{z^{2} u^{\prime \prime}(I(1 / z))}>0
$$

and the fact that $\tilde{u}^{\prime}(z)=u(I(1 / z))$ increases in $z$.
} 


\section{Dual problem (one-sided limited commitment):}

$$
\inf _{X \in \mathcal{I}} \mathcal{L}(X)
$$

where $\mathcal{I}$ denotes the set of all increasing, right continuous processes $X$ with left limits and starting at positive initial values such that

$$
\begin{aligned}
& E\left[\int_{0}^{\infty} e^{-\rho t}\left|U_{d}\left(Y_{t}\right)\right| d X_{t}\right]<\infty, \\
& E\left[\int_{0}^{\infty} e^{-r t}\left|\tilde{u}\left(X_{t} e^{-(\rho-r) t}\right)\right| d t\right]<\infty .
\end{aligned}
$$

Note that in this formulation of the dual problem, the set $\mathcal{I}$ of feasible processes contains all increasing and right continuous processes with left limits. A process $X \in \mathcal{I}$ is generally not absolutely continuous with respect to time $t$. Thus, equations (9) and (10) will not hold in a rigorous mathematical sense. Our previous derivation is purely heuristic and will not be used in our formal proofs. ${ }^{15}$ But without using a heuristic derivation, it is far from routine to formulate the dual problem. We also emphasize that the infimum in (14) is taken with respect to the whole sample path $\left\{X_{t}\right\}_{t \geq 0}$, including the initial value $X_{0}>0$. Finally, the integrability conditions in (15) and (16) ensure that $\mathcal{L}(X)$ is finite.

\subsection{Weak and Strong Duality}

We break up the dual problem (14) into two sub-problems. First, define

$$
L(y, x, X) \equiv E\left[\int_{0}^{\infty} e^{-r t}\left(Y_{t}+\tilde{u}\left(X_{t} e^{-(\rho-r) t}\right)\right) d t\right]-E\left[\int_{0}^{\infty} e^{-\rho t} U_{d}\left(Y_{t}\right) d X_{t}\right]
$$

where the expectations are conditional on $X_{0}=x$ and $Y_{0}=y$. Define the dual value function as

$$
\tilde{V}(y, x) \equiv \inf _{X \in \mathcal{I}(x)} L(y, x, X) \quad \text { for any } x>0
$$

where $\mathcal{I}(x)$ denotes the set of all processes in $\mathcal{I}$ starting at $x>0$. Second, we study the problem:

$$
\inf _{x>0} \tilde{V}(y, x)-x w
$$

The following property is useful.

Proposition $1 \tilde{V}(y, x)$ is convex in $x$.

Now, we study the relationship between the primal problem (7) and the dual problem (14).

\footnotetext{
${ }^{15}$ In fact, we will show later that the optimal $X$ is a regulated Brownian motion which is not absolutely continuous (Harrison (1985)).
} 
Theorem 1 (weak duality) For every enforceable plan $C \in \Gamma(y, w)$, every $x>0$, and every $X \in \mathcal{I}(x)$, the following inequality holds:

$$
U^{p}(y, C) \leq L(y, x, X)-x w
$$

Equality holds if and only if for all $t \geq 0$,

$$
\begin{aligned}
X_{t} e^{-(\rho-r) t} u^{\prime}\left(C_{t}\right)-1 & =0 \\
\int_{0}^{t} e^{-\rho s}\left(U_{s}^{a}(C)-U_{d}\left(Y_{s}\right)\right) d X_{s} & =0 .
\end{aligned}
$$

This theorem shows that the objective function $L(y, x, X)-x w$ in the dual problem provides an upper bound on the objective function $U^{p}(y, C)$ in the primal problem. An immediate corollary is that the primal value function is weakly below the dual value function:

$$
V(y, w) \leq \inf _{x>0} \tilde{V}(y, x)-x w .
$$

This result is called weak duality.

Equations (21)-(22) give conditions under which equality in (20) holds. These conditions are analogous to the Kuhn-Tucker conditions in the discrete-time model analyzed in Marcet and Marimon (1998), Ljungqvist and Sargent (2004), and Zhang (2013). In particular, equation (21) is the first-order condition for consumption, and equation (22) is a continuous-time version of the complementary slackness condition for optimality. The latter equation is related to the Skorokhod problem and shows that $X_{t}$ increases only when the participation constraint $U_{t}^{a}(C) \geq U_{d}\left(Y_{t}\right)$ binds.

The following theorem shows that a solution to the dual problem implies a solution to the primal problem and hence the equalities in (20) and (23) hold.

Theorem 2 (strong duality) Suppose that $X^{*} \in \mathcal{I}$ is a solution to the dual problem (14). Let

$$
C_{t}^{*} \equiv I\left(e^{(\rho-r) t} / X_{t}^{*}\right), \quad t \geq 0 .
$$

If $C^{*}$ satisfies condition (1) and if condition (16) holds for the processes $X^{\delta}=X^{*}+\delta$ and $\bar{X}^{ \pm \delta}=X^{*}(1 \pm \delta)$ for some small $\delta>0$, then $C^{*}$ is a solution to the primal problem (7). In addition,

$$
V(y, w)=\inf _{x>0} \tilde{V}(y, x)-x w .
$$

The idea of the proof of this theorem is to first show that $C^{*}$ is enforceable and then show that $C^{*}$ and $X^{*}$ satisfy (21)-(22). As a result, we can apply Theorem $1 .{ }^{16}$ To make this argument

\footnotetext{
${ }^{16}$ Theorems 1 and 2 are analogous to Propositions 11-13 for the discrete-time setting in Messner, Pavoni, and Sleet (2011).
} 
work, we use perturbation around $X^{*}$. The integrability conditions in the theorem ensure that certain functions are integrable after small perturbations. These are simple sufficient conditions used when we take limits in the proof. They can be easily verified in our examples presented later.

Theorem 2 shows that after solving the dual problem, optimal consumption in the primal problem can be completely characterized by the function $I\left(e^{(\rho-r) t} / X_{t}^{*}\right)$. By the previous analysis, this function is strictly increasing with $e^{(r-\rho) t} X_{t}^{*}$. By $(21)$, this term is the ratio of the marginal utilities of the principal and the agent. This result can be generalized to the case of a risk-averse principal and to the case of two-sided limited commitment, as will be shown in Section 3. Alternatively, we can interpret $e^{(r-\rho) t} X_{t}^{*}$ as the "temporary relative Pareto weight" on the principal and the agent, as in Chapter 20 of Ljungqvist and Sargent (2004). In the next subsection, we provide a dynamic programming characterization of the dual problem.

\subsection{Dynamic Programming}

Since the exogenous state process $Y$ in our model is assumed to be Markovian, we can provide a dynamic programming characterization for the dual problem. We adopt the ratio of the marginal utilities of the principal and the agent as a state variable. This ratio is equal to the discount-rate-adjusted costate variable $Z_{t} \equiv e^{-(\rho-r) t} X_{t}$ and satisfies the dynamics:

$$
d Z_{t}=Z_{t} / X_{t} d X_{t}-(\rho-r) Z_{t} d t, \quad X_{0}=Z_{0}=z>0 .
$$

We then rewrite the problem (18) as

$$
J(y, z) \equiv \inf _{X \in \mathcal{I}(z)} E\left[\int_{0}^{\infty} e^{-r t}\left(Y_{t}+\tilde{u}\left(Z_{t}\right)\right) d t\right]-E \int_{0}^{\infty} e^{-\rho t} U_{d}\left(Y_{t}\right) d X_{t}
$$

subject to (24). This is a singular control or instantaneous control problem in control theory (e.g., Harrison and Taksar (1983), Fleming and Soner (2006), or Stokey (2008)), where $X$ is the control process and $Y$ and $Z$ are state processes. Note that $J$ and $\tilde{V}$ are related by

$$
J\left(Y_{0}, Z_{0}\right)=\tilde{V}\left(Y_{0}, X_{0}\right)
$$

By standard theory, the HJB equation is formulated in terms of a variational inequality

$$
\min \left\{y+\tilde{u}(z)+\mathcal{A} J(y, z), J_{z}(y, z)-U_{d}(y)\right\}=0, \quad(y, z) \in \mathbb{R}_{+} \times \mathbb{R}_{++},
$$

where

$$
\mathcal{A} J(y, z)=(r-\rho) z J_{z}(y, z)+J_{y}(y, z) \mu(y)+\frac{1}{2} J_{y y}(y, z) \sigma^{2}(y)-r J(y, z) .
$$


The variational inequality (26) partitions the state space into two regions:

$$
\begin{aligned}
& \Omega_{1}=\left\{(y, z) \in \mathbb{R}_{+} \times \mathbb{R}_{++}: J_{z}(y, z)=U_{d}(y)\right\} \\
& \Omega_{2}=\left\{(y, z) \in \mathbb{R}_{+} \times \mathbb{R}_{++}: J_{z}(y, z)>U_{d}(y)\right\} .
\end{aligned}
$$

Since $J(y, z)$ is convex in $z$ by Proposition 1 , a free boundary $z=\varphi(y)$ defined by

$$
\varphi(y)=\inf \left\{z^{\prime}>0: J_{z}\left(y, z^{\prime}\right)>U_{d}(y)\right\}
$$

separates $\Omega_{1}$ and $\Omega_{2}$. See Figure 1 in Section 2.5 for an illustration.

If initially $\left(Y_{0}, Z_{0}\right) \in \Omega_{1}$, then $X$ should jump up immediately, such that $Z$ reaches the boundary. On the other hand, if $(y, z) \in \Omega_{2}$, then

$$
y+\tilde{u}(z)+\mathcal{A} J(y, z)=0,
$$

and $X$ must stay constant. Thus, we call $\Omega_{1}$ and $\Omega_{2}$ the jump and the no-jump regions, respectively. If $\left(Y_{0}, Z_{0}\right)$ starts inside the no-jump region, then $X$ will be a process that regulates $Z$ so that $\left(Y_{t}, Z_{t}\right)$ stays inside the no-jump region. The sample path of $X$ at the optimum must have the property that it increases only when $\left(Y_{t}, Z_{t}\right)$ hits the free boundary, at which time the participation constraints bind.

Following the standard dynamic programming theory, we state a verification theorem.

Theorem 3 (verification) Let $J(y, z)$ be a twice continuously differentiable solution to (26) such that for any $Z$ in (24) and $X \in \mathcal{I}(z)$, (i) the process defined by

$$
\int_{0}^{t} e^{-r s} J_{y}\left(Y_{s}, Z_{s}\right) \sigma\left(Y_{s}\right) d B_{s}, \quad t \geq 0
$$

is a martingale, and (ii)

$$
\lim _{t \rightarrow \infty} E\left[e^{-r t} J\left(Y_{t}, Z_{t}\right)\right]=0
$$

Suppose further that $Z_{t}^{*}=e^{-(\rho-r) t} X_{t}^{*}$, where $X^{*} \in \mathcal{I}(z)$ and $(y, z) \in \Omega_{2}$, is such that (i)

$$
Y_{t}+\tilde{u}\left(Z_{t}^{*}\right)+\mathcal{A} J\left(Y_{t}, Z_{t}^{*}\right)=0
$$

for all $t \geq 0$, (ii) for all $t \geq 0$,

$$
\int_{0}^{t} e^{-\rho s}\left(J_{z}\left(Y_{s}, Z_{s}^{*}\right)-U_{d}\left(Y_{s}\right)\right) d X_{s}^{*}=0 .
$$

Then $X^{*}$ is the optimal solution to problem (25) and $J$ is the associated dual value function. Suppose further that $J(y, z)$ is strictly convex in $z$ on $\Omega_{2}$, there exists $z^{*}>0$ such that 
$J_{z}\left(y, z^{*}\right)=w$, and the conditions in Theorem 2 hold. Then $X_{0}^{*}=z^{*}$ is the optimal solution to problem (19) and the primal value function is given by $V(y, w)=J\left(y, z^{*}\right)-z^{*} w$. The optimal consumption plan, continuation values, and the marginal utility ratio are, respectively, given by

$$
C_{t}^{*}=I\left(1 / Z_{t}^{*}\right), \quad W_{t}^{*}=J_{z}\left(Y_{t}, Z_{t}^{*}\right), \quad Z_{t}^{*}=-V_{w}\left(Y_{t}, W_{t}^{*}\right) .
$$

Equation (30) is a linear PDE, which is easier to solve explicitly, as illustrated in Section 2.5. Condition (28) is a technical condition used to verify the optimality of $X^{*}$ by the martingale method. Condition (29) is the transversality condition that usually appears in infinite-horizon control problems. Condition (31) indicates that $X^{*}$ increases if and only if $J_{z}\left(Y_{t}, Z_{t}^{*}\right)=U_{d}\left(Y_{t}\right)$. It is also related to (22) and may be interpreted as a complementary slackness condition associated with the participation constraints. The solution $X^{*}$ is related to the classical Skorokhod problem. As is well known (e.g., Harrison and Taksar (1983)), we can express $X^{*}$ as

$$
X_{t}^{*}=\max \left\{z^{*}, \max _{s \in[0, t]} \varphi\left(Y_{s}\right) e^{(\rho-r) s}\right\} .
$$

In addition, $X^{*}$ also admits a local time characterization, which we will not pursue here.

Equation (32) shows that optimal consumption can be completely characterized by $Z_{t}^{*}$, the ratio of the marginal utilities of the principal and the agent, which, by the Envelope Theorem, is equal to the negative slope of the Pareto frontier given an income level. Equation (32) also shows that the agent's continuation value is equal to the partial derivative of the dual value function with respect to the marginal utility ratio, $Z_{t}^{*}$, given an income level. Thus, optimal consumption can be expressed as a function of the income level and the agent's continuation value, as in the literature (e.g., Ljungqvist and Sargent (2004)).

Since $J_{z}(y, \varphi(y))=U_{d}(y)$ on the free boundary, it follows from $w \geq U_{d}\left(Y_{0}\right)$ that

$$
J_{z}\left(Y_{0}, Z_{0}^{*}\right)=w \geq J_{z}\left(Y_{0}, \varphi\left(Y_{0}\right)\right) .
$$

When $J$ is strictly convex in $z$ on $\Omega_{2}$, we deduce that $X_{0}^{*}=Z_{0}^{*} \geq \varphi\left(Y_{0}\right)$, implying that the optimal starting value of $X^{*}$ or $Z^{*}$ is inside the no-jump region. Thus, there is no jump in $X^{*}$ or $Z^{*}$ and both processes are continuous.

\subsection{Example I}

We now introduce the participation constraint (5) to the example studied in Section 2.1. In this case, the first-best allocation cannot be achieved. Thus, the agent must also bear income uncertainty. To derive a closed-form solution, we assume that the agent's income $Y$ follows a geometric Brownian motion

$$
d Y_{t}=\mu Y_{t} d t+\sigma Y_{t} d B_{t}, \quad Y_{0}=y>0
$$


where $\sigma>0$. We assume $r>\mu+\sigma^{2} / 2$ so that the present value of income discounted at $r$ is finite. This assumption also allows us to check condition (28) in Theorem $3{ }^{17}$

Let $u(c)=c^{\alpha} / \alpha$ for $0 \neq \alpha<1$. The $\log$ utility case corresponds to $\alpha=0$. We assume $\rho>\alpha r$ so that the first-best allocation exists. Suppose that the agent's outside option is autarky so that

$$
U_{d}(y)=E\left[\int_{0}^{\infty} e^{-\rho t} u\left(Y_{t}\right) d t \mid Y_{0}=y\right]=\kappa y^{\alpha}
$$

where we define

$$
\kappa \equiv \frac{1}{\alpha\left(\rho-\alpha \mu-\alpha(\alpha-1) \sigma^{2} / 2\right)},
$$

and assume that $\rho>\alpha \mu+\alpha(\alpha-1) \sigma^{2} / 2$ to ensure a finite autarky value.

\subsubsection{Solution}

We can derive that

$$
\tilde{u}(z)=\frac{1-\alpha}{\alpha} z^{\frac{1}{1-\alpha}}, \text { for } z>0,
$$

and the optimal consumption rule is $c^{*}=z^{\frac{1}{1-\alpha}}$. In the no-jump region, equation (30) becomes

$$
r J(y, z)=y+\frac{1-\alpha}{\alpha} z^{\frac{1}{1-\alpha}}+(r-\rho) z J_{z}(y, z)+J_{y}(y, z) \mu y+\frac{\sigma^{2}}{2} y^{2} J_{y y}(y, z) .
$$

This is a linear PDE. Given the two free-boundary conditions, $J_{z}(y, z)=U_{d}(y)$ and $J_{z z}(y, z)=$ 0 (often called the value-matching and super-contact conditions in the literature, e.g., Dumas (1991)), we can derive the following general solution: ${ }^{18}$

$$
J(y, z)=\frac{y}{r-\mu}+\frac{(1-\alpha)^{2}}{(\rho-\alpha r) \alpha} z^{\frac{1}{1-\alpha}}+A z^{\frac{1-\beta}{1-\alpha}} y^{\beta},
$$

where $A$ is a constant to be determined and $\beta$ is the positive root of the characteristic equation

$$
r=(r-\rho) \frac{1-\beta}{1-\alpha}+\mu \beta+\frac{\sigma^{2}}{2} \beta(\beta-1) .
$$

We can also show that $\beta>1$. We rule out the particular solution corresponding to the negative root because this solution makes $J(y, z)$ converge to infinity as $y \downarrow 0$. But $J(y, z)$ should converge to the finite first-best value since the autarky value is so small that the participation constraints will not bind when $y \downarrow 0$.

\footnotetext{
${ }^{17}$ We will provide additional proofs for this example in Appendix B.

${ }^{18}$ For $\log$ utility, the dual value function is given by

$$
J(y, z)=\frac{y}{r-\mu}+\frac{z \ln (z)}{\rho}+\frac{r-2 \rho}{\rho^{2}} z+A z^{1-\beta} y^{\beta},
$$

where $A=\frac{b^{\beta}}{\rho(1-\beta) \beta}$ and $b=e^{\left(\mu-\sigma^{2} / 2-r\right) \rho^{-1}-\beta^{-1}+1}$. The free boundary and consumption rule are the limits when $\alpha \rightarrow 0$.
} 
From (37) and the above two free-boundary conditions, we can derive that

$$
\begin{aligned}
\frac{1-\alpha}{(\rho-r \alpha) \alpha} z^{\frac{\alpha}{1-\alpha}}+A \frac{1-\beta}{1-\alpha} z^{\frac{\alpha-\beta}{1-\alpha}} y^{\beta} & =\kappa y^{\alpha}, \\
\frac{1}{(\rho-r \alpha)} z^{\frac{\alpha}{1-\alpha}-1}+A \frac{1-\beta}{1-\alpha} \frac{\alpha-\beta}{1-\alpha} z^{\frac{\alpha-\beta}{1-\alpha}-1} y^{\beta} & =0 .
\end{aligned}
$$

Substituting the second equation above into the first one, we can derive the free-boundary, $z=b y^{1-\alpha}$, where

$$
b=\left[\frac{\alpha(\rho-\alpha r)(\beta-\alpha) \kappa}{\beta(1-\alpha)}\right]^{\frac{1-\alpha}{\alpha}}>0 .
$$

Here, the sign can be verified using the definition of $\kappa$ and the assumptions on parameter values.

Substituting the free boundary $z=b y^{1-\alpha}$ into the super-contact condition $J_{z z}(y, z)=0$ or (39), we can derive

$$
A=-\frac{(1-\alpha)^{2} b^{\frac{\beta}{1-\alpha}}}{(1-\beta)(\alpha-\beta)(\rho-\alpha r)}<0 .
$$

We then obtain the no-jump region $\left\{(y, z) \in \mathbb{R}_{++}^{2}: z \geq b y^{1-\alpha}\right\}$ and the dual value function in this region given in (37). In the jump region $\left\{(y, z) \in \mathbb{R}_{++}^{2}: z<b y^{1-\alpha}\right\}$, we use $J_{z}(y, z)=$ $U_{d}(y)$ and $\lim _{z \downarrow b y^{1-\alpha}} J(y, z)=\lim _{z \uparrow b y^{1-\alpha}} J(y, z)$ to derive that

$$
J(y, z)=\left(z-b y^{1-\alpha}\right) U_{d}(y)+J\left(b y^{1-\alpha}, y\right), \quad \text { for } z<b y^{1-\alpha} .
$$

In Appendix B, we show that $J(y, z)$ is strictly convex in $z$ in the no-jump region. Using equation, $J_{z}(y, z)=w$, or

$$
\frac{1-\alpha}{(\rho-r \alpha) \alpha} z^{\frac{\alpha}{1-\alpha}}+A \frac{1-\beta}{1-\alpha} z^{\frac{\alpha-\beta}{1-\alpha}} y^{\beta}=w
$$

we can derive a unique solution for $z$ when $w \in\left[U_{d}(y), \infty\right)$ for $\alpha>0$ and $w \in\left[U_{d}(y), 0\right)$ for $\alpha<0$. This solution is used as the initial value for the processes $Z_{t}^{*}$ and $X_{t}^{*}$.

\subsubsection{Numerical Illustrations}

Figure 1 plots the state space. The curve $z=b y^{1-\alpha}$ partitions the state space into the jump and no-jump regions. Whenever the initial promised value $w \geq U_{d}(y)$, the initial state $(y, z)$ must lie in the no-jump region. The optimal $X^{*}$ ensures that $\left(Y_{t}, Z_{t}^{*}\right)$ will never leave the no-jump region. Whenever $Y_{t}$ is high enough and hits the free boundary, $X_{t}^{*}$ will rise instantaneously to make $\left(Y_{t}, Z_{t}^{*}\right)$ stay in the no-jump region.

Figure 2 plots the dual and primal value functions given three different values of $y$. The three dots on the left panel of this figure indicate the points $\left(y, b y^{1-\alpha}\right)$ on the free boundaries. The lowest promised value to the agent for each $y$ is determined by $w_{\min }(y)=J_{z}\left(y, b y^{1-\alpha}\right)=U_{d}(y)$, 


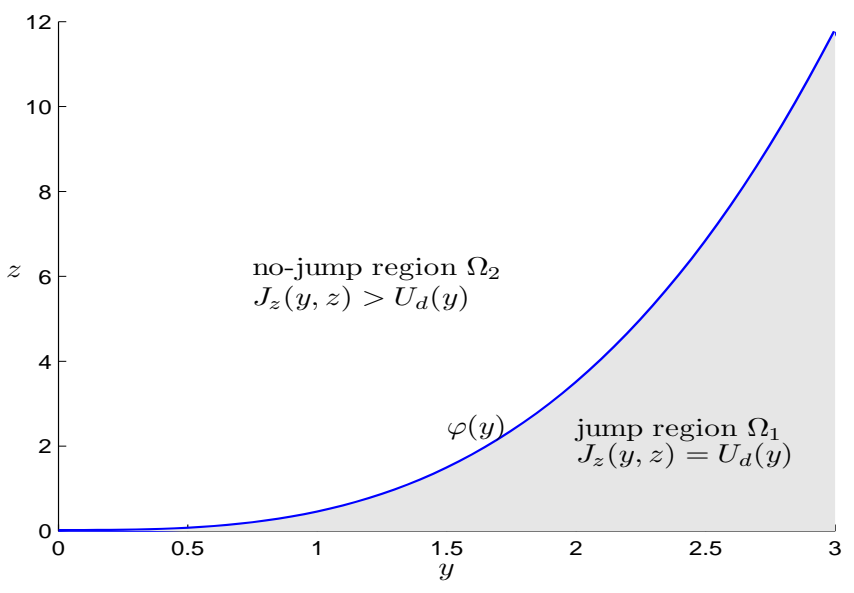

Figure 1: The state space for Example I. The curve $z=\varphi(y)=b y^{1-\alpha}$ partitions the state space into a jump region $\Omega_{1}$ and a no-jump region $\Omega_{2}$. Parameter values are given by $\mu=0.02$, $\sigma=0.1, \rho=0.04, r=0.04$, and $\alpha=-2$.
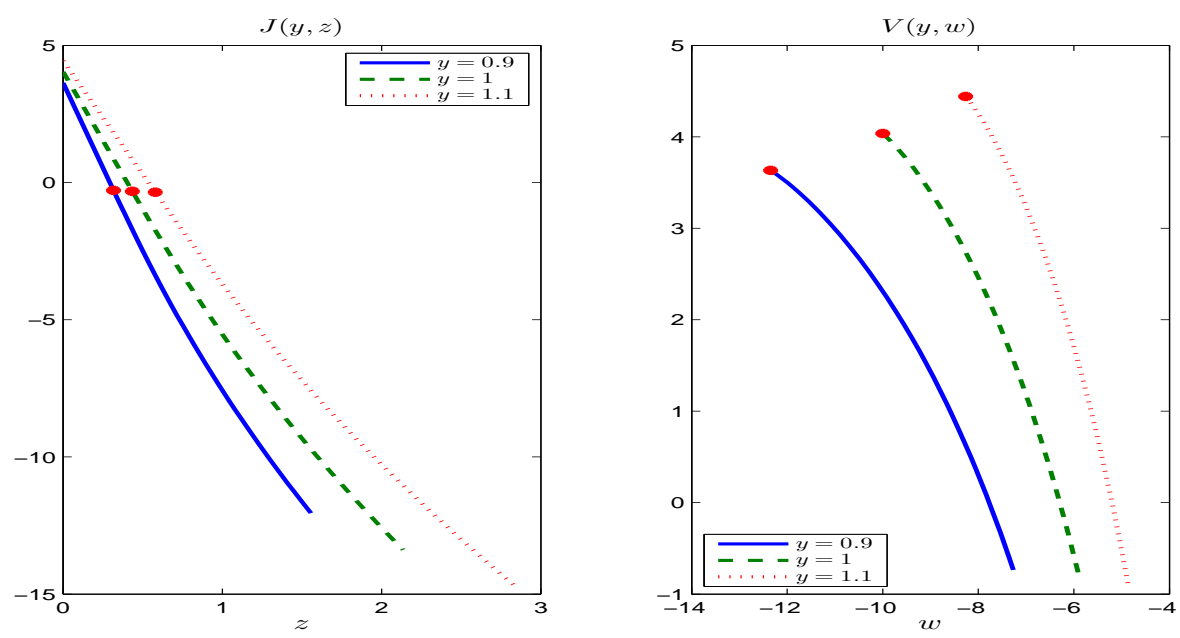

Figure 2: The dual and primal value functions for Example I. Parameter values are given by $\mu=0.02, \sigma=0.1, \rho=0.04, r=0.04$, and $\alpha=-2$. 

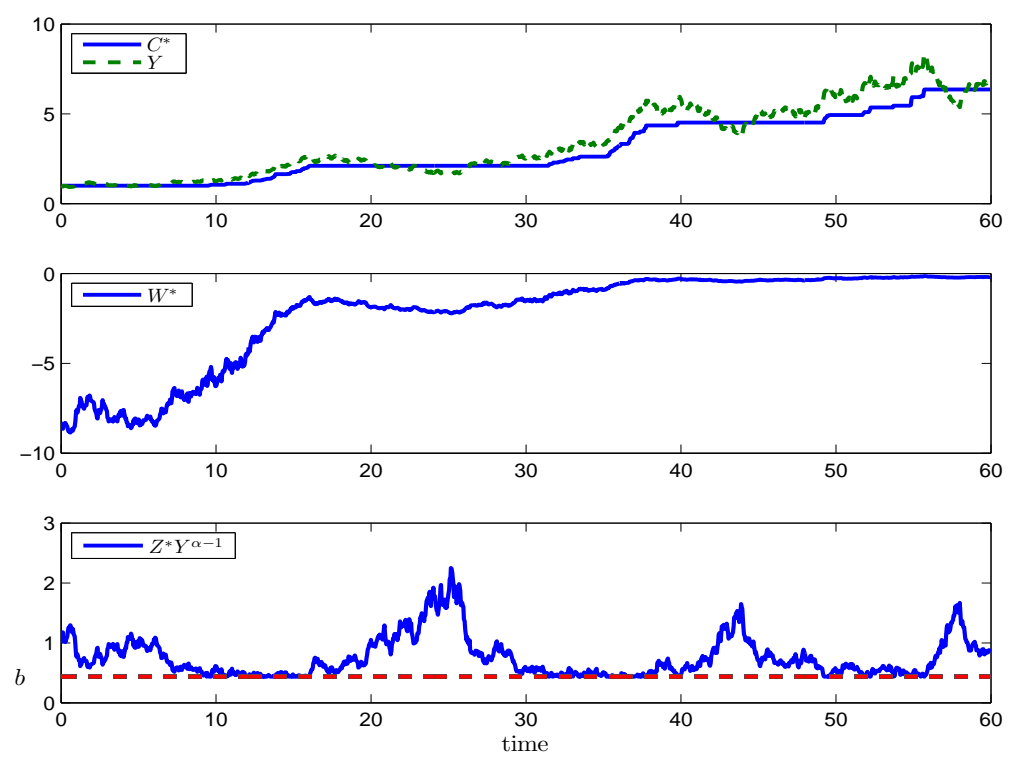

Figure 3: Simulated paths of consumption $C^{*}$, incomes $Y$, the agent's continuation values $W^{*}$, and the process $Z^{*} Y^{\alpha-1}$ for Example I. Parameter values are given by $\mu=0.02, \sigma=0.1$, $\rho=0.04, r=0.04$, and $\alpha=-2$.

which is indicated by the dots on the right panel. This panel shows the value function $V(y, w)$, which gives the Pareto frontier conditional on $y$. This frontier is concave and decreasing. Note that when $w$ is small, the principal makes positive profits, but when $w$ is sufficiently large, the principal incurs losses. This gives the principal an incentive to renege on the contract if he lacks commitment. In Section 3, we will analyze this case.

Figure 3 plots the simulated paths of incomes $Y_{t}$, optimal consumption $C_{t}^{*}$, and continuation values $W_{t}^{*}=J_{z}\left(Y_{t}, Z_{t}^{*}\right)$. The optimal consumption plan is given by $C_{t}^{*}=\left(Z_{t}^{*}\right)^{\frac{1}{1-\alpha}}$, where

$$
X_{t}^{*}=\max \left\{X_{0}^{*}, \max _{s \in[0, t]} b Y_{s}^{1-\alpha} e^{(\rho-r) s}\right\} \quad \text { and } \quad Z_{t}^{*}=e^{-(\rho-r) t} X_{t}^{*} .
$$

Since $X^{*}$ is an increasing process and rises whenever $Y_{t}$ is high enough to hit the free boundary, $C^{*}$ also follows this pattern if $\rho=r$.

\subsubsection{Stationary Distribution}

By Harrison (1985), the process $\ln \left(C_{t}^{*} / Y_{t}\right)$ is a regulated Brownian motion with drift $\sigma^{2} / 2-$

$\mu+(1-\alpha)^{-1}(r-\rho)$ and volatility $-\sigma$ on $\left[(1-\alpha)^{-1} \ln b, \infty\right)$. By Proposition 5.5 in Harrison (1985) or Proposition 10.8 in Stokey (2008), when $(1-\alpha)^{-1}(r-\rho)+\sigma^{2} / 2<\mu, \ln \left(C_{t}^{*} / Y_{t}\right)$ has 
a unique stationary distribution with the density function

$$
p(x)=\frac{-\delta e^{\delta x}}{b^{\frac{\delta}{1-\alpha}}}, \quad x \in\left[\frac{\ln b}{1-\alpha}, \infty\right),
$$

where $\delta \equiv\left(2(r-\rho) /(1-\alpha)+\sigma^{2}-2 \mu\right) / \sigma^{2}$. This is in sharp contrast to the discrete-time case analyzed by Ljungqvist and Sargent (2004), who show that the optimal consumption plan converges to the first best in the long run if the agent's endowment follows a finite-state IID process.

\section{Two-Sided Limited Commitment}

This section extends our methodology to the case of two-sided limited commitment. We extend the model in Section 2 in two respects. First, we allow the principal to be risk averse. That is, the principal derives utility according to

$$
U^{p}(y, C)=E\left[\int_{0}^{\infty} e^{-r t} u^{p}\left(A\left(Y_{t}\right)-C_{t}\right) d t\right]
$$

where $u^{p}: \mathbb{R}_{+} \rightarrow \mathbb{R}$ satisfies $\left(u^{p}\right)^{\prime}>0$ and $\left(u^{p}\right)^{\prime \prime} \leq 0$. When the principal is risk neutral $\left(\left(u^{p}\right)^{\prime \prime}=0\right)$, we allow him to access financial markets so that his consumption can be negative. Here, $A\left(Y_{t}\right)$ represents the aggregate endowment and $C_{t}$ is the agent's consumption, where $A: \mathbb{R}_{+} \rightarrow \mathbb{R}_{+}$is a continuous and increasing function. Define the principal's continuation value as

$$
U_{t}^{p}(A(Y)-C)=E_{t}\left[\int_{t}^{\infty} e^{-r(s-t)} u^{p}\left(A\left(Y_{s}\right)-C_{s}\right) d s\right] .
$$

For simplicity, we have assumed that the agent's endowment, $Y$, is the only exogenous state process, and that the principal's endowment is $A(Y)-Y$.

Second, we allow the principal to have limited commitment. He can also walk away from the contract and take an outside value, which is given by $U_{d}^{p}\left(A\left(Y_{t}\right)-Y_{t}\right)$. The agent's utility and continuation value are still given by (2) and (3), respectively, and his outside option is denoted as $U_{d}\left(Y_{t}\right)$. For simplicity, assume that $\rho=r$. We henceforth use $\rho$ to denote the common discount rate.

A consumption plan $C$ is sustainable if both the participation constraints for the agent (5) and the following participation constraints for the principal hold:

$$
E_{t}\left[\int_{t}^{\infty} e^{-\rho(s-t)} u^{p}\left(A\left(Y_{s}\right)-C_{s}\right) d s\right] \geq U_{d}^{p}\left(A\left(Y_{t}\right)-Y_{t}\right), \quad \forall t \geq 0 .
$$

The initial promise-keeping constraint is given by (6).

Let $\Phi(y, w)$ denote the set of all sustainable consumption plans. We can now state the primal problem as follows: 


\section{Primal problem (two-sided limited commitment):}

$$
V(y, w)=\sup _{C \in \Phi(y, w)} U^{p}(y, C)
$$

This problem may be viewed as a continuous-time version of the contracting models studied by Thomas and Worrall (1988) and Kocherlakota (1996) and discussed in Ljungqvist and Sargent (2004, Chapter 20). As in Thomas and Worrall (1988), we may interpret the principal as a risk-neutral firm and the agent as a worker. There is a competitive spot market for labor where a worker is paid $Y_{t}$ at time $t$. If the worker works for the firm, he is paid the wage $C_{t}$ at time $t$. The worker is free to walk away from the firm at any time and works in the spot market. The firm can also renege on a wage contract and buy labor at the spot market wage. Let the firm's net profit be $Y_{t}-C_{t}$ and the outside value be zero. In this case, we set $A\left(Y_{t}\right)=Y_{t}$. In Section 3.3, we will study an example of this type of models.

Following Ljungqvist and Sargent (2004, Chapter 20), we may alternatively interpret the principal and the agent as two households. Both are risk averse and the aggregate endowments $A\left(Y_{t}\right)=\bar{Y}$ are constant. In this case, the endowments of the two households are perfectly negatively correlated. The contract design problem is to find an insurance/transfer arrangement that reduces consumption risk while respecting the participation constraints for both households. In Appendix C, we will study an example of this type of models.

As in the case of one-sided limited commitment, we will solve the dual problem, and then study its relation to the primal problem.

\subsection{Duality}

As in Section 2.2, we first proceed heuristically to derive the dual problem. This heuristic derivation will not be used in our formal proofs. We write down the Lagrangian as follows,

$$
\begin{aligned}
\mathfrak{L}= & E\left[\int_{0}^{\infty} e^{-\rho t} u^{p}\left(A\left(Y_{t}\right)-C_{t}\right) d t\right]+E\left[\int_{0}^{\infty} e^{-\rho t} \lambda_{t}\left(\int_{t}^{\infty} e^{-\rho(s-t)} u\left(C_{s}\right) d s-U_{d}\left(Y_{t}\right)\right) d t\right] \\
& +E\left[\int_{0}^{\infty} e^{-\rho t} \eta_{t}\left(\int_{t}^{\infty} e^{-\rho(s-t)} u^{p}\left(A\left(Y_{s}\right)-C_{s}\right) d s-U_{d}^{p}\left(A\left(Y_{t}\right)-Y_{t}\right)\right) d t\right] \\
& +\phi\left(E\left[\int_{0}^{\infty} e^{-\rho t} u\left(C_{t}\right) d t\right]-w\right),
\end{aligned}
$$


where $e^{-\rho t} \lambda_{t}, e^{-\rho t} \eta_{t}$, and $\phi$ are the Lagrange multipliers associated with (5), (44), and (6), respectively. Integration by parts allows us to rewrite the Lagrangian as

$$
\begin{aligned}
\mathfrak{L}= & E\left[\int_{0}^{\infty} e^{-\rho t} u^{p}\left(A\left(Y_{t}\right)-C_{t}\right) d t\right]-E\left[\int_{0}^{\infty} e^{-\rho t} \lambda_{t} U_{d}\left(Y_{t}\right) d t\right] \\
& +E\left[\int_{0}^{\infty} X_{t} e^{-\rho t} u\left(C_{t}\right) d t\right]-\phi w+E\left[\int_{0}^{\infty} H_{t} e^{-\rho t} u^{p}\left(A\left(Y_{t}\right)-C_{t}\right) d t\right] \\
& -E\left[\int_{0}^{\infty} e^{-\rho t} \eta_{t} U_{d}^{p}\left(A\left(Y_{t}\right)-Y_{t}\right) d t\right],
\end{aligned}
$$

where the costate processes $\left\{X_{t}\right\}_{t \geq 0}$ and $\left\{H_{t}\right\}_{t \geq 0}$ are defined as

$$
X_{t} \equiv \int_{0}^{t} \lambda_{s} d s+\phi, \quad H_{t} \equiv \int_{0}^{t} \eta_{s} d s
$$

Define a dual function as

$$
\tilde{u}(y, x, h)=\max _{c>0}(1+h) u^{p}(A(y)-c)+x u(c) .
$$

The concavity assumption on $u$ and $u^{p}$ implies a unique solution to this problem, $c^{*}$, which satisfies the first-order condition

$$
\frac{x}{1+h}=\frac{\left(u^{p}\right)^{\prime}\left(A(y)-c^{*}\right)}{u^{\prime}\left(c^{*}\right)} .
$$

We can express $c^{*}$ as a function of $y$ and $x /(1+h)$ :

$$
c^{*}=c\left(y, \frac{x}{1+h}\right) \text {. }
$$

As in the one-sided case, we can interpret $x /(1+h)$ as the ratio of the marginal utilities of the principal and the agent (also see Ligon, Thomas, and Worrall (2002)). We can easily show that $c(y, x /(1+h))$ increases with $x /(1+h)$. It also increases with $y$ if $A$ is an increasing function of $y$. In addition, $\tilde{u}(y, x, h)$ is strictly convex in $x$ and in $h$, respectively. It is also linearly homogeneous in $x$ and $1+h$.

Optimizing with respect to $C_{t}$ in (46) yields

$$
\begin{aligned}
\mathcal{L}(X, H)= & E\left[\int_{0}^{\infty} e^{-\rho t} \tilde{u}\left(Y_{t}, X_{t}, H_{t}\right) d t\right]-E\left[\int_{0}^{\infty} e^{-\rho t} U_{d}\left(Y_{t}\right) d X_{t}\right] \\
& -E\left[\int_{0}^{\infty} e^{-\rho t} U_{d}^{p}\left(A\left(Y_{t}\right)-Y_{t}\right) d H_{t}\right]-X_{0} w .
\end{aligned}
$$


For $\mathcal{L}(X, H)$ to be finite, we impose the following integrability conditions:

$$
\begin{aligned}
E\left[\int_{0}^{\infty} e^{-\rho t}\left|U_{d}\left(Y_{t}\right)\right| d X_{t}\right] & <\infty, \\
E\left[\int_{0}^{\infty} e^{-\rho t}\left|U_{d}^{p}\left(A\left(Y_{t}\right)-Y_{t}\right)\right| d H_{t}\right] & <\infty, \\
E\left[\int_{0}^{\infty} e^{-\rho t}\left|\tilde{u}\left(X_{t}, H_{t}, Y_{t}\right)\right| d t\right] & <\infty, \\
E\left[\int_{0}^{\infty} e^{-\rho t}\left(1+H_{t}\right)\left|u^{p}\left(A\left(Y_{t}\right)\right)\right| d t\right] & <\infty .
\end{aligned}
$$

We can now formulate the dual problem by suitably choosing sets of feasible choices.

\section{Dual problem (two-sided limited commitment):}

$$
\inf _{X \in \mathcal{I}, H \in \mathcal{I}(0)} \mathcal{L}(X, H),
$$

where $\mathcal{I}(\mathcal{I}(0))$ denotes the set of all increasing processes that satisfy conditions (50)-(53), are right continuous with left limits, and start at positive values (zero).

We emphasize that in the dual problem, a feasible choice for $X$ and $H$ may not be absolutely continuous with respect to time $t$ and hence equation (47) is purely heuristic and will not be used in our formal analysis.

We solve the dual problem in two steps. First, we consider the following dual problem:

$$
\tilde{V}(y, x, h)=\inf _{X \in \mathcal{I}(x), H \in \mathcal{I}(h)} L(y, x, h, X, H),
$$

where $L(y, x, h, X, H)$ is defined as

$$
\begin{aligned}
L(y, x, h, X, H) \equiv & E\left[\int_{0}^{\infty} e^{-\rho t} \tilde{u}\left(Y_{t}, X_{t}, H_{t}\right) d t\right]-E\left[\int_{0}^{\infty} e^{-\rho t} U_{d}\left(Y_{t}\right) d X_{t}\right] \\
& -E\left[\int_{0}^{\infty} e^{-\rho t} U_{d}^{p}\left(A\left(Y_{t}\right)-Y_{t}\right) d H_{t}\right] .
\end{aligned}
$$

In this problem, we fix the initial value for the controls $X$ and $H$ at $x>0$ and $h \geq 0$, respectively. We then set $h=0$ and select the initial value $x>0$ by solving the following problem:

$$
\inf _{x>0} \tilde{V}(y, x, 0)-x w .
$$

As in the one-sided case, we can show that $\tilde{V}(y, x, h)$ is convex in $x$ by the convexity of $\tilde{u}$.

We now examine the relation between the dual and primal problems. 
Theorem 4 (weak duality) For all $C \in \Phi(y, w), X \in \mathcal{I}(x), x>0, H \in \mathcal{I}(h)$, and $h \geq 0$, the following inequality holds:

$$
U^{p}(y, C) \leq \frac{L(y, x, h, X, H)}{1+h}-\frac{x}{1+h} w
$$

Equality holds if and only if for all $t \geq 0$,

$$
\begin{aligned}
\left(1+H_{t}\right)\left(u^{p}\right)^{\prime}\left(A\left(Y_{t}\right)-C_{t}\right)-X_{t} u^{\prime}\left(C_{t}\right) & =0 \\
\int_{0}^{t} e^{-\rho s}\left(U_{s}^{a}(C)-U_{d}\left(Y_{s}\right)\right) d X_{s} & =0 \\
\int_{0}^{t} e^{-\rho s}\left(U_{s}^{p}(A(Y)-C)-U_{d}^{p}\left(A\left(Y_{s}\right)-Y_{s}\right)\right) d H_{s} & =0 .
\end{aligned}
$$

Equation (58) is the first-order condition for optimal consumption. Equations (59) and (60) are complementary slackness conditions associated with the agent's and the principal's participation constraints, respectively. From (57), we can derive that

$$
V(y, w) \leq \inf _{h>0, x>0} \frac{\tilde{V}(y, x, h)}{1+h}-\frac{x}{1+h} w .
$$

Conditions (58)-(60) are crucial to establish equality in the above equation, which is the socalled strong duality studied below.

Since $\tilde{u}(y, x, h)$ is linearly homogeneous in $(x, 1+h)$ in that $\tilde{u}(y, x, h)=(1+h) \tilde{u}\left(y, \frac{x}{1+h}, 0\right)$, so is $\tilde{V}(y, x, h)$, i.e.,

$$
\tilde{V}(y, x, h)=(1+h) \tilde{V}(y, x /(1+h), 0) .
$$

We can then define the marginal utility ratio, $X_{t} /\left(1+H_{t}\right)$, as a state variable. This property is useful to characterize the optimal contract.

The following theorem shows that the solution to the primal problem can be inferred from the solution to the dual problem.

Theorem 5 (strong duality) Let $X^{*} \in \mathcal{I}$ and $H^{*} \in \mathcal{I}(0)$ be a solution to the dual problem (54). Let $Z_{t}^{*} \equiv X_{t}^{*} /\left(1+H_{t}^{*}\right)$ and $C_{t}^{*} \equiv c\left(Y_{t}, Z_{t}^{*}\right)$. Suppose the following conditions hold: (i) $U^{p}\left(y, C^{*}\right)^{-}<\infty$. (ii) Given $H^{*}$, (52) holds for the processes $X^{\delta} \equiv X^{*}+\delta$ and $\bar{X}^{ \pm \delta} \equiv$ $X^{*}(1 \pm \delta)$ for some small $\delta>0$. (ii) Given $X^{*}$, (52) holds for the processes $H^{\delta} \equiv H^{*}+\delta$ and $\bar{H}^{ \pm \delta} \equiv H^{*}(1 \pm \delta)$ for some small $\delta>0$. Then $C^{*}$ is a solution to the primal problem (45) and

$$
V(y, w)=\inf _{z>0} \tilde{V}(y, z, 0)-z w=\inf _{x>0, h \geq 0} \frac{\tilde{V}(y, x, h)}{1+h}-\frac{x}{1+h} w .
$$


This theorem shows that, after we solve for the optimal ratio of the marginal utilities $Z_{t}^{*}$ from the dual problem, optimal consumption in the primal problem can be completely characterized by the function $c\left(Y_{t}, Z_{t}^{*}\right)$. In addition, (62) provides the link between the primal and dual value functions. In the next subsection, we use dynamic programming to characterize the dual problem and derive the solution for the marginal utility ratio.

\subsection{Dynamic Programming}

By standard theory, the variational inequality for the dual problem (55) is

$$
0=\min \left\{\tilde{V}_{x}(y, x, h)-U_{d}(y), \tilde{V}_{h}(y, x, h)-U_{d}^{p}(A(y)-y), \tilde{u}(y, x, h)+\mathcal{A} \tilde{V}(y, x, h)\right\},
$$

where

$$
\mathcal{A} \tilde{V}(y, x, h)=\tilde{V}_{y}(y, x, h) \mu(y)+\frac{1}{2} J_{y y}(y, x, h) \sigma(y)^{2}-\rho \tilde{V}(y, x, h) .
$$

Following Harrison and Taksar (1983), we construct an optimal policy as follows. There are two free boundaries satisfying, respectively,

$$
\begin{aligned}
& \tilde{V}_{x}(y, x, h)=U_{d}(y) \\
& \tilde{V}_{h}(y, x, h)=U_{d}^{p}(A(y)-y) .
\end{aligned}
$$

These two boundaries partition the state space into two types of regions. The no-jump region contains all states $(y, x, h)$ satisfying

$$
\begin{aligned}
& \tilde{u}(y, x, h)+\mathcal{A} \tilde{V}(y, x, h)=0, \\
& \tilde{V}_{x}(y, x, h)>U_{d}(y), \text { and } \tilde{V}_{h}(y, x, h)>U_{d}^{p}(A(y)-y),
\end{aligned}
$$

and the jump region contains all states $(y, x, h)$ satisfying

$$
\begin{aligned}
& \tilde{u}(y, x, h)+\mathcal{A} \tilde{V}(y, x, h)>0, \\
& \tilde{V}_{x}(y, x, h)=U_{d}(y), \text { or } \tilde{V}_{h}(y, x, h)=U_{d}^{p}(A(y)-y) .
\end{aligned}
$$

See Figure 4 in Section 3.3 for an illustration.

Because $\tilde{V}$ is linearly homogeneous in $x$ and $1+h$, we can reduce (63) to a two-dimensional problem where the state variables are $y$ and $x /(1+h)$. Since $\tilde{V}$ is convex in $x$, we can also express the two free boundaries as

$$
\frac{x}{1+h}=\varphi_{1}(y), \frac{x}{1+h}=\varphi_{2}(y),
$$

where $\varphi_{1}(y)$ and $\varphi_{2}(y)>\varphi_{1}(y)$ are determined by the value-matching conditions (64) and (65), together with super-contact conditions as illustrated in Section 3.3. 
If the initial state lies in the jump region, it jumps immediately to the no-jump region. Once the state lies in the no-jump region, the processes $X$ and $H$ are regulators such that $(Y, X, H)$ will never leave the no-jump region. The process $X$ stays constant within the no-jump region and increases if and only if $(Y, X, H)$ hits the boundary $\varphi_{1}\left(Y_{t}\right)$ at some time $t$. The process $H$ also stays constant within the no-jump region and increases if and only if $(Y, X, H)$ hits the boundary $\varphi_{2}\left(Y_{t}\right)$ at some time $t$.

Formally, we establish the following Verification Theorem analogous to Theorem 3 . The proof is also similar and hence is omitted.

Theorem 6 (verification) Suppose that there exists a twice continuously differentiable solution $\tilde{V}: \mathbb{R}_{+} \times \mathbb{R}_{++} \times \mathbb{R}_{+} \rightarrow \mathbb{R}$ to (63) such that for all $X \in \mathcal{I}(x)$ and $H \in \mathcal{I}(h), x>0$ and $h \geq 0$, (i) the process defined by

$$
\int_{0}^{t} e^{-\rho s} \tilde{V}_{y}\left(Y_{s}, X_{s}, H_{s}\right) \sigma\left(Y_{s}\right) d B_{s}, \quad t \geq 0
$$

is a martingale, and (ii) $\lim _{t \rightarrow \infty} E\left[e^{-\rho t} \tilde{V}\left(Y_{t}, X_{t}, H_{t}\right)\right]=0$. Suppose further that $X^{*} \in \mathcal{I}(x)$ and $H^{*} \in \mathcal{I}(h)$ are such that (i) for all $t>0$,

$$
0=\tilde{u}\left(Y_{t}, X_{t}^{*}, H_{t}^{*}\right)+\mathcal{A} \tilde{V}\left(Y_{t}, X_{t}^{*}, H_{t}^{*}\right)
$$

(ii) for all $t \geq 0$,

$$
\begin{aligned}
\int_{0}^{t} e^{-\rho s}\left(\tilde{V}_{x}\left(Y_{s}, X_{s}^{*}, H_{s}^{*}\right)-U_{d}\left(Y_{s}\right)\right) d X_{s}^{*} & =0 \\
\int_{0}^{t} e^{-\rho s}\left(\tilde{V}_{h}\left(Y_{s}, X_{s}^{*}, H_{s}^{*}\right)-U_{d}^{p}\left(A\left(Y_{s}\right)-Y_{s}\right)\right) d H_{s}^{*} & =0 .
\end{aligned}
$$

Then $\tilde{V}$ is the dual value function for problem (55) and $X^{*} \in \mathcal{I}(x)$ and $H^{*} \in \mathcal{I}(h)$ are the solutions to this problem. If $\tilde{V}(y, x, h)$ is strictly convex in $x$ and there exists $x^{*}>0$ such that $\tilde{V}_{x}\left(y, x^{*}, 0\right)=w$ and if the conditions in Theorem 5 are satisfied, then the primal value function is given by $V(y, w)=\tilde{V}\left(y, x^{*}, 0\right)-x^{*} w$. The optimal consumption plan and the agent's continuation value for the primal problem (7) are, respectively, given by

$$
\begin{aligned}
C_{t}^{*} & =c\left(Y_{t}, Z_{t}^{*}\right), & Z_{t}^{*} & =-V_{w}\left(Y_{t}, W_{t}^{*}\right), \\
W_{t}^{*} & =\tilde{V}_{x}\left(Y_{t}, Z_{t}^{*}, 0\right), & V\left(Y_{t}, W_{t}^{*}\right) & =\tilde{V}_{h}\left(Y_{t}, X_{t}^{*}, H_{t}^{*}\right),
\end{aligned}
$$

where $Z_{t}^{*}=X_{t}^{*} /\left(1+H_{t}^{*}\right), X_{0}^{*}=x^{*}$, and $H_{0}^{*}=0$.

Equation (69) shows that optimal consumption can be characterized by two state variables, the income level $Y_{t}$ and the marginal utility ratio $Z_{t}^{*}=X_{t}^{*} /\left(1+H_{t}^{*}\right)$. Applying the envelope 
condition to (62), we deduce that the marginal utility ratio $Z_{t}^{*}$ is equal to the negative slope of the Pareto frontier, $-V_{w}\left(Y_{t}, W_{t}^{*}\right)$. Applying the first-order condition to (62), the agent's continuation value can be characterized by the partial derivative of the dual value function. This result is similar to that in the case of one-sided limited commitment.

Differentiating (61) with respect to $h$ yields:

$$
\tilde{V}_{h}(y, x, h)=\tilde{V}(y, x /(1+h), 0)-\tilde{V}_{x}(y, x /(1+h), 0) \frac{x}{1+h} .
$$

Thus, we can also describe the principal's value using the partial derivative of the dual value function:

$$
\tilde{V}_{h}\left(Y_{t}, X_{t}^{*}, H_{t}^{*}\right)=\tilde{V}\left(Y_{t}, Z_{t}^{*}, 0\right)-\tilde{V}_{x}\left(Y_{t}, Z_{t}^{*}, 0\right) Z_{t}^{*}=\tilde{V}\left(Y_{t}, Z_{t}^{*}, 0\right)-W_{t}^{*} Z_{t}^{*}=V\left(Y_{t}, W_{t}^{*}\right)
$$

Similar to Theorem 3 in the one-sided limited commitment case, condition (67) indicates that the process $X^{*}$ increases if and only if $\tilde{V}_{x}\left(Y_{t}, X_{t}^{*}, H_{t}^{*}\right)=U_{d}\left(Y_{t}\right)=W_{t}^{*}$. In addition, condition (68) indicates that $H_{t}^{*}$ increases if and only if $\tilde{V}_{h}\left(Y_{t}, X_{t}^{*}, H_{t}^{*}\right)=U_{d}^{p}\left(A\left(Y_{t}\right)-Y_{t}\right)=V\left(Y_{t}, W_{t}^{*}\right)$. These two conditions are the complementary slackness conditions associated with the agent's and the principal's participation constraints. We can equivalently express the solution $X^{*}$ and $H^{*}$ as

$$
\begin{aligned}
& X_{t}^{*}=\max \left\{x^{*}, \sup _{s \in[0, t]} \varphi_{1}\left(Y_{s}\right)\left(1+H_{s}^{*}\right)\right\}, \\
& H_{t}^{*}=\max \left\{0, \sup _{s \in[0, t]} \frac{X_{s}^{*}}{\varphi_{2}\left(Y_{s}\right)}-1\right\} .
\end{aligned}
$$

Since $\tilde{V}_{x}\left(y, \varphi_{1}(y), 0\right)=U_{d}(y)$ on the lower boundary, it follows from $w \geq U_{d}\left(Y_{0}\right)$ that

$$
\tilde{V}_{x}\left(Y_{0}, X_{0}^{*}, 0\right)=w \geq \tilde{V}_{x}\left(Y_{0}, \varphi_{1}\left(Y_{0}\right), 0\right) .
$$

If $\tilde{V}(y, x, h)$ is convex in $x$, then $X_{0}^{*} \geq \varphi_{1}\left(Y_{0}\right)$. If $w$ is sufficiently large, then $X_{0}^{*}$ is also sufficiently large by the convexity of $\tilde{V}(\cdot, x, \cdot)$ in $x$. Since we can show that $\tilde{V}_{h}\left(Y_{0}, X_{0}, 0\right)$ decreases in $X_{0}{ }^{19} \tilde{V}_{h}\left(Y_{0}, X_{0}, 0\right)$ will fall below $U_{d}^{p}\left(A\left(Y_{0}\right)-Y_{0}\right)$ when $X_{0}$ is sufficiently large. In this case, the principal's participation constraint is violated. Thus, there must be an upper

${ }^{19}$ Differentiating $(61)$ with respect to $h$ yields:

$$
\tilde{V}_{h}(y, x, h)=\tilde{V}(y, x /(1+h), 0)-\tilde{V}_{x}(y, x /(1+h), 0) \frac{x}{1+h} .
$$

Differentiating with respect to $x$ yields:

$$
\tilde{V}_{h x}(y, x, h)=\tilde{V}_{x}(y, x /(1+h), 0) \frac{1}{1+h}-\tilde{V}_{x x} \frac{x}{(1+h)^{2}}-\tilde{V}_{x}(y, x /(1+h), 0) \frac{1}{1+h}=-V_{x x} \frac{x}{(1+h)^{2}}<0 .
$$


bound on $w$. The upper bound is given by $\bar{w}=\tilde{V}_{x}\left(Y_{0}, \varphi_{2}\left(Y_{0}\right), 0\right)$, because if $w$ exceeds $\bar{w}$, then $X_{0}>\varphi_{2}\left(Y_{0}\right)$ and $\tilde{V}_{h}\left(Y_{0}, X_{0}, 0\right)<U_{d}^{p}\left(A\left(Y_{0}\right)-Y_{0}\right)$. Consequently, we must have $w \in$ $\left[U_{d}\left(Y_{0}\right), \bar{w}\right]$. In this case, the state vector $\left(Y_{t}, X_{t}^{*}, H_{t}^{*}\right)$ always lies in the no-jump region and both $X^{*}$ and $H^{*}$ are continuous processes.

\subsection{Example II}

In this section, we introduce limited commitment from the principal's side in Example I of Section 2.5. Suppose the principal's outside option is autarky as well. The agent has income $Y_{t}$ given by (33) and the principal does not have any income. Thus, the risk-neutral principal's autarky value is zero and the agent's autarky value is given by (34). Recall that the agent's utility is given by $u(c)=c^{\alpha} / \alpha, 0 \neq \alpha<1$, and we still maintain the same assumptions on parameter values as in Section 2.5. Note that our solution for the policy functions in the dual problem when $\alpha=0$ applies to the case of logarithmic utility $u(c)=\ln c^{20}$

We can compute the dual function as

$$
\tilde{u}(y, x, h)=\max _{c>0}(1+h)(y-c)+x u(c)=(1+h) y+\frac{1-\alpha}{\alpha} x^{\frac{1}{1-\alpha}}(1+h)^{\frac{-\alpha}{1-\alpha}},
$$

and the optimal consumption rule as

$$
c^{*}=\left(\frac{x}{1+h}\right)^{\frac{1}{1-\alpha}}
$$

\subsubsection{Solution}

Hinted by the solution in Section 2.5, we conjecture that the no-jump region is given by

$$
\left\{(y, x, h) \in \mathbb{R}_{++} \times \mathbb{R}_{++} \times \mathbb{R}_{+}: \frac{x}{1+h} \in\left[b_{1} y^{1-\alpha}, b_{2} y^{1-\alpha}\right]\right\},
$$

where $0<b_{1}<b_{2}$ are to be determined. We can verify that the dual value function with the following form satisfies (66) in the no-jump region,

$$
\begin{aligned}
\tilde{V}(y, x, h)= & \frac{1-\alpha}{\alpha \rho}(1+h)\left(\frac{x}{1+h}\right)^{\frac{1}{1-\alpha}}+\frac{(1+h) y}{\rho-\mu} \\
& +A_{1}(1-\alpha)(1+h)\left(\frac{x}{1+h}\right)^{\frac{1-\beta_{1}}{1-\alpha}} y^{\beta_{1}} \\
& +A_{2}(1-\alpha)(1+h)\left(\frac{x}{1+h}\right)^{\frac{1-\beta_{2}}{1-\alpha}} y^{\beta_{2}}
\end{aligned}
$$

\footnotetext{
${ }^{20}$ For log utility, the dual value function is given by

$$
\tilde{V}(y, x, h)=\frac{1}{\rho}\left[x \ln \left(\frac{x}{1+h}\right)-x\right]+\frac{(1+h) y}{\rho-\mu}+A_{1} x^{1-\beta_{1}}(1+h)^{\beta_{1}} y^{\beta_{1}}+A_{2}(1+h)^{\beta_{2}} x^{1-\beta_{2}} y^{\beta_{2}},
$$
}

where $A_{1}$ and $A_{2}$ are constants determined by the boundary conditions. It is not the limit when $\alpha \rightarrow 0$. 
where $\beta_{1}$ and $\beta_{2}>\beta_{1}$ are the two roots of equation (38) for $r=\rho$ and $A_{1}$ and $A_{2}$ are constants to be determined. We can also verify that the dual value function in the jump region takes the following form: for $x /(1+h)>b_{2} y^{1-\alpha}$,

$$
\tilde{V}(y, x, h)=\tilde{V}\left(y, x, \frac{x}{b_{2} y^{1-\alpha}}-1\right),
$$

and for $x /(1+h)<b_{1} y^{1-\alpha}$,

$$
\tilde{V}(y, x, h)=\tilde{V}\left(y, b_{1} y^{1-\alpha}(1+h), h\right)+\left(x-b_{1} y^{1-\alpha}(1+h)\right) U_{d}(y)
$$

We use the following four value-matching and super-contact conditions to determine the four constants $A_{1}, A_{2}, b_{1}$, and $b_{2}$ :

$$
\begin{array}{rlrl}
\lim _{\frac{x}{1+h} \downarrow b_{1} y^{1-\alpha}} \tilde{V}_{x}(y, x, h) & =U_{d}(y), & \lim _{\frac{x}{1+h} \uparrow b_{2} y^{1-\alpha}} \tilde{V}_{h}(y, x, h)=0, \\
\lim _{\frac{x}{1+h} \downarrow b_{1} y^{1-\alpha}} \tilde{V}_{x x}(y, x, h) & =0, & \frac{x}{1+h} \uparrow b_{2} y^{1-\alpha} & \lim _{h h}(y, x, h)=0 .
\end{array}
$$

We can simplify the above four equations to two equations for $b_{1}$ and $b_{2}$.

Proposition 2 Suppose that $\alpha<1, \sigma>0, \rho>\mu+\sigma^{2} / 2$, and $\rho>\alpha \mu+\alpha(\alpha-1) \sigma^{2} / 2$. Then there are two solutions for $b_{1}$ and $b_{2}$. One solution is such that $0<b_{1}<1<b_{2}<$ $\left(\frac{\rho}{\rho-\mu} \frac{1-\beta_{1}}{-\beta_{1}}\right)^{1-\alpha}$. The other solution is degenerate $\left(b_{1}=b_{2}=1\right)$.

We rule out the degenerate solution since there are no two increasing processes $X^{*}$ and $H^{*}$ satisfying $X^{*}=\left(1+H^{*}\right) Y^{1-\alpha}$ for a geometric Brownian motion $Y$.

\subsubsection{Numerical Illustrations}

Figure 4 plots the state space. It shows that the two free boundaries $x /(1+h)=b_{1} y^{1-\alpha}$ and $x /(1+h)=b_{2} y^{1-\alpha}$ partition the state space into three areas. The area inside the two boundaries is the no-jump region and the other two areas are the jump region. The initial state $\left(Y_{0}, X_{0}^{*}\right)$ is inside the no-jump region. Consumption is constant in the interior of the no-jump region. Whenever $Y_{t}$ increases to the lower boundary, $X_{t}^{*}$ and $C_{t}^{*}$ rise, but $H_{t}^{*}$ does not change. Whenever $Y_{t}$ decreases to the upper boundary, $H_{t}^{*}$ rises and $C_{t}^{*}$ falls, but $X_{t}^{*}$ does not change.

Figure 5 plots the dual value function $\tilde{V}(y, x, 0)$ and the primal value function $V(y, w)$ for three values $y \in\{0.9,1,1.1\}$ in the no-jump region. This figure shows that $\tilde{V}(y, x, 0)$ is strictly convex in $x$ and $V(y, w)$ is strictly concave and decreasing in $w$. Note that the domains for both functions change with $y$. In particular, the domain of $V(y, w)$ for $w$ increases with $y$ because a larger promised value is needed to induce the agent's participation when his income is larger. 


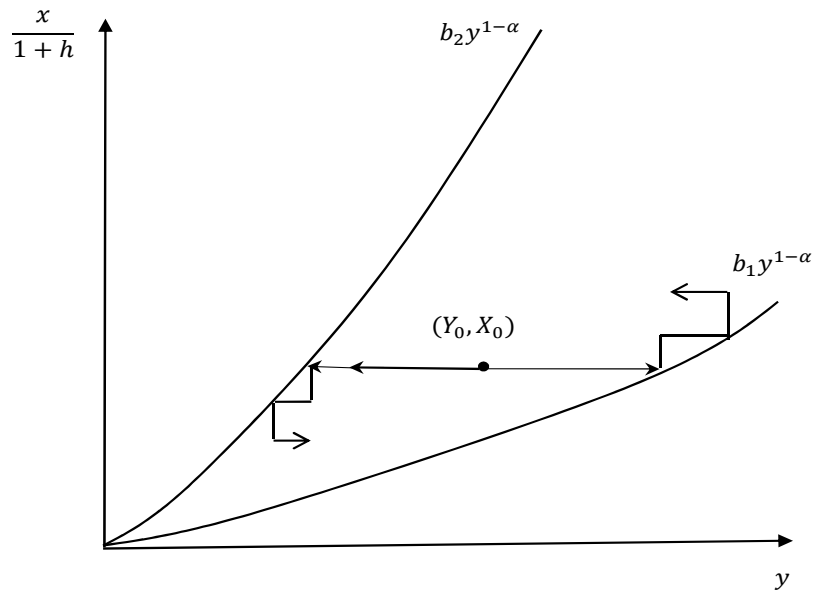

Figure 4: The state space for Example II. The two curves $x /(1+h)=b_{1} y^{1-\alpha}$ and $x /(1+h)=$ $b_{2} y^{1-\alpha}$ partition the state space into three areas. The middle area is the no-jump region and the other two areas are the jump region.
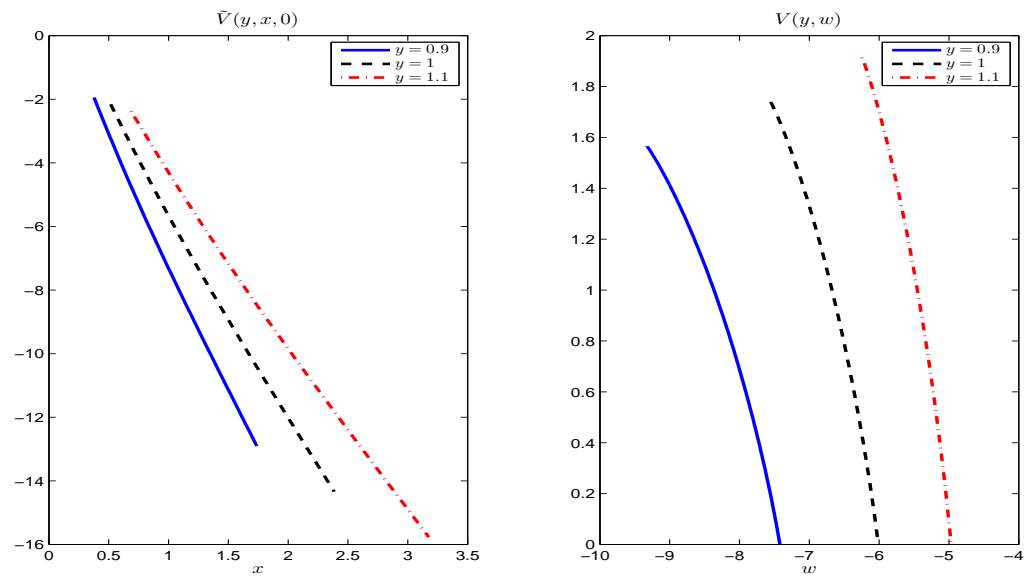

Figure 5: The dual and primal value functions in the no-jump region for Example II. Parameter values are given by $\mu=0.02, \sigma=0.1, \rho=0.04$, and $\alpha=-2$. 

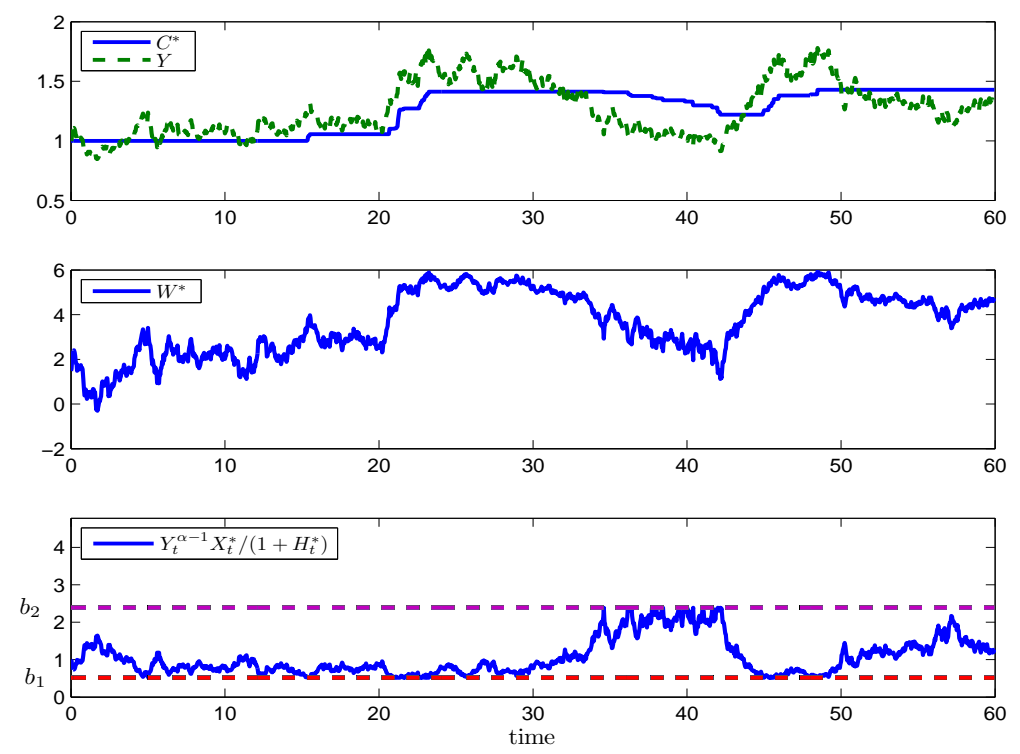

Figure 6: Simulated paths of the agent's optimal consumption $C_{t}^{*}$, incomes $Y_{t}$, continuation values $W_{t}^{*}$, and the process $Y_{t}^{\alpha-1} X_{t}^{*} /\left(1+H_{t}^{*}\right), t \geq 0$, for Example II. Parameter values are given by $\mu=0.02, \sigma=0.1, \rho=0.04$, and $\alpha=-2$.

By Harrison and Taksar (1983), Harrison (1985), or Stokey (2008), we deduce that $\ln X^{*}$ and $\ln H^{*}$ regulate the reflected diffusion process,

$$
(\alpha-1) \ln Y_{t}+\ln X_{t}^{*}-\ln \left(1+H_{t}^{*}\right), \quad t \geq 0,
$$

within the band $\left[\ln b_{1}, \ln b_{2}\right]$. We can then express the solutions for $X^{*}$ and $H^{*}$ as

$$
\begin{aligned}
& X_{t}^{*}=\max \left\{x^{*}, \sup _{s \in[0, t]} b_{1} Y_{s}^{1-\alpha}\left(1+H_{s}^{*}\right)\right\}, \\
& H_{t}^{*}=\max \left\{0, \sup _{s \in[0, t]} \frac{X_{s}^{*}}{b_{2} Y_{s}^{1-\alpha}}-1\right\},
\end{aligned}
$$

where $X_{0}^{*}=x^{*}$ and $H_{0}^{*}=0$. Figure 6 plots the simulated paths of incomes $Y_{t}$, consumption $C_{t}^{*}=\left(X_{t}^{*} /\left(1+H_{t}^{*}\right)\right)^{\frac{1}{1-\alpha}}$, the continuation value $W_{t}^{*}=\tilde{V}_{x}\left(Y_{t}, X_{t}^{*} /\left(1+H_{t}^{*}\right), 0\right)$, and $X_{t}^{*} /\left(1+H_{t}^{*}\right) Y_{t}^{\alpha-1}$. This figure shows intuitively how $C_{t}^{*}$ and $W_{t}^{*}$ move with incomes $Y_{t}$. Using (70), we can show that $W_{t}^{*}$ normalized by the autarky value $\kappa Y_{t}^{\alpha}$ is an invertible function of $C_{t}^{*} / Y_{t}$. Thus, we can also write $C_{t}^{*} / Y_{t}$ as a function of $W_{t}^{*} /\left(\kappa Y_{t}^{\alpha}\right)$, which may be derived as in the standard approach using the continuation value as a state variable.

Unlike the solution with one-sided limited commitment, the optimal consumption allocation with two-sided limited commitment can either increase or decrease depending on income realizations. This is more consistent with the empirical evidence on consumption reported in Ligon, 
Thomas, and Worrall (2002). When our model is interpreted as a model of wage contracts as in Thomas and Worrall (1988), our result implies that contract wages are procyclical and sticky in response to spot market wages.

\subsubsection{Stationary Distribution}

Since $C_{t}^{*}=\left(X_{t}^{*} /\left(1+H_{t}^{*}\right)\right)^{\frac{1}{1-\alpha}}$, it follows that $\ln \left(C_{t}^{*} / Y_{t}\right)$ is a regulated Brownian motion with drift $\sigma^{2} / 2-\mu$ and volatility $-\sigma$ on $\left[(1-\alpha)^{-1} \ln b_{1},(1-\alpha)^{-1} \ln b_{2}\right]$. By Proposition 5.5 in Harrison (1985) or Proposition 10.8 in Stokey (2008), $\ln \left(C_{t}^{*} / Y_{t}\right)$ has a stationary distribution with the density function

$$
p(x)=\frac{\delta e^{\delta x}}{b_{2}^{\frac{\delta}{1-\alpha}}-b_{1}^{\frac{\delta}{1-\alpha}}}, \quad x \in\left[(1-\alpha)^{-1} \ln b_{1},(1-\alpha)^{-1} \ln b_{2}\right],
$$

where $\delta \equiv 1-2 \mu / \sigma^{2}$.

In a discrete-time setup with a symmetric IID endowment process, Kocherlakota (1996) shows that there is a unique long-run stationary distribution for the agent's continuation values. In our continuous-time model, the agent's endowment is a geometric Brownian motion process. We can show that the agent's continuation value $W_{t}^{*}$ normalized by his autarky value $\kappa Y_{t}^{\alpha}$ has a unique long-run stationary distribution because we have shown before that $W_{t}^{*} /\left(\kappa Y_{t}^{\alpha}\right)$ can be written as a function of $C_{t}^{*} / Y_{t}$.

By Proposition 13 in Chapter 5 of Harrison (1985) or Stokey (2008), the average increase and the average decrease in $\ln \left(C_{t}^{*} / Y_{t}\right)$ per unit of time are, respectively, given by

$$
\frac{\left(\sigma^{2} / 2-\mu\right) b_{1}^{\frac{\delta}{1-\alpha}}}{b_{2}^{\frac{\delta}{1-\alpha}}-b_{1}^{\frac{\delta}{1-\alpha}}} \text { and } \frac{\left(\sigma^{2} / 2-\mu\right) b_{2}^{\frac{\delta}{1-\alpha}}}{b_{2}^{\frac{\delta}{1-\alpha}}-b_{1}^{\frac{\delta}{1-\alpha}}}
$$

It follows that the average increase per unit of time is higher than the average decrease per unit of time if and only if $\sigma^{2} / 2<\mu$.

The above analytical results can be tested if one has good individual data. When our model is interpreted as a model of wage contracts, if we have data on individual productivity, we can use (74) to calculate the frequency of wage changes in the model and compare it with the wage rigidity observed in the data. When our model is interpreted as a consumption insurance model, if we have data on individual income and consumption, we can compare the empirical distribution of consumption-income ratio with that derived in (73). Finally, if we have long times-series data on income and consumption, we may test whether $X_{t}^{*}$ and $H_{t}^{*}$ (defined in (71) and $(72))$ are sufficient statistics for $C_{t}$. In particular, we can regress $C_{t}$ on both $\left(X_{t}^{*}, H_{t}^{*}\right)$ and other lagged variables in the income process, and test whether the coefficient on other variables are zero or not. 

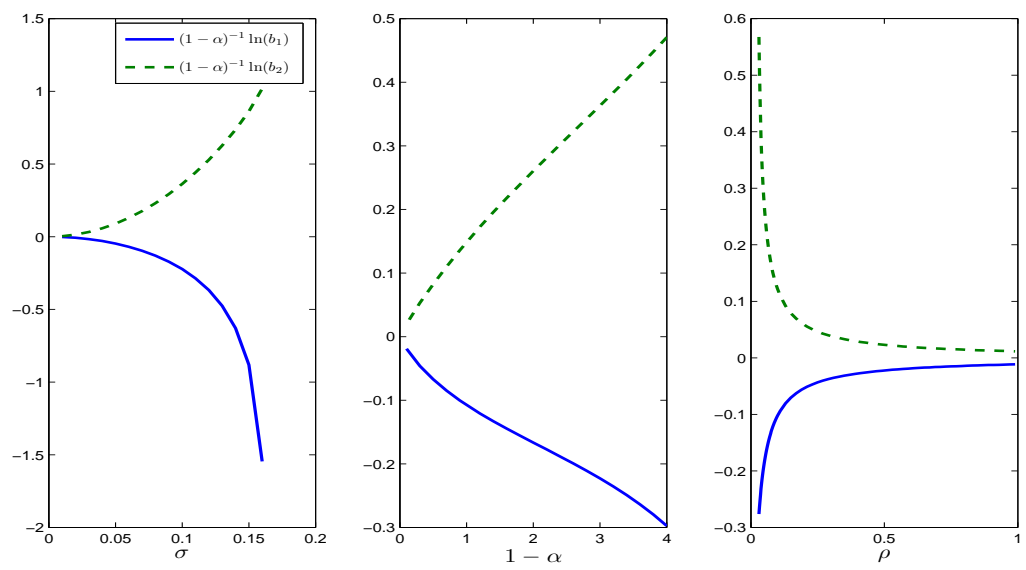

Figure 7: Comparative statics for Example II. Parameter values are given by $\mu=0.02, \sigma=0.1$, $\rho=0.04$, and $\alpha=-2$, unless one of them is changed in the comparative statics.

\subsubsection{Comparative Statics}

First, we consider the effect of the volatility of the agent's income on risk sharing. Because the agent is risk averse, a larger volatility reduces his autarky value. A lower autarky value reduces the agent's incentive to default, and hence makes it easier to enforce risk-sharing contracts. In line with this intuition, the left panel of Figure 7 shows that the risk-sharing band expands with $\sigma$.

Next we study how the agent's risk aversion affects risk sharing. When the agent is more risk averse (i.e., when $1-\alpha$ is higher), then he is less willing to go to autarky because in autarky he must face full income uncertainty. Similar to the above, risk sharing becomes easier when the agent's incentive to default is reduced. Consistent with this intuition, the middle panel of Figure 7 shows that the risk-sharing band expands as $1-\alpha$ increases. On the other hand, the band shrinks to a singleton (i.e., autarky) as $\alpha \rightarrow 1$. This is because the agent is risk-neutral if $\alpha=1$, in which case autarky is the only enforceable allocation. When both the principal and the agent are risk neutral, any contract is a zero-sum game. That is, any contract that gives one party a positive net gain (over autarky) must impose a loss upon the other party. The latter would default.

Finally, we discuss the effect of the subjective discount rate $\rho$. Consider the party who is making a transfer to his partner. To satisfy his participation constraint, the benefit of future insurance must exceed the current loss. Hence, higher patience (i.e., lower $\rho$ ) increases the weight on future benefit and makes it easier to satisfy the participation constraint. Consistent 
with this intuition, the right panel of Figure 7 shows that the risk-sharing band expands as $\rho$ decreases. The result that patience enhances cooperation is well known in the literature on models with limited commitment, as well as in game theory.

\subsubsection{Why Are Continuous-Time Models Different?}

Our comparative statics results are generally consistent with those in discrete-time models. However, there is an important difference. In discrete-time models, Kocherlakota (1996), Alvarez and Jermann (2000, 2001), and Ligon, Thomas, and Worrall (2002) show that there may be three regimes for efficient allocation depending on parameter values: (i) full risk sharing forever is possible; (ii) only limited risk sharing is possible; or (iii) only autarky is possible. In particular, for high enough values of the discount factor, sufficient endowment risk, or enough degree of risk aversion, the first-best allocation is an optimal contract. In the opposite extreme, the autarky allocation is optimal. By contrast, Proposition 2 shows that the risk-sharing band is finite for any admissible parameter values satisfying the assumptions in this proposition. Thus, neither the first-best allocation nor autarky can be an optimal contract. This difference is due to our continuous-time Brownian motion environment. In the existing literature on discretetime models, income processes are often assumed to be either IID or stationary Markovian with a bounded support. In our model, the income process is a nonstationary geometric Brownian motion.

To better understand the difference, we discretize Example II for the special case with $u(c)=\ln c$ and $\mu=0$. A time interval is denoted by $d t$ and the income process is approximated by a binomial process,

$$
\frac{y_{t+d t}-y_{t}}{y_{t}}= \begin{cases}\sigma \sqrt{d t} & \text { with probability } 0.5 \\ -\sigma \sqrt{d t} & \text { with probability } 0.5\end{cases}
$$

An important feature of this shock is that its standard deviation is $\sigma \sqrt{d t}$. The autarky value $U_{d}(y)$ satisfies the recursion:

$$
U_{d}(y)=\ln (y) d t+\frac{1}{1+\rho d t}\left[0.5 U_{d}((1+\sigma \sqrt{d t}) y)+0.5 U_{d}((1-\sigma \sqrt{d t}) y)\right] .
$$

Following Ljungqvist and Sargent (2004), we write down the discrete-time Bellman equation as

$$
\begin{aligned}
U^{p}(y, v)=\max _{c, v_{b}, v_{g}} & (y-c) d t+\frac{1}{1+\rho d t}\left[0.5 U^{p}\left((1-\sigma \sqrt{d t}) y, v_{b}\right)+0.5 U^{p}\left((1+\sigma \sqrt{d t}) y, v_{g}\right)\right] \\
\text { subject to } & v=(\ln (c)-\ln (y)) d t+\frac{1}{1+\rho d t}\left(0.5 v_{b}+0.5 v_{g}\right), \\
& v_{b} \in\left[0, \bar{U}^{a}((1-\sigma \sqrt{d t}) y)\right], \\
& v_{g} \in\left[0, \bar{U}^{a}((1+\sigma \sqrt{d t}) y)\right],
\end{aligned}
$$


where $U^{p}(y, v)$ is the principal's value function when the income is $y$ and the surplus of the agent's continuation value over his autarky value is $v$. Here, $v_{b}$ and $v_{g}$ denote the agent's surplus in the continuation contract after a bad and a good shock, respectively, and $\bar{U}^{a}(y)$ denotes the agent's highest surplus among all sustainable contracts when his income is $y$. Since $U^{p}(y, v)$ is decreasing in $v, U^{p}\left(y, \bar{U}^{a}(y)\right)=0$ by the principal's participation constraint. When $d t=1$, we obtain a discrete-time model.

Any full risk sharing allocation gives a constant continuation value to the agent. When $y$ is sufficiently large, the autarky value $U_{d}(y)$ may exceed this value, violating the agent's participation constraint. Thus, full risk sharing is not sustainable even in discrete time. This result is generally true when the income process is unbounded. However, it is more subtle to understand why autarky is never optimal in our continuous-time model but it may be optimal in the discrete-time model depending on parameter values. In Appendix A, we prove the following result.

Proposition 3 In the discretized model of example II, a non-autarkic sustainable risk sharing contract exists if and only if $\sigma \sqrt{d t}>2 \rho d t$.

This proposition states that in the discretized model, a non-autarkic risk sharing contract exists if and only if the income volatility, $\sigma \sqrt{d t}$, is sufficiently large or the agent is sufficiently patient (i.e., $\rho d t$ is so small that the discount factor $1 /(1+\rho d t)$ is sufficiently close to 1$)$. This means that autarky is the only optimal contract if and only if the income process is not too volatile or the agent is sufficiently impatient. This result is consistent with the finding in the literature of discrete-time models and does not depend on whether the income process is stationary or not. By contrast, when the time interval is sufficiently small, the condition $\sigma \sqrt{d t}>2 \rho d t$ is always satisfied, and hence autarky cannot be an optimal contract. ${ }^{21}$ This implies that the discreteness of the time grid is responsible for the key differences between our continuous time model and discrete time models.

The idea of the proof is as follows. We construct a particular risk sharing contract in which the principal receives a small amount of income from the agent if a good shock realizes, but transfers a small amount of income to the agent, otherwise. This contract is such that it always satisfies the agent's participation constraints, but whether it satisfies the principal's participation constraints depends on the time interval $d t$. Specifically, we show in Appendix A that the net benefit over autarky to the principal from the constructed contract is positively related to $\sigma \sqrt{d t}-2 \rho d t$. When $d t$ is sufficiently small, the net benefit is always positive.

\footnotetext{
${ }^{21}$ Note that we do not take the continuous time limit as $d t \rightarrow 0$. It is technically involved to prove the convergence from the discretized model to the continuous-time model. Such an analysis is beyond the scope of this paper.
} 


\section{Conclusion}

In this paper, we have proposed a duality approach to solving continuous-time contracting problems with either one-sided or two-sided limited commitment. We have established the weak and strong duality theorems and provided a dynamic programming characterization of the dual problem. We have also provided explicit solutions for two examples of a consumption insurance problem. We have demonstrated how our approach is analytically convenient and how the optimal contracts in continuous time are different from those in discrete time. In particular, we have shown that neither autarky nor full risk sharing can be an optimal contract with two-sided limited commitment, unlike in discrete-time models. An important advantage of our approach over the standard approach using the continuation value as a state variable is that our state space is the positive orthant, but the state space for the continuation value is endogenous under two-sided limited commitment. The other advantage is that the HJB equation in the dual problem is a linear PDE, while that in the primal problem is a nonlinear PDE with state constraints. An interesting direction of future research is to apply the duality approach to other contracting environments, such as those with moral hazard or adverse selection. For instance, Pavan, Segal, and Toikka (2014) and Bergemann and Strack (2014) use the first-order approach to study dynamic mechanism design problems in which incentive constraints are replaced by first-order conditions. Their relaxed problem may be solved using the duality approach. 


\section{Appendices}

\section{A Proofs}

The following lemma will be repeatedly used in later proofs.

Lemma 1 Define a process $M$ by

$$
M_{t}=E_{t}\left[\int_{t}^{\infty} e^{-\rho(s-t)} N_{s} d s\right] .
$$

If one of the two conditions is satisfied for $X \in \mathcal{I}$,

1. $E\left[\int_{0}^{\infty} e^{-\rho t} X_{t}\left|N_{t}\right| d t\right]<\infty$,

2. $\left\{N_{t}\right\}_{t \geq 0}$ is nonnegative and $E\left[\int_{0}^{\infty} e^{-\rho t} M_{t} d X_{t}\right]<\infty$,

then

$$
E\left[\int_{0}^{\infty} e^{-\rho t} X_{t} N_{t} d t\right]=X_{0} M_{0}+E\left[\int_{0}^{\infty} e^{-\rho t} M_{t} d X_{t}\right]
$$

Proof: For any finite $T>0$, integration by parts yields:

$$
\begin{aligned}
& E\left[\int_{0}^{T} e^{-\rho t} X_{t} N_{t} d t\right] \\
= & -E\left[\int_{0}^{T} X_{t} d\left(\int_{t}^{T} e^{-\rho s} N_{s} d s\right)\right] \\
= & -E\left[\left.\left(X_{t} \int_{t}^{T} e^{-\rho s} N_{s} d s\right)\right|_{0} ^{T}-\int_{0}^{T}\left(\int_{t}^{T} e^{-\rho s} N_{s} d s\right) d X_{t}\right] \\
= & X_{0} E\left[\int_{0}^{T} e^{-\rho s} N_{s} d s\right]+E\left[\int_{0}^{T} e^{-\rho t} E_{t}\left[\int_{t}^{T} e^{-\rho(s-t)} N_{s} d s\right] d X_{t}\right],
\end{aligned}
$$

where the last equality follows from the Law of Iterative Expectations. If $\left\{N_{t}\right\}_{t \geq 0}$ is nonnegative and $E\left[\int_{0}^{\infty} e^{-\rho t} M_{t} d X_{t}\right]<\infty$, then the Monotone Convergence Theorem implies that

$$
\begin{aligned}
E\left[\int_{0}^{\infty} e^{-\rho t} X_{t} N_{t} d t\right] & =\lim _{T \rightarrow \infty} E\left[\int_{0}^{T} e^{-\rho t} X_{t} N_{t} d t\right] \\
& =X_{0} E\left[\int_{0}^{\infty} e^{-\rho s} N_{s} d s\right]+\lim _{T \rightarrow \infty} E\left[\int_{0}^{T} e^{-\rho t} E_{t}\left[\int_{t}^{T} e^{-\rho(s-t)} N_{s} d s\right] d X_{t}\right] \\
& =X_{0} E\left[\int_{0}^{\infty} e^{-\rho s} N_{s} d s\right]+E\left[\int_{0}^{\infty} e^{-\rho t} E_{t}\left[\int_{t}^{\infty} e^{-\rho(s-t)} N_{s} d s\right] d X_{t}\right] .
\end{aligned}
$$

If $E\left[\int_{0}^{\infty} e^{-\rho t} X_{t}\left|N_{t}\right| d t\right]<\infty$, then the same conclusion follows from the Dominated Convergence Theorem. Q.E.D. 
Proof of Proposition 1: Let $\theta \in(0,1)$. For any $x_{1}, x_{2}>0$ and any two processes $X_{1} \in$ $\mathcal{I}\left(x_{1}\right)$ and $X_{2} \in \mathcal{I}\left(x_{2}\right)$, define $x^{\theta} \equiv \theta x_{1}+(1-\theta) x_{2}$ and define the process $X^{\theta}$ as $X_{t}^{\theta} \equiv$ $\theta X_{1 t}+(1-\theta) X_{2 t}$. By the definition of $\tilde{u}$ and the convexity of $\tilde{u}$,

$$
X_{t}^{\theta} e^{-(\rho-r) t} u\left(C_{t}\right)-C_{t} \leq \tilde{u}\left(e^{-(\rho-r) t} X_{t}^{\theta}\right) \leq \theta \tilde{u}\left(e^{-(\rho-r) t} X_{1 t}\right)+(1-\theta) \tilde{u}\left(e^{-(\rho-r) t} X_{2 t}\right)
$$

for any $C \in \Gamma(y, w)$. From the proof of Theorem 1 below, we have

$$
E\left[\int_{0}^{\infty} e^{-\rho t} X_{1 t}\left|u\left(C_{t}\right)\right| d t\right]<\infty \quad \text { and } \quad E\left[\int_{0}^{\infty} e^{-\rho t} X_{2 t}\left|u\left(C_{t}\right)\right| d t\right]<\infty .
$$

In addition, it follows from (16) that

$$
E\left[\int e^{-r t}\left|\tilde{u}\left(e^{-(\rho-r) t} X_{1 t}\right)\right| d t\right]<\infty \quad \text { and } \quad E\left[\int e^{-r t}\left|\tilde{u}\left(e^{-(\rho-r) t} X_{2 t}\right)\right| d t\right]<\infty .
$$

Thus, $X^{\theta}$ satisfies the integrability condition (16). It is also trivial to verify that $X^{\theta}$ satisfies the integrability condition (15). Consequently, $X^{\theta} \in \mathcal{I}\left(x^{\theta}\right)$.

It follows from the convexity of $\tilde{u}$ that

$$
L\left(y, x^{\theta}, X^{\theta}\right) \leq \theta L\left(y, x_{1}, X_{1}\right)+(1-\theta) L\left(y, x_{2}, X_{2}\right)
$$

Since $X^{\theta} \in \mathcal{I}\left(x^{\theta}\right)$,

$$
\tilde{V}\left(y, x^{\theta}\right) \leq L\left(y, x^{\theta}, X^{\theta}\right) \leq \theta L\left(y, x_{1}, X_{1}\right)+(1-\theta) L\left(y, x_{2}, X_{2}\right) .
$$

Taking infimum yields

$$
\begin{aligned}
\tilde{V}\left(y, x^{\theta}\right) & \leq \theta \inf _{X_{1} \in \mathcal{I}\left(x_{1}\right)} L\left(y, x_{1}, X_{1}\right)+(1-\theta) \inf _{X_{2} \in \mathcal{I}\left(x_{2}\right)} L\left(y, x_{2}, X_{2}\right) \\
& =\theta \tilde{V}\left(y, x_{1}\right)+(1-\theta) \tilde{V}\left(y, x_{2}\right)
\end{aligned}
$$

as desired. Q.E.D.

Proof of Theorem 1: By the definition of $\tilde{u}$ in (12),

$$
X_{t} e^{-(\rho-r) t} u\left(C_{t}\right)-C_{t} \leq \tilde{u}\left(e^{-(\rho-r) t} X_{t}\right),
$$

for any $C \in \Gamma(y, w)$. By (A.1), (1), and (16), we deduce that $E\left[\int_{0}^{\infty} e^{-\rho t} X_{t}\left(u\left(C_{t}\right)\right)^{+} d t\right]<\infty$, where we use $u^{+}$and $u^{-}$to denote the positive and negative parts, respectively, of any $u \in \mathbb{R}$. By Lemma 1,

$$
E\left[\int_{0}^{\infty}\left(\int_{t}^{\infty} e^{-\rho s}\left(u\left(C_{s}\right)\right)^{+} d s\right) d X_{t}\right]<\infty
$$


It follows from $U_{t}^{a}(C) \geq U_{d}\left(Y_{t}\right)$ and $E\left[\int_{0}^{\infty} e^{-\rho t}\left|U_{d}\left(Y_{t}\right)\right| d X_{t}\right]<\infty$ that

$$
\begin{aligned}
& E\left[\int_{0}^{\infty}\left(\int_{t}^{\infty} e^{-\rho s}\left(u\left(C_{s}\right)\right)^{-} d s\right) d X_{t}\right] \\
\leq & E\left[\int_{0}^{\infty}\left(\int_{t}^{\infty} e^{-\rho s}\left(u\left(C_{s}\right)\right)^{+} d s\right) d X_{t}\right]-E\left[\int_{0}^{\infty} e^{-\rho t} U_{d}\left(Y_{t}\right) d X_{t}\right] \\
< & \infty .
\end{aligned}
$$

Applying Lemma 1 again yields:

$$
E\left[\int_{0}^{\infty} e^{-\rho t} X_{t}\left(u\left(C_{t}\right)\right)^{-} d t\right]<\infty .
$$

Thus, we obtain

$$
E\left[\int_{0}^{\infty} e^{-\rho t} X_{t}\left|u\left(C_{t}\right)\right| d t\right]<\infty
$$

It follows from Lemma 1 that

$$
E\left[\int_{0}^{\infty} e^{-\rho t} X_{t} u\left(C_{t}\right) d t\right]=X_{0} w+E\left[\int_{0}^{\infty} e^{-\rho t} U_{t}^{a}(C) d X_{t}\right]
$$

where $w=U_{0}^{a}(C)$.

Multiplying $e^{-r t}$ and taking expectations on both sides of (A.1), we obtain

$$
\begin{aligned}
U^{p}(y, C) & =E\left[\int_{0}^{\infty} e^{-r t}\left(Y_{t}-C_{t}\right) d t\right] \\
& \leq E\left[\int_{0}^{\infty} e^{-r t}\left(Y_{t}+\tilde{u}\left(e^{-(\rho-r) t} X_{t}\right)\right) d t\right]-E\left[\int_{0}^{\infty} e^{-\rho t} X_{t} u\left(C_{t}\right) d t\right] .
\end{aligned}
$$

Substituting (A.2) into (A.3) yields:

$$
\begin{aligned}
U^{p}(y, C) & \leq E\left[\int_{0}^{\infty} e^{-r t}\left(Y_{t}+\tilde{u}\left(e^{-(\rho-r) t} X_{t}\right)\right) d t\right]-X_{0} w-E\left[\int_{0}^{\infty} e^{-\rho t} U_{t}^{a}(C) d X_{t}\right] \\
& =L(y, x, X)-X_{0} w-E\left[\int_{0}^{\infty} e^{-\rho t}\left(U_{t}^{a}(C)-U_{d}\left(Y_{t}\right)\right) d X_{t}\right] \\
& \leq L(y, x, X)-x w,
\end{aligned}
$$

where the last inequality follows from the fact that $C \in \Gamma(y, w)$ and $X \in \mathcal{I}(x)$ and $X_{0}=x$. Equalities hold if and only if (21)-(22) hold. Q.E.D.

Proof of Theorem 2: First, we show that $C^{*}$ defined in the theorem satisfies the participation and promise-keeping constraints. Define $X^{\varepsilon} \equiv X^{*}+\varepsilon$ for $\varepsilon \in(0, \delta)$. The convexity of $\tilde{u}$ implies that

$$
e^{-(\rho-r) t} u\left(C_{t}^{*}\right) \leq \frac{\tilde{u}\left(X_{t}^{\varepsilon} e^{-(\rho-r) t}\right)-\tilde{u}\left(X_{t}^{*} e^{-(\rho-r) t}\right)}{\varepsilon} \leq \frac{\tilde{u}\left(X_{t}^{\delta} e^{-(\rho-r) t}\right)-\tilde{u}\left(X_{t}^{*} e^{-(\rho-r) t}\right)}{\delta} .
$$


By assumption, $E\left[\int_{0}^{\infty} e^{-r t}\left|\tilde{u}\left(X_{t}^{\delta} e^{-(\rho-r) t}\right)\right| d t\right]<\infty$. Furthermore,

$$
\begin{aligned}
E\left[\int_{0}^{\infty} e^{-\rho t}\left|u\left(C_{t}^{*}\right)\right| d t\right] & \leq E\left[\int_{0}^{\infty} e^{-r t} \frac{\left|X_{t}^{*} e^{-(\rho-r) t} u\left(C_{t}^{*}\right)\right|}{X_{0}^{*}} d t\right] \\
& \leq E\left[\int_{0}^{\infty} e^{-\rho t} \frac{\left|\tilde{u}\left(X_{t}^{*} e^{-(\rho-r) t}\right)\right|+C_{t}^{*}}{X_{0}^{*}} d t\right]<\infty
\end{aligned}
$$

It follows from $E\left[\int_{0}^{\infty} e^{-\rho t}\left|u\left(C_{t}^{*}\right)\right| d t\right]<\infty$ and $E\left[\int_{0}^{\infty} e^{-r t}\left|\tilde{u}\left(X_{t}^{\delta} e^{-(\rho-r) t}\right)\right| d t\right]<\infty$ that $E\left[\int_{0}^{\infty} e^{-\rho t}\left|\tilde{u}\left(X_{t}^{\varepsilon} e^{-(\rho-r) t}\right)\right| d t\right]<\infty$. Therefore, $X^{\varepsilon} \in \mathcal{I}\left(X_{0}^{*}+\varepsilon\right)$ and $\mathcal{L}\left(X^{\varepsilon}\right) \geq \mathcal{L}\left(X^{*}\right)$. This implies

$$
\lim _{\varepsilon \downarrow 0} \frac{\mathcal{L}\left(X^{\varepsilon}\right)-\mathcal{L}\left(X^{*}\right)}{\varepsilon} \geq 0
$$

or, equivalently,

$$
\lim _{\varepsilon \downarrow 0} E\left[\int_{0}^{\infty} e^{-r t} \frac{\tilde{u}\left(X_{t}^{\varepsilon} e^{-(\rho-r) t}\right)-\tilde{u}\left(X_{t}^{*} e^{-(\rho-r) t}\right)}{\varepsilon} d t\right]-w \geq 0 .
$$

By the Dominated Convergence Theorem,

$$
\begin{aligned}
& \lim _{\varepsilon \downarrow 0} E\left[\int_{0}^{\infty} e^{-r t} \frac{\tilde{u}\left(X_{t}^{\varepsilon} e^{-(\rho-r) t}\right)-\tilde{u}\left(X_{t}^{*} e^{-(\rho-r) t}\right)}{\varepsilon} d t\right] \\
= & E\left[\int_{0}^{\infty} e^{-r t} \lim _{\varepsilon \downarrow 0} \frac{\tilde{u}\left(X_{t}^{\varepsilon} e^{-(\rho-r) t}\right)-\tilde{u}\left(X_{t}^{*} e^{-(\rho-r) t}\right)}{\varepsilon} d t\right]=E\left[\int_{0}^{\infty} e^{-\rho t} u\left(C_{t}^{*}\right) d t\right] .
\end{aligned}
$$

It follows that

$$
U_{0}^{a}\left(C^{*}\right)=E\left[\int_{0}^{\infty} e^{-\rho t} u\left(C_{t}^{*}\right) d t\right] \geq w .
$$

Define $X^{\varepsilon}(\omega, s)=X^{*}(\omega, s)+\varepsilon \mathbf{1}_{A \times[t, \infty)}(\omega, s)$ for $\varepsilon>0, t>0$ and $A \in \mathcal{F}_{t}$, where $\mathbf{1}$ denotes an indicator function. A similar argument shows that $X^{\varepsilon} \in \mathcal{I}\left(X_{0}^{*}\right)$. Since $\mathcal{L}\left(X^{\varepsilon}\right) \geq \mathcal{L}\left(X^{*}\right)$, we obtain

$$
\lim _{\varepsilon \downarrow 0} \frac{\mathcal{L}\left(X^{\varepsilon}\right)-\mathcal{L}\left(X^{*}\right)}{\varepsilon} \geq 0 .
$$

By a similar argument, we can show that

$$
\begin{aligned}
& \lim _{\varepsilon \downarrow 0} \frac{\mathcal{L}\left(X^{\varepsilon}\right)-\mathcal{L}\left(X^{*}\right)}{\varepsilon} \\
= & \lim _{\varepsilon \downarrow 0} E\left[\int_{0}^{\infty} e^{-r t} \frac{\tilde{u}\left(X_{t}^{\varepsilon} e^{-(\rho-r) t}\right)-\tilde{u}\left(X_{t}^{*} e^{-(\rho-r) t}\right)}{\varepsilon} d t\right]-E\left[1_{A} e^{-\rho t} U_{d}\left(Y_{t}\right)\right] \\
= & E\left[\mathbf{1}_{A} \int_{t}^{\infty} e^{-\rho s} u\left(C_{s}^{*}\right) d s\right]-E\left[\mathbf{1}_{A} e^{-\rho t} U_{d}\left(Y_{t}\right)\right] \geq 0 .
\end{aligned}
$$

Because $A$ is an arbitrary subset in $\mathcal{F}_{t}$, it follows that

$$
E_{t}\left[\int_{t}^{\infty} e^{-\rho s} u\left(C_{s}^{*}\right) d s\right] \geq e^{-\rho t} U_{d}\left(Y_{t}\right)
$$


or, equivalently,

$$
E_{t}\left[\int_{t}^{\infty} e^{-\rho(s-t)} u\left(C_{s}^{*}\right) d s\right] \geq U_{d}\left(Y_{t}\right)
$$

Multiplying $e^{-\rho t}$, integrating with respect to $X$, and taking expectations on both sides of the above inequality, we can derive that

$$
E\left[\int_{0}^{\infty} e^{-\rho t} U_{t}^{a}\left(C^{*}\right) d X_{t}\right] \geq E\left[\int_{0}^{\infty} e^{-\rho t} U_{d}\left(Y_{t}\right) d X_{t}\right] .
$$

Second, we show that (A.4) and (A.5) must hold with equality. To prove this, consider $\bar{X}^{\varepsilon}=X^{*}(1+\varepsilon)$ for $\varepsilon \in(-\delta, \delta)$. The convexity of $\tilde{u}$ implies that

$$
\begin{aligned}
\frac{\tilde{u}\left(\bar{X}_{t}^{-\delta} e^{-(\rho-r) t}\right)-\tilde{u}\left(X_{t}^{*} e^{-(\rho-r) t}\right)}{-\delta} & \leq \frac{\tilde{u}\left(\bar{X}_{t}^{\varepsilon} e^{-(\rho-r) t}\right)-\tilde{u}\left(X_{t}^{*} e^{-(\rho-r) t}\right)}{\varepsilon} \\
& \leq \frac{\tilde{u}\left(\bar{X}_{t}^{\delta} e^{-(\rho-r) t}\right)-\tilde{u}\left(X_{t}^{*} e^{-(\rho-r) t}\right)}{\delta}
\end{aligned}
$$

which implies $\bar{X}^{\varepsilon} \in \mathcal{I}\left(X_{0}^{*}(1+\varepsilon)\right)$. Since $\mathcal{L}\left(\bar{X}^{\varepsilon}\right) \geq \mathcal{L}\left(X^{*}\right)$, we obtain

$$
\lim _{\varepsilon \downarrow 0} \frac{\mathcal{L}\left(\bar{X}^{\varepsilon}\right)-\mathcal{L}\left(X^{*}\right)}{\varepsilon} \geq 0 \text { and } \lim _{\varepsilon \uparrow 0} \frac{\mathcal{L}\left(\bar{X}^{\varepsilon}\right)-\mathcal{L}\left(X^{*}\right)}{\varepsilon} \leq 0 .
$$

By the Dominated Convergence Theorem,

$$
\lim _{\varepsilon \rightarrow 0} \frac{\mathcal{L}\left(\bar{X}^{\varepsilon}\right)-\mathcal{L}\left(X^{*}\right)}{\varepsilon}=E\left[\int_{0}^{\infty} e^{-\rho t} X_{t}^{*} u\left(C_{t}^{*}\right) d t\right]-E\left[\int_{0}^{\infty} e^{-\rho t} U_{d}\left(Y_{t}\right) d X_{t}^{*}\right]-X_{0}^{*} w,
$$

which, according to (A.6), should be both nonnegative and nonpositive. It follows that

$$
E\left[\int_{0}^{\infty} e^{-\rho t} X_{t}^{*} u\left(C_{t}^{*}\right) d t\right]-E\left[\int_{0}^{\infty} e^{-\rho t} U_{d}\left(Y_{t}\right) d X_{t}^{*}\right]-X_{0}^{*} w=0 .
$$

By Lemma 1,

$$
E\left[\int_{0}^{\infty} e^{-\rho t} X_{t}^{*} u\left(C_{t}^{*}\right) d t\right]=X_{0}^{*} U_{0}^{a}\left(C^{*}\right)+E\left[\int_{0}^{\infty} e^{-\rho t} U_{t}^{a}\left(C^{*}\right) d X_{t}^{*}\right]
$$

Plugging this equation into (A.7) yields:

$$
X_{0}^{*}\left(U_{0}^{a}\left(C^{*}\right)-w\right)+E\left[\int_{0}^{\infty} e^{-\rho t}\left(U_{t}^{a}\left(C^{*}\right)-U_{d}\left(Y_{t}\right)\right) d X_{t}^{*}\right]=0
$$

Thus, (A.4) and (A.5) must hold with equality since $X_{0}^{*}>0$.

Finally, we show that $C^{*}$ is optimal in the primal problem. By Theorem 1,

$$
U^{p}\left(y, C^{*}\right) \leq \sup _{C \in \Gamma(y, w)} U^{p}(y, C) \leq \inf _{X \in \mathcal{I}(x), x>0} L(y, x, X)-x w \leq L\left(y, x^{*}, X^{*}\right)-x^{*} w .
$$

Since $C^{*}$ and $X^{*}$ satisfy (21)-(22), it follows from Theorem 1 that all the inequalities in (A.8) must hold with equalities, and hence $C^{*}$ is in fact the optimal solution to the primal problem. In addition,

as desired. Q.E.D.

$$
V(y, w)=\inf _{x>0} \tilde{V}(y, x)-x w
$$


Proof of Theorem 3: Step 1. Define a process

$$
G_{t}^{X}=\int_{0}^{t} e^{-r s}\left(Y_{s}+\tilde{u}\left(Z_{s}\right)\right) d s-\int_{0}^{t} e^{-\rho s} U_{d}\left(Y_{s}\right) d X_{s}+e^{-r t} J\left(Y_{t}, Z_{t}\right),
$$

for any $X \in \mathcal{I}(z)$ and $Z_{t}=e^{-(\rho-r) t} X_{t}$ with $X_{0}=Z_{0}=z$. We show that $G_{t}^{X}$ is a submartingale.

By the generalized Ito's Lemma (e.g., Harrison (1985)),

$$
\begin{aligned}
e^{r t} d G_{t}^{X}= & \left(Y_{t}+\tilde{u}\left(Z_{t}\right)\right) d t-e^{(r-\rho) t} U_{d}\left(Y_{t}\right) d X_{t}+d J\left(Y_{t}, Z_{t}\right)-r J\left(Y_{t}, Z_{t}\right) d t \\
= & \left(Y_{t}+\tilde{u}\left(Z_{t}\right)\right) d t-e^{(r-\rho) t} U_{d}\left(Y_{t}\right) d X_{t}^{c}+J_{y}\left(Y_{t}, Z_{t}\right) \mu\left(Y_{t}\right) d t \\
& +J_{y}\left(Y_{t}, Z_{t}\right) \sigma\left(Y_{t}\right) d B_{t}+\frac{1}{2} J_{y y}\left(Y_{t}, Z_{t}\right) \sigma^{2}\left(Y_{t}\right) d t \\
& +J_{z}\left(Y_{t}, Z_{t}\right)\left(e^{(r-\rho) t} d X_{t}^{c}-(\rho-r) Z_{t} d t\right)-r J\left(Y_{t}, Z_{t}\right) d t \\
& +J\left(Y_{t}, Z_{t}\right)-J\left(Y_{t}, Z_{t-}\right)-e^{(r-\rho) t} U_{d}\left(Y_{t}\right) \Delta X_{t} \\
= & \left(Y_{t}+\tilde{u}\left(Z_{t}\right)\right) d t-(\rho-r) Z_{t} J_{z}\left(Y_{t}, Z_{t}\right) d t+J_{y}\left(Y_{t}, Z_{t}\right) \mu\left(Y_{t}\right) d t \\
& +\frac{1}{2} J_{y y}\left(Y_{t}, Z_{t}\right) \sigma^{2}\left(Y_{t}\right) d t-r J\left(Y_{t}, Z_{t}\right) d t \\
& +e^{(r-\rho) t}\left(J_{z}\left(Y_{t}, Z_{t}\right)-U_{d}\left(Y_{t}\right)\right) d X_{t}^{c}+J_{y}\left(Y_{t}, Z_{t}\right) \sigma\left(Y_{t}\right) d B_{t} \\
& +J\left(Y_{t}, Z_{t}\right)-J\left(Y_{t}, Z_{t-}\right)-e^{(r-\rho) t} U_{d}\left(Y_{t}\right) \Delta X_{t},
\end{aligned}
$$

where $\Delta X_{t} \equiv X_{t}-X_{t-}$ and $X^{c}$ is the continuous part of $X$. Thus, for $T \geq t$,

$$
\begin{aligned}
G_{T}^{X}= & G_{t}^{X}+\underbrace{\int_{t}^{T} e^{-r s}\left(Y_{s}+\tilde{u}\left(Z_{s}\right)+\mathcal{A} J\left(Y_{s}, Z_{s}\right)\right) d s}_{I_{1}(T)} \\
& +\underbrace{\int_{t}^{T} e^{-\rho s}\left(J_{z}\left(Y_{s}, Z_{s}\right)-U_{d}\left(Y_{s}\right)\right) d X_{s}^{c}}_{I_{2}(T)}+\underbrace{\int_{t}^{T} e^{-r s} J_{y}\left(Y_{s}, Z_{s}\right) \sigma\left(Y_{s}\right) d B_{s}}_{I_{3}(T)} \\
& +\underbrace{\sum_{t \leq s \leq T} e^{-r s}\left(J\left(Y_{s}, Z_{s}\right)-J\left(Y_{s}, Z_{s-}\right)\right.}_{\Sigma(T)}-e^{\left.e^{(r-\rho) s} U_{d}\left(Y_{s}\right) \Delta X_{s}\right)} .
\end{aligned}
$$

Taking expectations conditional on the information at time $t$, we obtain

$$
E_{t}\left[G_{T}^{X}\right]=G_{t}^{X}+E_{t}\left[I_{1}(T)\right]+E_{t}\left[I_{2}(T)\right]+E_{t}\left[I_{3}(T)\right]+E_{t}[\Sigma(T)]
$$

By the variational inequality and $d X_{t}^{c} \geq 0$, we can show that $E_{t}\left[I_{1}(T)\right] \geq 0$ and $E_{t}\left[I_{2}(T)\right] \geq 0$. By condition (28), $E_{t}\left[I_{3}(T)\right]=0$. Using the variational inequality, we can also show that

$$
J\left(Y_{s}, Z_{s}\right)-J\left(Y_{s}, Z_{s-}\right)-e^{(r-\rho) s} U_{d}\left(Y_{s}\right) \Delta X_{s}=\int_{Z_{s}-\Delta Z_{s}}^{Z_{s}}\left(J_{z}\left(Y_{s}, z\right)-U_{d}\left(Y_{s}\right)\right) d z \geq 0,
$$


where $\Delta Z_{s}=e^{(r-\rho) s} \Delta X_{s}$. Thus, $E_{t}[\Sigma(T)] \geq 0$. It follows that $\left\{G_{t}^{X}\right\}_{t \geq 0}$ is a submartingale. This implies that

$$
J\left(Y_{0}, Z_{0}\right)=G_{0}^{X} \leq E\left[G_{t}^{X}\right],
$$

for all $t$. Taking limits and using $\lim _{t \rightarrow \infty} E\left[e^{-r t} J\left(Y_{t}, Z_{t}\right)\right]=0$, we have

$$
J\left(Y_{0}, Z_{0}\right) \leq E\left[\int_{0}^{\infty} e^{-r t}\left(Y_{t}+\tilde{u}\left(Z_{t}\right)\right) d t-\int_{0}^{\infty} e^{-\rho t} U_{d}\left(Y_{t}\right) d X_{t}\right] .
$$

Step 2. Show that $\left\{G_{t}^{X^{*}}\right\}_{t \geq 0}$ is a martingale. Note that $X^{*}$ is continuous (e.g., Harrison and Taksar (1983) and Harrison (1985)). We can use assumptions in the theorem to verify $E_{t}\left[I_{1}(T)\right]=E_{t}\left[I_{2}(T)\right]=E_{t}[\Sigma(T)]=0$ for $X^{*}$.

The above two steps imply that

$$
J(y, z)=\inf _{X \in \mathcal{I}(z)} E\left[\int_{0}^{\infty} e^{-r t}\left(Y_{t}+\tilde{u}\left(Z_{t}\right)\right) d t\right]-E\left[\int_{0}^{\infty} e^{-\rho t} U_{d}\left(Y_{t}\right) d X_{t}\right],
$$

and $X^{*}$ attains the minimum. By assumption, $J(y, z)$ is strictly convex in $z$ and $J_{z}\left(y, z^{*}\right)=w$. Thus, $z^{*}$ achieves the minimum of $J(y, z)$. This implies that $X^{*}$ with $X_{0}^{*}=z^{*}$ solves the dual problem (14).

Step 3. Show that $\left\{C_{t}^{*}\right\}_{t \geq 0}$ is optimal. This follows from Theorem 2. The proof of the remaining results is trivial. Q.E.D.

Proof of Theorem 4: By the integrability condition (53),

$$
E\left[\int_{0}^{\infty} e^{-\rho t}\left(1+H_{t}\right)\left(u^{p}\left(A\left(Y_{t}\right)-C_{t}\right)\right)^{+} d t\right]<E\left[\int_{0}^{\infty} e^{-\rho t}\left(1+H_{t}\right)\left(u^{p}\left(A\left(Y_{t}\right)\right)\right)^{+} d t\right]<\infty .
$$

By the principal's participation constraint (44) and an argument similar to that in the proof of Theorem 1, we can show that

$$
E\left[\int_{0}^{\infty} e^{-\rho t}\left(1+H_{t}\right)\left(u^{p}\left(A\left(Y_{t}\right)-C_{t}\right)\right)^{-} d t\right]<\infty .
$$

Thus,

$$
E\left[\int_{0}^{\infty} e^{-\rho t}\left|u^{p}\left(A\left(Y_{t}\right)-C_{t}\right)\right| d t\right] \leq E\left[\int_{0}^{\infty} e^{-\rho t}\left(1+H_{t}\right)\left|u^{p}\left(A\left(Y_{t}\right)-C_{t}\right)\right| d t\right]<\infty .
$$

It follows from Lemma 1 that

$$
E\left[\int_{0}^{\infty} e^{-\rho t} H_{t} u^{p}\left(A\left(Y_{t}\right)-C_{t}\right) d t\right]=H_{0} U^{p}(y, C)+E\left[\int_{0}^{\infty} e^{-\rho t} U_{t}^{p}\left(A\left(Y_{t}\right)-C\right) d H_{t}\right],
$$

where $H_{0}=h$. 
By the definition of $\tilde{u}$ in (48),

$$
u^{p}\left(A\left(Y_{t}\right)-C_{t}\right) \leq \tilde{u}\left(Y_{t}, X_{t}, H_{t}\right)-H_{t} u^{p}\left(A\left(Y_{t}\right)-C_{t}\right)-X_{t} u\left(C_{t}\right),
$$

for any $C \in \Phi(y, w)$. It follows that

$$
X_{t} u\left(C_{t}\right) \leq \tilde{u}\left(Y_{t}, X_{t}, H_{t}\right)-\left(1+H_{t}\right) u^{p}\left(A\left(Y_{t}\right)-C_{t}\right)
$$

Since each term on the right-hand side of the above inequality is integrable, we deduce that

$$
E\left[\int_{0}^{\infty} e^{-\rho t} X_{t}\left(u\left(C_{t}\right)\right)^{+} d t\right]<\infty
$$

By an argument similar to that in the proof of Theorem 1, we can derive (A.2).

Multiplying $e^{-\rho t}$ and taking expectations on both sides of (A.10), we obtain

$$
\begin{aligned}
U^{p}(y, C) \leq & E\left[\int_{0}^{\infty} e^{-\rho t}\left(\tilde{u}\left(Y_{t}, X_{t}, H_{t}\right)-H_{t} u^{p}\left(A\left(Y_{t}\right)-C_{t}\right)-X_{t} u\left(C_{t}\right)\right) d t\right] \\
= & L(y, x, h, X, H)-E\left[\int_{0}^{\infty} e^{-\rho t} H_{t} u^{p}\left(A\left(Y_{t}\right)-C_{t}\right) d t\right]-E\left[\int_{0}^{\infty} e^{-\rho t} X_{t} u\left(C_{t}\right) d t\right] \\
& +E\left[\int_{0}^{\infty} e^{-\rho t} U_{d}\left(Y_{t}\right) d X_{t}\right]+E\left[\int_{0}^{\infty} e^{-\rho t} U_{d}^{p}\left(A\left(Y_{t}\right)-Y_{t}\right) d H_{t}\right] .
\end{aligned}
$$

Plugging (A.2) and (A.9) into (A.11), we obtain

$$
\begin{aligned}
U^{p}(y, C) \leq & L(y, x, h, X, H)-x w-E\left[\int_{0}^{\infty} e^{-\rho t} U_{t}^{a}(C) d X_{t}\right] \\
& -h U^{p}(y, C)-E\left[\int_{0}^{\infty} e^{-\rho t} U_{t}^{p}(A(Y)-C) d H_{t}\right] \\
& +E\left[\int_{0}^{\infty} e^{-\rho t} U_{d}\left(Y_{t}\right) d X_{t}\right]+E\left[\int_{0}^{\infty} e^{-\rho t} U_{d}^{p}\left(A\left(Y_{t}\right)-Y_{t}\right) d H_{t}\right] \\
= & L(y, x, h, X, H)-x w-h U^{p}(y, C)-E\left[\int_{0}^{\infty} e^{-\rho t}\left(U_{t}^{a}(C)-U_{d}\left(Y_{t}\right)\right) d X_{t}\right] \\
& -E\left[\int_{0}^{\infty} e^{-\rho t}\left(U_{t}^{p}(A(Y)-C)-U_{d}^{p}\left(A\left(Y_{t}\right)-Y_{t}\right)\right) d H_{t}\right] \\
\leq & L(y, x, h, X, H)-x w-h U^{p}(y, C),
\end{aligned}
$$

where the last inequality follows from the fact that $C \in \Phi(y, w)$. Thus, we obtain (57). Equalities hold if and only if (21)-(22) hold. Q.E.D.

Proof of Theorem 5: Because the idea for this proof is similar to that for Theorem 2, we shall sketch the main steps only. First, we show that $C^{*}$ defined in the theorem is sustainable and satisfies the participation constraint. Define $X^{\varepsilon} \equiv X^{*}+\varepsilon$ for $\varepsilon \in(0, \delta)$. The convexity of $\tilde{u}$ 
implies $E\left[\int_{0}^{\infty} e^{-\rho t}\left|\tilde{u}\left(X_{t}^{\varepsilon}, H_{t}^{*}, Y_{t}\right)\right| d t\right]<\infty$. Define $\mathcal{L}\left(X^{\varepsilon}, H^{*}\right)$ as in (49). Since $\mathcal{L}\left(X^{\varepsilon}, H^{*}\right) \geq$ $\mathcal{L}\left(X^{*}, H^{*}\right)$, we obtain

$$
\lim _{\varepsilon \downarrow 0} \frac{\mathcal{L}\left(X^{\varepsilon}, H^{*}\right)-\mathcal{L}\left(X^{*}, H^{*}\right)}{\varepsilon} \geq 0 .
$$

By the Dominated Convergence Theorem and a similar argument in the proof of Theorem 2, we can show that

$$
U_{0}^{a}\left(C^{*}\right)=E\left[\int_{0}^{\infty} e^{-\rho t} u\left(C_{t}^{*}\right)\right] \geq w
$$

Define $X^{\varepsilon}=X^{*}+\varepsilon \mathbf{1}_{A \times[t, \infty)}$ for $\varepsilon>0, t>0$ and $A \in \mathcal{F}_{t}$. By a similar argument in the proof of Theorem 2,

$$
\lim _{\varepsilon \downarrow 0} \frac{\mathcal{L}\left(X^{\varepsilon}, H^{*}\right)-\mathcal{L}\left(X^{*}, H^{*}\right)}{\varepsilon}=E\left[\mathbf{1}_{A} \int_{t}^{\infty} e^{-\rho s} u\left(C_{s}^{*}\right) d s\right]-E\left[\mathbf{1}_{A} e^{-\rho t} U_{d}\left(Y_{t}\right)\right] \geq 0 .
$$

Because $A$ is an arbitrary subset in $\mathcal{F}_{t}$, it follows that

$$
U_{t}^{a}\left(C^{*}\right)=E_{t}\left[\int_{t}^{\infty} e^{-\rho(s-t)} u\left(C_{s}^{*}\right) d s\right] \geq U_{d}\left(Y_{t}\right)
$$

Multiplying $e^{-\rho t}$, integrating with respect to $X$, and taking expectations on both sides of the inequality, we can derive that

$$
E\left[\int_{0}^{\infty} e^{-\rho t} U_{t}^{a}\left(C^{*}\right) d X_{t}\right] \geq E\left[\int_{0}^{\infty} e^{-\rho t} U_{d}\left(Y_{t}\right) d X_{t}\right]
$$

Similarly, define $H^{\varepsilon}=H^{*}+\varepsilon \mathbf{1}_{A \times[t, \infty)}$ for $\varepsilon>0, t>0$ and $A \in \mathcal{F}_{t}$. By a similar argument, we can show that

$$
U_{t}^{p}\left(A(Y)-C^{*}\right) \geq U_{d}^{p}\left(A\left(Y_{t}\right)-Y_{t}\right)
$$

and

$$
E\left[\int_{0}^{\infty} e^{-\rho t} U_{t}^{p}\left(A(Y)-C^{*}\right) d H_{t}\right] \geq E\left[\int_{0}^{\infty} e^{-\rho t} U_{d}^{p}\left(A\left(Y_{t}\right)-Y_{t}\right) d H_{t}\right] .
$$

Second, we show below that (A.12), (A.14), and (A.16) must hold with equality. To prove this, consider $\bar{X}^{\varepsilon}=X^{*}(1+\varepsilon)$ for small $\varepsilon \in(-\delta, \delta)$. Since $\mathcal{L}\left(\bar{X}^{\varepsilon}, H^{*}\right) \geq \mathcal{L}\left(X^{*}, H^{*}\right)$, we obtain

$$
\lim _{\varepsilon \downarrow 0} \frac{\mathcal{L}\left(\bar{X}^{\varepsilon}, H^{*}\right)-\mathcal{L}\left(X^{*}, H^{*}\right)}{\varepsilon} \geq 0 \text { and } \lim _{\varepsilon \uparrow 0} \frac{\mathcal{L}\left(\bar{X}^{\varepsilon}, H^{*}\right)-\mathcal{L}\left(X^{*}, H^{*}\right)}{\varepsilon} \leq 0 .
$$

By the Dominated Convergence Theorem,

$$
E\left[\int_{0}^{\infty} e^{-\rho t} X_{t}^{*} u\left(C_{t}^{*}\right) d t\right]-E\left[\int_{0}^{\infty} e^{-\rho t} U_{d}\left(Y_{t}\right) d X_{t}^{*}\right]-X_{0}^{*} w=0 .
$$

Since

$$
E\left[\int_{0}^{\infty} e^{-\rho t} X_{t}^{*} u\left(C_{t}^{*}\right) d t\right]=X_{0}^{*} U_{0}^{a}\left(C^{*}\right)+E\left[\int_{0}^{\infty} e^{-\rho t} U_{t}^{a}\left(C^{*}\right) d X_{t}^{*}\right],
$$


we obtain

$$
X_{0}^{*}\left(U_{0}^{a}\left(C^{*}\right)-w\right)+E\left[\int_{0}^{\infty} e^{-\rho t}\left(U_{t}^{a}\left(C^{*}\right)-U_{d}\left(Y_{t}\right)\right) d X_{t}^{*}\right]=0 .
$$

Thus, (A.12) and (A.14) must hold with equality. Similarly, we can define $\bar{H}^{\varepsilon}=H^{*}(1+\varepsilon)$ and use a similar argument to show that (A.16) holds with equality.

Finally, we show that $C^{*}$ is optimal in the primal problem. Since $C^{*} \in \Phi(y, w)$, it follows from Theorem 4 that

$$
\begin{aligned}
U^{p}\left(y, C^{*}\right) & \leq \sup _{C \in \Phi(y, w)} U^{p}(y, C) \leq \inf _{X \in \mathcal{I}(x), H \in \mathcal{I}(h), x>0, h \geq 0} \frac{L(y, x, h, X, H)}{1+h}-\frac{x}{1+h} w \\
& =\inf _{x>0, h \geq 0} \frac{\tilde{V}(y, x, h)}{1+h}-\frac{x}{1+h} w \\
& =\inf _{z>0} \tilde{V}(y, z, 0)-z w=\inf _{X \in \mathcal{I}, H \in \mathcal{I}(0)} \mathcal{L}(X, H) \\
& =L\left(y, x^{*}, 0, X^{*}, H^{*}\right)-x^{*} w,
\end{aligned}
$$

where the first equality in the third line follows from the linear homogeneity of $\tilde{V}$ in $(x, 1+h)$ and the change of variables $z=x /(1+h)$. By Theorem 4 , all inequalities hold with equalities. Thus, $C^{*}$ is the optimal solution to the primal problem. In addition,

$$
V(y, w)=\inf _{z>0} \tilde{V}(y, z, 0)-z w=\inf _{x>0, h \geq 0} \frac{\tilde{V}(y, x, h)}{1+h}-\frac{x}{1+h} w,
$$

as desired. Q.E.D.

Proof of Proposition 2: By the value-matching and super-contact conditions, we can derive a system of four nonlinear equations for four unknowns $\left(b_{1}, b_{2}, A_{1}, A_{2}\right)$ :

$$
\begin{aligned}
\frac{1}{\rho \alpha} b_{1}^{\frac{\alpha}{1-\alpha}}+A_{1}\left(1-\beta_{1}\right) b_{1}^{\frac{\alpha-\beta_{1}}{1-\alpha}}+A_{2}\left(1-\beta_{2}\right) b_{1}^{\frac{\alpha-\beta_{2}}{1-\alpha}}=\kappa, \\
\frac{1}{\rho}+A_{1}\left(1-\beta_{1}\right)\left(\alpha-\beta_{1}\right) b_{1}^{\frac{-\beta_{1}}{1-\alpha}}+A_{2}\left(1-\beta_{2}\right)\left(\alpha-\beta_{2}\right) b_{1}^{\frac{-\beta_{2}}{1-\alpha}}=0, \\
\frac{1}{\rho-\mu}-\frac{b_{2}^{\frac{1}{1-\alpha}}}{\rho}+A_{1}\left(\beta_{1}-\alpha\right) b_{2}^{\frac{1-\beta_{1}}{1-\alpha}}+A_{2}\left(\beta_{2}-\alpha\right) b_{2}^{\frac{1-\beta_{2}}{1-\alpha}}=0, \\
\frac{1}{\rho}+A_{1}\left(1-\beta_{1}\right)\left(\alpha-\beta_{1}\right) b_{2}^{\frac{-\beta_{1}}{1-\alpha}}+A_{2}\left(1-\beta_{2}\right)\left(\alpha-\beta_{2}\right) b_{2}^{\frac{-\beta_{2}}{1-\alpha}}=0 .
\end{aligned}
$$

The proof of the proposition contains five steps.

Step 1. We can solve for $A_{1}$ and $A_{2}$ for any $b_{2}$ using (A.19) and (A.20) as follows. (Figure 8 plots $A_{1}\left(b_{2}\right)$ and $A_{2}\left(b_{2}\right)$.) Plugging (A.20) into (A.19), we rewrite (A.19) and (A.20) as

$$
\begin{aligned}
\frac{1}{\rho-\mu}+A_{1} \beta_{1}\left(\beta_{1}-\alpha\right) b_{2}^{\frac{1-\beta_{1}}{1-\alpha}}+A_{2} \beta_{2}\left(\beta_{2}-\alpha\right) b_{2}^{\frac{1-\beta_{2}}{1-\alpha}} & =0, \\
\frac{1}{\rho}+A_{1}\left(\beta_{1}-1\right)\left(\beta_{1}-\alpha\right) b_{2}^{-\frac{\beta_{1}}{1-\alpha}}+A_{2}\left(\beta_{2}-1\right)\left(\beta_{2}-\alpha\right) b_{2}^{-\frac{\beta_{2}}{1-\alpha}} & =0 .
\end{aligned}
$$


This linear system of equations in $\left(A_{1}, A_{2}\right)$ gives

$$
\begin{aligned}
& \left(\begin{array}{c}
A_{1} \\
A_{2}
\end{array}\right)=\left(\begin{array}{cc}
\beta_{1}\left(\beta_{1}-\alpha\right) b_{2}^{\frac{1-\beta_{1}}{1-\alpha}} & \beta_{2}\left(\beta_{2}-\alpha\right) b_{2}^{\frac{1-\beta_{2}}{1-\alpha}} \\
\left(\beta_{1}-1\right)\left(\beta_{1}-\alpha\right) b_{2}^{-\frac{\beta_{1}}{1-\alpha}} & \left(\beta_{2}-1\right)\left(\beta_{2}-\alpha\right) b_{2}^{-\frac{\beta_{2}}{1-\alpha}}
\end{array}\right)^{-1}\left(\begin{array}{c}
-\frac{1}{\rho-\mu} \\
-\frac{1}{\rho}
\end{array}\right) \\
& =\left(\begin{array}{c}
\rho\left(\beta_{2}-1\right)\left(\beta_{2}-\alpha\right) b_{2}^{\frac{\beta_{1}-1}{1-\alpha}}-(\rho-\mu) \beta_{2}\left(\beta_{2}-\alpha\right) b_{2}^{\frac{\beta_{1}}{1-\alpha}} \\
-\rho\left(\beta_{1}-1\right)\left(\beta_{1}-\alpha\right) b_{2}^{\frac{\beta_{2}-1}{1-\alpha}}+(\rho-\mu) \beta_{1}\left(\beta_{1}-\alpha\right) b_{2}^{\frac{\beta_{2}}{1-\alpha}}
\end{array}\right) m^{-1},
\end{aligned}
$$

where $m \equiv-\left(\beta_{1}-\alpha\right)\left(\beta_{2}-\alpha\right)\left(\beta_{2}-\beta_{1}\right) \rho(\rho-\mu)>0$. We show some properties of $\left(A_{1}\left(b_{2}\right), A_{2}\left(b_{2}\right)\right)$ to be used later. First, $A_{1}<0$ if $b_{2}>1$. Because $\beta_{2}>1>\alpha$, it is sufficient to verify that

$$
\rho\left(\beta_{2}-1\right)-(\rho-\mu) \beta_{2} b_{2}^{\frac{1}{1-\alpha}} \leq \rho\left(\beta_{2}-1\right)-(\rho-\mu) \beta_{2}=\mu \beta_{2}-\rho=-\frac{\sigma^{2}}{2} \beta_{2}\left(\beta_{2}-1\right)<0 .
$$

Second, $A_{2}<0$ for $b_{2} \in(1, \bar{b})$, where $\bar{b} \equiv\left(\frac{\rho}{\rho-\mu} \frac{1-\beta_{1}}{-\beta_{1}}\right)^{1-\alpha}$. Because $\beta_{1}<\alpha$, it is sufficient to verify that

$$
-\rho\left(1-\beta_{1}\right)+(\rho-\mu)\left(-\beta_{1}\right) b_{2}^{\frac{1}{1-\alpha}}<0
$$

which follows from $b_{2}<\bar{b}$. Third, both $A_{1}$ and $A_{2}$ increase in $b_{2}>1$. The sign of $A_{1}^{\prime}\left(b_{2}\right)$ is the same as the sign of $\rho\left(\beta_{2}-1\right)\left(\beta_{1}-1\right)-(\rho-\mu) \beta_{2} \beta_{1} b_{2}^{\frac{1}{1-\alpha}}$, which is negative because

$$
\begin{aligned}
\rho\left(\beta_{2}-1\right)\left(\beta_{1}-1\right)-(\rho-\mu) \beta_{2} \beta_{1} b_{2}^{\frac{1}{1-\alpha}} & <\rho\left(\beta_{2}-1\right)\left(\beta_{1}-1\right)-(\rho-\mu) \beta_{2} \beta_{1} \\
& =\rho+\mu \beta_{2} \beta_{1}-\rho\left(\beta_{2}+\beta_{1}\right) \\
& =\rho-\mu \frac{\rho}{1 / 2 \sigma^{2}}+\rho\left(\frac{\mu}{1 / 2 \sigma^{2}}-1\right)=0 .
\end{aligned}
$$

Using the same steps, we can verify that $A_{2}^{\prime}\left(b_{2}\right)<0$.

Step 2. We show that there is a unique solution $b_{1} \in(0,1)$ to equation (A.18) for any $b_{2} \in(1, \bar{b})$ and $\left(A_{1}\left(b_{2}\right)<0, A_{2}\left(b_{2}\right)<0\right)$. First, when $A_{1}$ and $A_{2}$ are fixed, then the function

$$
f\left(A_{1}, A_{2}, b\right) \equiv \frac{1}{\rho}+A_{1}\left(1-\beta_{1}\right)\left(\alpha-\beta_{1}\right) b^{\frac{-\beta_{1}}{1-\alpha}}+A_{2}\left(1-\beta_{2}\right)\left(\alpha-\beta_{2}\right) b^{\frac{-\beta_{2}}{1-\alpha}}
$$

is single-peaked in $b$ because

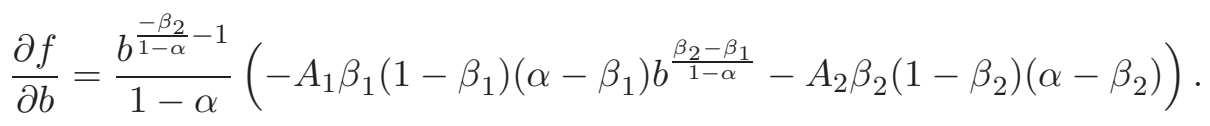

Because $A_{1}<0$ and $\beta_{1}<0$, the sign of $\frac{\partial f}{\partial b}$ turns from positive to negative only once. That $f$ is single-peaked in $b$ implies that $f\left(A_{1}, A_{2}, b\right)=0$ pins down two solutions, one of which is $b_{2}$ (as 

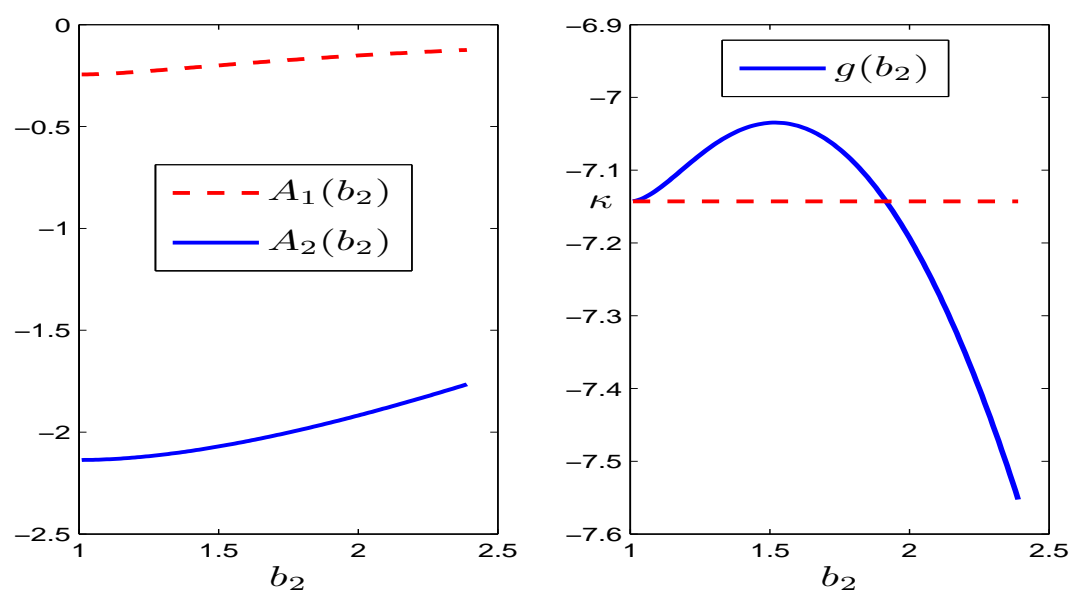

Figure 8: The functions $A_{1}\left(b_{2}\right), A_{2}\left(b_{2}\right)$, and $g\left(b_{2}\right)$.

we already see in equation (A.20)). Second, we verify that $b_{2}$ is on the downside of $f$, which would imply that $b_{1}$ is on the upside of $f$. We have

$$
\begin{aligned}
& \left.\frac{\partial f\left(A_{1}, A_{2}, b_{2}\right)}{\partial b}\right|_{A_{1}=A_{1}\left(b_{2}\right), A_{2}=A_{2}\left(b_{2}\right)} \\
= & \frac{1}{1-\alpha}\left(A_{1} \beta_{1}\left(\beta_{1}-1\right)\left(\alpha-\beta_{1}\right) b_{2}^{\frac{-\beta_{1}}{1-\alpha}-1}+A_{2} \beta_{2}\left(\beta_{2}-1\right)\left(\alpha-\beta_{2}\right) b_{2}^{\frac{-\beta_{2}}{1-\alpha}-1}\right) \\
= & \frac{1}{m(1-\alpha)}\left(\left(\rho\left(\beta_{2}-1\right)\left(\beta_{2}-\alpha\right) b_{2}^{\frac{\beta_{1}-1}{1-\alpha}}-(\rho-\mu) \beta_{2}\left(\beta_{2}-\alpha\right) b_{2}^{\frac{\beta_{1}}{1-\alpha}}\right) \beta_{1}\left(\beta_{1}-1\right)\left(\alpha-\beta_{1}\right) b_{2}^{\frac{-\beta_{1}}{1-\alpha}-1}\right. \\
& \left.+\left(-\rho\left(\beta_{1}-1\right)\left(\beta_{1}-\alpha\right) b_{2}^{\frac{\beta_{2}-1}{1-\alpha}}+(\rho-\mu) \beta_{1}\left(\beta_{1}-\alpha\right) b_{2}^{\frac{\beta_{2}}{1-\alpha}}\right) \beta_{2}\left(\beta_{2}-1\right)\left(\alpha-\beta_{2}\right) b_{2}^{\frac{-\beta_{2}}{1-\alpha}-1}\right) \\
= & \frac{b_{2}^{\frac{\alpha-2}{1-\alpha}}\left(\beta_{2}-\alpha\right)\left(\beta_{1}-\alpha\right)}{m(1-\alpha)}\left(-\rho\left(\beta_{2}-1\right)\left(\beta_{1}-1\right) \beta_{1}+(\rho-\mu) \beta_{2} \beta_{1}\left(\beta_{1}-1\right) b_{2}^{\frac{1}{1-\alpha}}\right. \\
& \left.+\rho\left(\beta_{1}-1\right) \beta_{2}\left(\beta_{2}-1\right)-(\rho-\mu) \beta_{1} \beta_{2}\left(\beta_{2}-1\right) b_{2}^{\frac{1}{1-\alpha}}\right) \\
= & \frac{b_{2}^{\frac{\alpha-2}{1-\alpha}}\left(\beta_{2}-\alpha\right)\left(\beta_{1}-\alpha\right)\left(\beta_{2}-\beta_{1}\right)}{m(1-\alpha)}\left(\rho\left(\beta_{2}-1\right)\left(\beta_{1}-1\right)-(\rho-\mu) \beta_{2} \beta_{1} b_{2}^{\frac{1}{1-\alpha}}\right) \\
< & \frac{b_{2}^{\frac{\alpha-2}{1-\alpha}}\left(\beta_{2}-\alpha\right)\left(\beta_{1}-\alpha\right)\left(\beta_{2}-\beta_{1}\right)}{m(1-\alpha)}\left(\rho\left(\beta_{2}-1\right)\left(\beta_{1}-1\right)-(\rho-\mu) \beta_{2} \beta_{1}\right)=0 .
\end{aligned}
$$

Therefore, $b_{1}$ is on the upside of the single-peaked function, i.e., $\frac{\partial f\left(A_{1}, A_{2}, b_{1}\right)}{\partial b_{1}}>0$.

We show below that $\frac{d b_{1}}{d b_{2}}<0$. Because

$$
\frac{\partial f\left(A_{1}, A_{2}, b_{1}\right)}{\partial A_{1}} \frac{d A_{1}}{d b_{2}}+\frac{\partial f\left(A_{1}, A_{2}, b_{1}\right)}{\partial A_{2}} \frac{d A_{2}}{d b_{2}}+\frac{\partial f\left(A_{1}, A_{2}, b_{1}\right)}{\partial b_{1}} \frac{d b_{1}}{d b_{2}}=0,
$$


we have

$$
\frac{\partial f\left(A_{1}, A_{2}, b_{1}\right)}{\partial b_{1}} \frac{d b_{1}}{d b_{2}}=-\frac{\partial f\left(A_{1}, A_{2}, b_{1}\right)}{\partial A_{1}} \frac{d A_{1}}{d b_{2}}-\frac{\partial f\left(A_{1}, A_{2}, b_{1}\right)}{\partial A_{2}} \frac{d A_{2}}{d b_{2}}<0,
$$

where $\frac{\partial f\left(A_{1}, A_{2}, b_{1}\right)}{\partial A_{1}}>0, \frac{d A_{1}}{d b_{2}}>0, \frac{\partial f\left(A_{1}, A_{2}, b_{1}\right)}{\partial A_{2}}>0$, and $\frac{d A_{2}}{d b_{2}}>0$.

Step 3. We show that $b_{1}=b_{2}=1$ is a solution. If $b_{2}=1$, then it follows from step 1 that

$$
\left(\begin{array}{c}
A_{1} \\
A_{2}
\end{array}\right)=\left(\begin{array}{c}
\left(\beta_{2}-\alpha\right)\left(\mu \beta_{2}-\rho\right) \\
-\left(\beta_{1}-\alpha\right)\left(\mu \beta_{1}-\rho\right)
\end{array}\right) m^{-1}
$$

Substituting the above and $b_{1}=1$ into (A.17) yields

$$
\begin{aligned}
& \frac{1}{\rho \alpha}+A_{1}\left(1-\beta_{1}\right)+A_{2}\left(1-\beta_{2}\right) \\
= & \frac{1}{\rho \alpha}+A_{1}\left(1-\beta_{1}\right)+A_{2}\left(1-\beta_{2}\right) \\
= & \frac{1}{\rho \alpha}+\frac{\left(\beta_{2}-\alpha\right)\left(\mu \beta_{2}-\rho\right)\left(1-\beta_{1}\right)-\left(\beta_{1}-\alpha\right)\left(\mu \beta_{1}-\rho\right)\left(1-\beta_{2}\right)}{-\left(\beta_{1}-\alpha\right)\left(\beta_{2}-\alpha\right)\left(\beta_{2}-\beta_{1}\right) \rho(\rho-\mu)} \\
= & \frac{1}{\rho \alpha}+\frac{\mu\left(\beta_{2}+\beta_{1}\right)-(\alpha \mu+\rho)-\mu \beta_{1} \beta_{2}+\alpha \rho}{-\left(\beta_{1}-\alpha\right)\left(\beta_{2}-\alpha\right) \rho(\rho-\mu)} \\
= & \frac{1}{\rho \alpha}+\frac{\mu+\frac{\sigma^{2}(\alpha-1)}{2}}{\left(\rho-\alpha \mu-\frac{\sigma^{2} \alpha(\alpha-1)}{2}\right) \rho}=\kappa .
\end{aligned}
$$

Therefore, $b_{1}=b_{2}=1$ is a solution.

Step 4. We show that there is a unique solution $b_{2} \in(1, \bar{b})$. First, the function

$$
g\left(b_{2}\right) \equiv \frac{1}{\rho \alpha}\left(b_{1}\left(b_{2}\right)\right)^{\frac{\alpha}{1-\alpha}}+\left(1-\beta_{1}\right) A_{1}\left(b_{2}\right)\left(b_{1}\left(b_{2}\right)\right)^{\frac{\alpha-\beta_{1}}{1-\alpha}}+\left(1-\beta_{2}\right) A_{2}\left(b_{2}\right)\left(b_{1}\left(b_{2}\right)\right)^{\frac{\alpha-\beta_{2}}{1-\alpha}}
$$

is single-peaked in $b_{2}$, when variables $A_{1}, A_{2}, b_{1}$ are interpreted as functions of $b_{2}$. (Figure 8 plots the function $g\left(b_{2}\right)$.) We have

$$
g^{\prime}\left(b_{2}\right)=A_{1}^{\prime}\left(b_{2}\right)\left(1-\beta_{1}\right) b_{1}^{\frac{\alpha-\beta_{1}}{1-\alpha}}+A_{2}^{\prime}\left(b_{2}\right)\left(1-\beta_{2}\right) b_{1}^{\frac{\alpha-\beta_{2}}{1-\alpha}},
$$

where

$$
\begin{aligned}
& A_{1}^{\prime}\left(b_{2}\right)=b_{2}^{\frac{\beta_{1}-1}{1-\alpha}-1} \frac{\beta_{2}-\alpha}{1-\alpha}\left(\rho\left(\beta_{2}-1\right)\left(\beta_{1}-1\right)-(\rho-\mu) \beta_{2} \beta_{1} b_{2}^{\frac{1}{1-\alpha}}\right) m^{-1}, \\
& A_{2}^{\prime}\left(b_{2}\right)=-b_{2}^{\frac{\beta_{2}-1}{1-\alpha}}-1 \frac{\beta_{1}-\alpha}{1-\alpha}\left(\rho\left(\beta_{2}-1\right)\left(\beta_{1}-1\right)-(\rho-\mu) \beta_{2} \beta_{1} b_{2}^{\frac{1}{1-\alpha}}\right) m^{-1} .
\end{aligned}
$$

Hence the sign of $g^{\prime}\left(b_{2}\right)$ equals that of

$$
\left(\beta_{2}-\alpha\right)\left(1-\beta_{1}\right) b^{\frac{\beta_{2}-\beta_{1}}{1-\alpha}}+\left(\alpha-\beta_{1}\right)\left(1-\beta_{2}\right) b_{2}^{\frac{\beta_{2}-\beta_{1}}{1-\alpha}}
$$


which decrease in $b_{2}$ because $b_{1}^{\frac{\beta_{2}-\beta_{1}}{1-\alpha}}$ decreases in $b_{2}, b_{2}^{\beta_{2}-\beta_{1}}$ increases in $b_{2}$, and $\left(\alpha-\beta_{1}\right)\left(1-\beta_{2}\right)<$ 0 . Second, $g\left(b_{2}\right)>\kappa$ when $b_{2}-1>0$ is small. Because $g(1)=\kappa$, it is sufficient to show that $g^{\prime}\left(b_{2}=1\right)>0$. The sign of $g^{\prime}\left(b_{2}\right)$ is positive because setting $b_{1}=b_{2}=1$ in (A.21) yields

$$
\left(\beta_{2}-\alpha\right)\left(1-\beta_{1}\right)+\left(\alpha-\beta_{1}\right)\left(1-\beta_{2}\right)=(1-\alpha)\left(\beta_{2}-\beta_{1}\right)>0
$$

Third, we show that

$$
\lim _{b_{2} \uparrow \bar{b}} g\left(b_{2}\right)<\kappa .
$$

It follows from the formula for $A_{2}$ that $\lim _{b_{2} \uparrow \bar{b}} A_{2}=0$. It follows from $b_{1}^{\frac{\beta_{2}}{1-\alpha}} \leq \rho\left(-A_{2}\right)(1-$ $\left.\beta_{2}\right)\left(\alpha-\beta_{2}\right)$ that $\lim _{b_{2} \uparrow \bar{b}} b_{1}=0$. Thus,

$$
\lim _{b_{2} \uparrow \bar{b}} g\left(b_{2}\right)=\lim _{b_{2} \uparrow \bar{b}} \frac{1}{\rho \alpha} b_{1}^{\frac{\alpha}{1-\alpha}}+A_{2}\left(1-\beta_{2}\right) b_{1}^{\frac{\alpha-\beta_{2}}{1-\alpha}}=\lim _{b_{2} \uparrow \bar{b}}\left[\frac{1}{\rho \alpha}+\frac{1}{\rho\left(\beta_{2}-\alpha\right)}\right] b_{1}^{\frac{\alpha}{1-\alpha}} .
$$

If $\alpha>0$, then the above limit is zero and $\kappa>0$. If $\alpha<0$, then $\left[\frac{1}{\rho \alpha}+\frac{1}{\rho\left(\beta_{2}-\alpha\right)}\right]<0$ and the above limit is $-\infty$. In both cases, the limit is less than $\kappa$. The Intermediate Value Theorem implies the existence of $b_{2} \in(1, \bar{b})$ such that $g\left(b_{2}\right)=\kappa$. This solution is unique because $g\left(b_{2}\right)$ is single-peaked and $g(1)=\kappa$.

Step 5. If there is a solution such that $b_{2}>\bar{b}$, then according to step $1, A_{2}>0$. The function $f$ in step 2 would be monotonically decreasing because

$$
\frac{\partial f}{\partial b}=\frac{b^{\frac{-\beta_{2}}{1-\alpha}-1}}{1-\alpha}\left(-A_{1} \beta_{1}\left(1-\beta_{1}\right)\left(\alpha-\beta_{1}\right) b^{\frac{\beta_{2}-\beta_{1}}{1-\alpha}}-A_{2} \beta_{2}\left(1-\beta_{2}\right)\left(\alpha-\beta_{2}\right)\right)<0 .
$$

This implies that $f\left(A_{1}, A_{2}, b\right)=0$ has a unique solution and hence $b_{1}=b_{2}>1$. If $b_{1}=b_{2}$, then $g\left(b_{2}\right)$ in step 4 increases in $b_{2}$. This is because $g^{\prime}\left(b_{2}\right)$ has the same sign as

$$
\left(\beta_{2}-\alpha\right)\left(1-\beta_{1}\right) b_{2}^{\frac{\beta_{2}-\beta_{1}}{1-\alpha}}+\left(\alpha-\beta_{1}\right)\left(1-\beta_{2}\right) b_{2}^{\frac{\beta_{2}-\beta_{1}}{1-\alpha}}=b_{2}^{\frac{\beta_{2}-\beta_{1}}{1-\alpha}}\left(\beta_{2}-\beta_{1}\right)(1-\alpha)>0 .
$$

Therefore, $g\left(b_{2}\right)>g(1)=\kappa$, which means that $b_{1}=b_{2}>1$ violates (A.17). Q.E.D.

Proof of Proposition 3: (necessity) Suppose that a non-autarkic risk sharing contract exists. First, we observe that $\bar{U}^{a}(y)=\bar{U}^{a}(1)$ and $U^{p}(y, v)=y U^{p}(1, v)$ for all $y>0$. This is because the income process is homogeneous of degree one in its initial condition. More specifically, consider two income processes with initial conditions 1 and $y$, respectively. Denote the former as $\left(\left\{y_{t}\right\}_{t \geq 0}, y_{0}=1\right)$ and the latter as $\left\{y y_{t}\right\}_{t \geq 0}$. If $\left\{c_{t}\right\}_{t \geq 0}$ is a contract under $\left(\left\{y_{t}\right\}_{t \geq 0}, y_{0}=1\right)$, then $\left\{y c_{t}\right\}_{t \geq 0}$ is a contract under income $\left\{y y_{t}\right\}_{t \geq 0}$ such that the agent's surplus remains unchanged because $\ln \left(y c_{t}\right)-\ln \left(y y_{t}\right)=\ln \left(c_{t}\right)-\ln \left(y_{t}\right)$, and the principal's profit is $y$ times his profit under the contract $\left\{c_{t}\right\}_{t \geq 0}$ because $y y_{t}-y c_{t}=y\left(y_{t}-c_{t}\right)$. 
Next, setting $y=1$ in the Bellman equation presented in Section 3.3.5 and using the above homogeneity property, we obtain

$$
\begin{array}{ll}
U^{p}(1, v)=\max _{v_{b}, v_{g}} & \left(1-e^{\left(v-\frac{1}{1+\rho d t}\left(0.5 v_{b}+0.5 v_{g}\right)\right) / d t}\right) d t \\
& +\frac{1}{1+\rho d t}\left[0.5(1-\sigma \sqrt{d t}) U^{p}\left(1, v_{b}\right)+0.5(1+\sigma \sqrt{d t}) U^{p}\left(1, v_{g}\right)\right] \\
\text { subject to } & v_{b} \in\left[0, \bar{U}^{a}(1)\right], \quad v_{g} \in\left[0, \bar{U}^{a}(1)\right] .
\end{array}
$$

By the fact that $1-x \leq e^{-x}$ for any $x$,

$$
\left(1-e^{\left(v-\frac{1}{1+\rho d t}\left(0.5 v_{b}+0.5 v_{g}\right)\right) / d t}\right) d t \leq \frac{1}{1+\rho d t}\left(0.5 v_{b}+0.5 v_{g}\right)-v
$$

with equality if and only if $\frac{1}{1+\rho d t}\left(0.5 v_{b}+0.5 v_{g}\right)=v$. Thus, $U^{p}(1, v)$ is below the value function $M(v)$ defined in the following problem:

$$
\begin{aligned}
M(v)=\max _{v_{b}, v_{g}} & \frac{1}{1+\rho d t}\left(0.5 v_{b}+0.5 v_{g}\right)-v \\
& +\frac{1}{1+\rho d t}\left[0.5(1-\sigma \sqrt{d t}) M\left(v_{b}\right)+0.5(1+\sigma \sqrt{d t}) M\left(v_{g}\right)\right] \\
\text { subject to } & v_{b} \in\left[0, \bar{U}^{a}(1)\right], \quad v_{g} \in\left[0, \bar{U}^{a}(1)\right] .
\end{aligned}
$$

Solving the above linear Bellman equation yields

$$
M(v)=M(0)-v=\frac{0.5 \sigma \sqrt{d t}}{\rho d t} \bar{U}^{a}(1)-v .
$$

In addition, the solution satisfies $\frac{1}{1+\rho d t}\left(0.5 v_{b}+0.5 v_{g}\right) \neq v$. Thus, $U^{p}(1, v)<M(v)$.

Because $U^{p}\left(1, \bar{U}^{a}(1)\right)=0$ and $U^{p}\left(1, \bar{U}^{a}(1)\right)<M\left(\bar{U}^{a}(1)\right)$, we have

$$
0<M\left(\bar{U}^{a}(1)\right)=\frac{0.5 \sigma \sqrt{d t}-\rho d t}{r \sqrt{d t}} \bar{U}^{a}(1) .
$$

When a non-autarkic risk sharing contract exists, $\bar{U}^{a}(1)>0$. The above inequality implies $\sigma \sqrt{d t}>2 \rho d t$.

(sufficiency) Suppose $\sigma \sqrt{d t}>2 \rho d t$. Consider a contract (not necessarily optimal) in which the agent's consumption satisfies

$$
\ln \left(c_{t}\right)-\ln \left(y_{t}\right)= \begin{cases}-\epsilon, & \text { if } \frac{y_{t}}{y_{t-d t}}=1+\sigma \sqrt{d t} \\ (1+2 \rho d t) \epsilon, & \text { if } \frac{y_{t}}{y_{t-d t}}=1-\sigma \sqrt{d t}\end{cases}
$$

Under a good shock (i.e., $\frac{y_{t}}{y_{t-d t}}=1+\sigma \sqrt{d t}$ ), denote the principal and the agent's continuation values as $U_{g}^{p}\left(y_{t}, \epsilon\right)$ and $U_{g}^{a}\left(y_{t}, \epsilon\right)$. Similarly, denote the continuation values under a bad shock 
as $U_{b}^{p}\left(y_{t}, \epsilon\right)$ and $U_{b}^{a}\left(y_{t}, \epsilon\right)$. They satisfy the Bellman equations:

$$
\begin{aligned}
U_{g}^{p}(y, \epsilon) & =\left(1-e^{-\epsilon}\right) y d t+\frac{1}{1+\rho d t}\left[\frac{1}{2} U_{g}^{p}((1+\sigma \sqrt{d t}) y, \epsilon)+\frac{1}{2} U_{b}^{p}((1-\sigma \sqrt{d t}) y, \epsilon)\right], \\
U_{b}^{p}(y, \epsilon) & =\left(1-e^{(1+2 \rho d t) \epsilon}\right) y d t+\frac{1}{1+\rho d t}\left[\frac{1}{2} U_{g}^{p}((1+\sigma \sqrt{d t}) y, \epsilon)+\frac{1}{2} U_{b}^{p}((1-\sigma \sqrt{d t}) y, \epsilon)\right], \\
U_{g}^{a}(y, \epsilon) & =(\ln (y)-\epsilon) d t+\frac{1}{1+\rho d t}\left[\frac{1}{2} U_{g}^{a}((1+\sigma \sqrt{d t}) y)+\frac{1}{2} U_{b}^{a}((1-\sigma \sqrt{d t}) y)\right], \\
U_{b}^{a}(y, \epsilon) & =(\ln (y)+(1+2 \rho d t) \epsilon) d t+\frac{1}{1+\rho d t}\left[\frac{1}{2} U_{g}^{a}((1+\sigma \sqrt{d t}) y)+\frac{1}{2} U_{g}^{a}((1-\sigma \sqrt{d t}) y)\right] .
\end{aligned}
$$

We can verify that $U_{g}^{a}(y, \epsilon)=U_{d}(y)$ and $U_{b}^{a}(y, \epsilon)=U_{d}(y)+2(1+\rho d t) \epsilon d t$, hence the agent's participation constraints are satisfied. Next we examine the principal's participation constraints $U_{g}^{p}(y, \epsilon) \geq 0$ and $U_{b}^{p}(y, \epsilon) \geq 0$. Since $U_{g}^{p}(y, \epsilon)>U_{b}^{p}(y, \epsilon)$, it is sufficient to check whether $U_{b}^{p}(y, \epsilon) \geq 0$ only.

We can easily guess and verify that $U_{g}^{p}(y, \epsilon)=U_{g}^{p}(1, \epsilon) y$ and $U_{b}^{p}(y, \epsilon)=U_{b}^{p}(1, \epsilon) y$ for all $y>0$. Hence, the above Bellman equations can be rewritten as

$$
\begin{aligned}
U_{g}^{p}(y, \epsilon) & =\left(1-e^{-\epsilon}\right) y d t+\frac{1}{1+\rho d t}\left(\frac{1}{2}(1+\sigma \sqrt{d t}) U_{g}^{p}(y, \epsilon)+\frac{1}{2}(1-\sigma \sqrt{d t}) U_{b}^{p}(y, \epsilon)\right), \\
U_{b}^{p}(y, \epsilon) & =\left(1-e^{(1+2 \rho d t) \epsilon}\right) y d t+\frac{1}{1+\rho d t}\left(\frac{1}{2}(1+\sigma \sqrt{d t}) U_{g}^{p}(y, \epsilon)+\frac{1}{2}(1-\sigma \sqrt{d t}) U_{b}^{p}(y, \epsilon)\right) .
\end{aligned}
$$

Solving $U_{b}^{p}(y, \epsilon)$ yields

$$
U_{b}^{p}(y, \epsilon)=\left((1+\rho d t)\left(1-e^{(1+2 \rho d t) \epsilon}\right)+\frac{1+\sigma \sqrt{d t}}{2}\left(e^{(1+2 \rho d t) \epsilon}-e^{-\epsilon}\right)\right) \frac{y}{\rho} .
$$

When $\epsilon=0, U_{b}^{p}(y, \epsilon)=0$. We can then compute that

$$
\begin{aligned}
\left.\frac{\partial U_{b}^{p}(y, \epsilon)}{\partial \epsilon}\right|_{\epsilon=0} & =\left(-(1+\rho d t)(1+2 \rho d t)+\frac{1+\sigma \sqrt{d t}}{2}(2+2 \rho d t)\right) \frac{y}{\rho} \\
& =\frac{y(1+\rho d t)}{r}(\sigma \sqrt{d t}-2 \rho d t)>0 .
\end{aligned}
$$

This shows that the non-autarkic risk-sharing contract constructed before is enforceable and the principal is better off. Q.E.D.

\section{B Additional Proofs for Section 2.5}

In this appendix, we verify that the solution in Section 2.5 satisfies the conditions in Theorem 3. This consists of six steps. 
Step 1. We verify the transversality condition $(29)$. For $y \leq(z / b)^{\frac{1}{1-\alpha}}$,

$$
\begin{aligned}
|J(y, z)| & =\left|\frac{y}{r-\mu}+\frac{(1-\alpha)^{2}}{(\rho-\alpha r) \alpha} z^{\frac{1}{1-\alpha}}+A z^{\frac{1-\beta}{1-\alpha}} y^{\beta}\right| \\
& \leq\left(\frac{1}{(r-\mu) b^{\frac{1}{1-\alpha}}}+\frac{(1-\alpha)^{2}}{(\rho-\alpha r)|\alpha|}+\frac{|A|}{b^{\frac{\beta}{1-\alpha}}}\right) z^{\frac{1}{1-\alpha}} .
\end{aligned}
$$

The integrability condition (16) implies that

$$
E\left[\int_{0}^{\infty} e^{-r t}\left|\frac{1-\alpha}{\alpha} Z_{t}^{\frac{1}{1-\alpha}}\right| d t\right]<\infty .
$$

We then deduce that

$$
\begin{aligned}
0 & =\lim _{t \rightarrow \infty} E\left[\int_{t}^{\infty} e^{-r s} Z_{s}^{\frac{1}{1-\alpha}} d s\right]=\lim _{t \rightarrow \infty} E\left[\int_{t}^{\infty} e^{-r s}\left(e^{(r-\rho) s} X_{s}\right)^{\frac{1}{1-\alpha}} d s\right] \\
& \geq \lim _{t \rightarrow \infty} E\left[X_{t}^{\frac{1}{1-\alpha}} \int_{t}^{\infty} e^{-r s+\frac{r-\rho}{1-\alpha} s} d s\right]=\frac{\rho-\alpha r}{1-\alpha} \lim _{t \rightarrow \infty} E\left[e^{-r t} Z_{t}^{\frac{1}{1-\alpha}}\right]
\end{aligned}
$$

where the inequality follows from the fact that $X$ is a nonnegative increasing process and where we have used the fact that $\rho>\alpha r$. Thus,

$$
\lim _{t \rightarrow \infty} E\left[e^{-r t} Z_{t}^{\frac{1}{1-\alpha}}\right]=0
$$

implying that the transversality condition $\lim _{t \rightarrow \infty} E\left[e^{-r t} J\left(Y_{t}, Z_{t}\right)\right]=0$ holds for $Y_{t} \leq\left(Z_{t} / b\right)^{\frac{1}{1-\alpha}}$. For $z<b y^{1-\alpha}$, equation (41) implies that

$$
|J(y, z)|<K y
$$

for some constant $K>0$. Thus, the transversality condition holds for $Y_{t}>\left(Z_{t} / b\right)^{\frac{1}{1-\alpha}}$ by Assumption 1.

Step 2. We check condition (28). It is sufficient to show that

$$
E\left[\int_{0}^{\infty}\left(e^{-r t} J_{y}\left(Y_{t}, Z_{t}\right) \sigma Y_{t}\right)^{2} d t\right]<\infty .
$$

We can show that

$$
\left|J_{y}(y, z)\right|=\left|\frac{1}{r-\mu}+A z^{\frac{1-\beta}{1-\alpha}} y^{\beta-1} \beta\right| \leq\left|\frac{1}{r-\mu}\right|+|A| b^{\frac{1-\beta}{1-\alpha}}, \quad \text { for } z \geq b y^{1-\alpha},
$$

and

$$
\left|J_{y}(y, z)\right|=\left|z U_{d}^{\prime}(y)+K_{1}\right| \leq K_{2}, \quad \text { for } z<b y^{1-\alpha},
$$

where $K_{1}$ and $K_{2}$ are some constant terms. Thus, we only need to verify

$$
E\left[\int_{0}^{\infty}\left(e^{-r t} Y_{t}\right)^{2} d t\right]<\infty
$$


This is true if $r>\mu+\sigma^{2} / 2$.

Step 3. We verify that the dual value function $J$ in (41) satisfies the variational inequality in the jump region. That is, if $z<b y^{1-\alpha}$, then

$$
r J(y, z)<y+\frac{1-\alpha}{\alpha} z^{\frac{1}{1-\alpha}}+(r-\rho) z J_{z}(y, z)+J_{y}(y, z) \mu y+\frac{\sigma^{2}}{2} y^{2} J_{y y}(y, z) .
$$

Because the left-hand side equals the right-hand side at $z=b y^{1-\alpha}$, it is sufficient to show that the derivative of the left-hand side with respect to $z$ is above that of the right-hand side with respect to $z$. That is,

$$
r J_{z}(y, z)>\frac{z^{\frac{\alpha}{1-\alpha}}}{\alpha}+(r-\rho) J_{z}(y, z)+(r-\rho) z J_{z z}(y, z)+J_{y z}(y, z) \mu y+\frac{\sigma^{2}}{2} y^{2} J_{y y z}(y, z) .
$$

It follows from $J_{z}(y, z)=U_{d}(y)$ that $J_{z z}(y, z)=0, J_{y z}(y, z)=U_{d}^{\prime}(y)$, and $J_{y y z}(y, z)=U_{d}^{\prime \prime}(y)$. The above inequality becomes

$$
r U_{d}(y)>\frac{z^{\frac{\alpha}{1-\alpha}}}{\alpha}+(r-\rho) U_{d}(y)+U_{d}^{\prime}(y) \mu y+\frac{\sigma^{2}}{2} y^{2} U_{d}^{\prime \prime}(y)
$$

which, after simplification, is

$$
\frac{y^{\alpha}}{\alpha}>\frac{z^{\frac{\alpha}{1-\alpha}}}{\alpha} .
$$

To prove this inequality, we will show $b<1$, which implies that $y^{1-\alpha}>b y^{1-\alpha}>z$ as desired. By (35) and (40), to prove that

$$
b=\left(\frac{(\rho-\alpha r)(\beta-\alpha)}{\beta(1-\alpha)\left(\rho-\alpha \mu-\alpha(\alpha-1) \sigma^{2} / 2\right)}\right)^{\frac{1-\alpha}{\alpha}}<1,
$$

it is sufficient to show that $\frac{(\rho-\alpha r)(\beta-\alpha)}{\beta(1-\alpha)\left(\rho-\alpha \mu-\alpha(\alpha-1) \sigma^{2} / 2\right)}$ is less than 1 when $\alpha>0$, and is greater than 1 when $\alpha<0$. Because both the numerator and the denominator are positive, it is equivalent to proving that $\beta(1-\alpha)\left(\rho-\alpha \mu-\alpha(\alpha-1) \sigma^{2} / 2\right)-(\rho-\alpha r)(\beta-\alpha)$ has the same sign as $\alpha$. We can show that

$$
\begin{aligned}
& \beta(1-\alpha)\left(\rho-\alpha \mu-\alpha(\alpha-1) \sigma^{2} / 2\right)-(\rho-\alpha r)(\beta-\alpha) \\
= & \beta \alpha(1-\alpha)\left(-\mu+(1-\alpha) \sigma^{2} / 2\right)+\beta(1-\alpha) \rho-(\rho-\alpha r)(\beta-\alpha) \\
= & \beta \alpha(1-\alpha)\left(-\mu+(1-\alpha) \sigma^{2} / 2\right)+\alpha((1-\beta)(\rho-r)+(1-\alpha) r) \\
= & \beta \alpha(1-\alpha)\left(-\mu+(1-\alpha) \sigma^{2} / 2+\frac{(1-\beta)(\rho-r)}{(1-\alpha) \beta}+\frac{r}{\beta}\right) \\
= & \beta \alpha(1-\alpha)\left(-\mu+(1-\alpha) \sigma^{2} / 2+\mu+\sigma^{2}(\beta-1) / 2\right) \\
= & \beta \alpha(1-\alpha) \sigma^{2}(\beta-\alpha) / 2,
\end{aligned}
$$


where the fourth equality uses (38). The sign of the last line is determined by $\alpha$ because $\beta(1-\alpha) \sigma^{2}(\beta-\alpha)>0$.

Step 4. We verify that $J_{z}(y, z) \geq U_{d}(y)$ for all $(z, y)$ in the no-jump region for $J$ defined in (37). If $z \geq b y^{1-\alpha}$, then $J_{z}(y, z) \geq J_{z}\left(y, b y^{1-\alpha}\right)=U_{d}(y)$, where the inequality follows from $J_{z z} \geq 0$ and the equality follows from the value-matching condition.

Step 5. By the solution in Section 2.5, (30) and (31) hold. We need to check that $X^{*} \in \mathcal{I}(z)$ and the integrability conditions stated in the theorem hold. Since $\tilde{u}(z)=\frac{1-\alpha}{\alpha} z^{\frac{1}{1-\alpha}}$, we need to show

$$
\begin{aligned}
& E\left[\int_{0}^{\infty} e^{-r t}\left(Z_{t}^{*}\right)^{\frac{1}{1-\alpha}} d t\right]<\infty, \\
& E\left[\int_{0}^{\infty} e^{-\rho t}\left|U_{d}\left(Y_{t}\right)\right| d X_{t}^{*}\right]<\infty, \\
& E\left[\int_{0}^{\infty} e^{-r t}\left(e^{(r-\rho) t}\left(X_{t}^{*}+\delta\right)\right)^{\frac{1}{1-\alpha}} d t\right]<\infty, \\
& E\left[\int_{0}^{\infty} e^{-r t}\left(e^{(r-\rho) t}\left(X_{t}^{*}(1+\delta)\right)\right)^{\frac{1}{1-\alpha}} d t\right]<\infty, \\
& E\left[\int_{0}^{\infty} e^{-r t}\left(e^{(r-\rho) t}\left(X_{t}^{*}(1-\delta)\right)\right)^{\frac{1}{1-\alpha}} d t\right]
\end{aligned}
$$

It is sufficient to check (B.1) and (B.2) since the last two integrals can be similarly checked using $Z_{t}^{*}=e^{(r-\rho) t} X_{t}^{*}$ and since we can derive

$$
\begin{aligned}
E\left[\int_{0}^{\infty} e^{-r t}\left(e^{(r-\rho) t}\left(X_{t}^{*}+\delta\right)\right)^{\frac{1}{1-\alpha}} d t\right] & <E\left[\int_{0}^{\infty} e^{-r t}\left(e^{(r-\rho) t}\left(X_{t}^{*}\left(1+\delta / X_{0}^{*}\right)\right)\right)^{\frac{1}{1-\alpha}} d t\right] \\
& =(1+\delta / z)^{\frac{1}{1-\alpha}} E\left[\int_{0}^{\infty} e^{-r t}\left(Z_{t}^{*}\right)^{\frac{1}{1-\alpha}} d t\right] .
\end{aligned}
$$

To check (B.1), it suffices to use (43) to show

$$
E\left[\int_{0}^{\infty} e^{-\frac{\rho-\alpha r}{1-\alpha} t} M_{t} d t\right]<\infty, \quad \text { where } M_{t}=\sup _{s \in[0, t]} Y_{s} e^{\frac{(\rho-r) s}{1-\alpha}} .
$$

We will show that

$$
E\left[\int_{0}^{\infty} e^{-\frac{\rho-\alpha r}{1-\alpha} t} M_{t} d t\right]=\frac{E\left[\int_{0}^{\infty} M_{t} d\left(-e^{-\frac{\rho-\alpha r}{1-\alpha} t}\right)\right]}{\frac{\rho-\alpha r}{1-\alpha}}<\infty .
$$

Pick some $\epsilon>0$, and for $n=0,1,2, \ldots$ define a sequence of stopping times $\tau_{n} \equiv \inf _{t \geq 0}\left\{t: M_{t}=\right.$ $\left.Y_{0}(1+\epsilon)^{n}\right\}$. Since $M_{t} \leq Y_{0}(1+\epsilon)^{n+1}$ for $t \in\left[\tau_{n}, \tau_{n+1}\right)$,

$$
\begin{aligned}
E\left[\int_{0}^{\infty} M_{t} d\left(-e^{-\frac{\rho-\alpha r}{1-\alpha} t}\right)\right] & \leq E\left[\sum_{n=0}^{\infty} Y_{0}(1+\epsilon)^{n+1}\left(e^{-\frac{\rho-\alpha r}{1-\alpha} \tau_{n}}-e^{-\frac{\rho-\alpha r}{1-\alpha} \tau_{n+1}}\right)\right] \\
& =\sum_{n=0}^{\infty} Y_{0}(1+\epsilon)^{n+1}\left(E\left[e^{-\frac{\rho-\alpha r}{1-\alpha} \tau_{n}}\right]-E\left[e^{-\frac{\rho-\alpha r}{1-\alpha} \tau_{n+1}}\right]\right) .
\end{aligned}
$$


By Harrison (1985) or Stokey (2008), we can compute

$$
E\left[e^{-\frac{\rho-\alpha r}{1-\alpha} \tau_{n}}\right]=(1+\epsilon)^{-\beta n},
$$

where $\beta>1$ satisfies (38). Therefore,

$$
\begin{aligned}
& \sum_{n=0}^{\infty} Y_{0}(1+\epsilon)^{n+1}\left(E\left[e^{-\frac{\rho-\alpha r}{1-\alpha} \tau_{n}}\right]-E\left[e^{-\frac{\rho-\alpha r}{1-\alpha} \tau_{n+1}}\right]\right) \\
= & Y_{0}\left((1+\epsilon)^{\beta}-1\right) \sum_{n=0}^{\infty}\left((1+\epsilon)^{1-\beta}\right)^{n+1}<\infty,
\end{aligned}
$$

as desired.

To show (B.2), we use $U_{d}(y)=\kappa y^{\alpha}$ and define

$$
G(y, z) \equiv E\left[\int_{0}^{\infty} e^{-\rho t} Y_{t}^{\alpha} d X_{t}^{*}\right], \quad \text { for } z \geq b y^{1-\alpha} .
$$

Then, as in the proof of Theorem 3 for the HJB equation, $G$ satisfies

$$
r G(y, z)=G_{z}(y, z)(r-\rho) z+G_{y}(y, z) \mu y+\frac{\sigma^{2}}{2} G_{y y}(y, z) y^{2},
$$

subject to

$$
\left.G_{z}(y, z)\right|_{z=b y^{1-\alpha}}=-y^{\alpha} .
$$

Solving yields:

$$
G(y, z)=b^{\frac{\beta-\alpha}{1-\alpha}} \frac{1-\alpha}{\beta-1} z^{\frac{1-\beta}{1-\alpha}} y^{\beta} .
$$

We also need to check that this solution satisfies the transversality condition:

$$
\lim _{t \rightarrow \infty} E\left[e^{-r t} G\left(Y_{t}, Z_{t}^{*}\right)\right]=0
$$

We only need to show that

$$
\lim _{t \rightarrow \infty} E\left[e^{-r t}\left(Z_{t}^{*}\right)^{\frac{1-\beta}{1-\alpha}} Y_{t}^{\beta}\right]=0 .
$$

This follows from

$$
\lim _{t \rightarrow \infty} E\left[e^{-r t}\left(Z_{t}^{*}\right)^{\frac{1-\beta}{1-\alpha}} Y_{t}^{\beta}\right] \leq \lim _{t \rightarrow \infty} E\left[e^{-r t}\left(b Y_{t}^{1-\alpha}\right)^{\frac{1-\beta}{1-\alpha}} Y_{t}^{\beta}\right]=\lim _{t \rightarrow \infty} E\left[b^{\frac{1-\beta}{1-\alpha}} e^{-r t} Y_{t}\right]=0
$$

where the last equality follows from $r>\mu$ and where we have used the fact that $\beta>1$ and $Z_{t}^{*} \geq b Y_{t}^{1-\alpha}$.

Step 6. We show that $J(y, z)$ is strictly convex in $z$ in the no-jump region. We also derive a unique solution to $(42)$. 
The convexity follows from the fact that

$$
\begin{aligned}
J_{z z}(y, z) & =\frac{1}{(\rho-r \alpha)} z^{\frac{2 \alpha-1}{1-\alpha}}+A \frac{1-\beta}{1-\alpha} \frac{\alpha-\beta}{1-\alpha} z^{\frac{2 \alpha-\beta-1}{1-\alpha}} y^{\beta} \\
& >\frac{1}{(\rho-r \alpha)} z^{\frac{2 \alpha-1}{1-\alpha}}+A \frac{1-\beta}{1-\alpha} \frac{\alpha-\beta}{1-\alpha} z^{\frac{2 \alpha-\beta-1}{1-\alpha}}\left(\frac{z}{b}\right)^{\frac{\beta}{1-\alpha}}=0,
\end{aligned}
$$

for $z>b y^{1-\alpha}$, where the last equality uses the super-contact condition. Because $J_{z}(y, z)$ is strictly increasing in $z$ and

$$
\lim _{z \rightarrow \infty} J_{z}(y, z)= \begin{cases}\infty & \text { if } \alpha>0 \\ 0 & \text { if } \alpha<0\end{cases}
$$

it follows from the Intermediate Value Theorem that the solution for $z$ exists in equation (42) as long as $w$ belongs to the range of the utility function (i.e., $w \in\left[U_{d}(y), \infty\right)$ when $\alpha>0$ and $w \in\left[U_{d}(y), 0\right)$ when $\left.\alpha<0\right)$.

\section{Example III}

In this appendix, we solve an example from Ljungqvist and Sargent (2004, Chapter 20) in which both the principal and the agent are risk averse. We consider a symmetric setup. Let $u^{p}(c)=u(c)=-e^{-\gamma c}$, where $\gamma>0$ represents the coefficient of absolute risk aversion. The agent and the principal have incomes $Y_{t}=\sigma B_{t}$ and $-Y_{t}=-\sigma B_{t}$, respectively, where $B_{t}$ is a standard Brownian motion. In this case, their incomes are perfectly negatively correlated. This example does not satisfy some assumptions in our general theory developed before. In particular, consumption can be negative. Nevertheless, our key insights still apply and we shall proceed to derive the efficient contract since exponential utility is widely used in the contracting literature.

For this example, the dual function is given by

$$
\tilde{u}(x, h)=\max _{c}(1+h) u(-c)+x u(c)=-2 \sqrt{x(1+h)},
$$

and the optimal consumption rule is given by

$$
c^{*}=\frac{1}{2 \gamma} \ln \left(\frac{x}{1+h}\right) \text {. }
$$

Let the outside value be the autarky value so that

$$
U_{d}(y)=\kappa e^{-\gamma y}, \quad U_{d}^{p}(-y)=\kappa e^{\gamma y}, \quad y \in \mathbb{R},
$$

where $\kappa \equiv-\left(\rho-\gamma^{2} \sigma^{2} / 2\right)^{-1}<0$. We assume $\rho>\gamma^{2} \sigma^{2} / 2$ so that the autarky value is finite. 
Conjecture that the no-jump region is given by

$$
\left\{(y, x, h) \in \mathbb{R} \times \mathbb{R}_{++} \times \mathbb{R}_{+}: \frac{x}{1+h} \in\left[b e^{2 \gamma y}, b^{-1} e^{2 \gamma y}\right]\right\}
$$

where $0<b<1$ is a constant to be determined. We can verify that the dual value function in the no-jump region takes the following form:

$$
\tilde{V}(y, x, h)=\frac{-2 \sqrt{x(1+h)}}{\rho}+A x^{\frac{1-\beta}{2}}(1+h)^{\frac{1+\beta}{2}} e^{\gamma \beta y}+A(1+h)^{\frac{1-\beta}{2}} x^{\frac{1+\beta}{2}} e^{-\gamma \beta y},
$$

where $\beta \equiv \sqrt{2 \rho}(\gamma \sigma)^{-1}>1$ and $A$ is a constant to be determined.

In the jump region, we can verify that for $x<b e^{2 \gamma y}(1+h)$,

$$
\tilde{V}(y, x, h)=\left(x-(1+h) b e^{2 \gamma y}\right) U_{d}(y)+\tilde{V}\left(y,(1+h) b e^{2 \gamma y}, h\right),
$$

and for $x>b^{-1} e^{2 \gamma y}(1+h)$,

$$
\tilde{V}(y, x, h)=\left(1+h-x b e^{-2 \gamma y}\right) U_{d}^{p}(-y)+\tilde{V}\left(y, x, x b e^{-2 \gamma y}-1\right) .
$$

The constants $A$ and $b$ are determined by the value-matching and super-contact conditions. Due to symmetry, we only need to use these conditions on one of the two boundaries. Without loss of generality, we use the lower boundary. By the value-matching condition, $\lim _{\frac{x}{1+h} \downarrow b e^{2 \gamma y}} \tilde{V}_{x}(y, x, h)=U_{d}(y)$, and the super-contact condition, $\lim _{\frac{x}{1+h} \downarrow b e^{2 \gamma y}} \tilde{V}_{x x}(y, x, h)=0$, we can derive

$$
\begin{aligned}
-\frac{2}{\rho}+A\left((1-\beta) b^{\frac{-\beta}{2}}+(1+\beta) b^{\frac{\beta}{2}}\right) & =\kappa, \\
\frac{2}{\rho}+A\left(\beta^{2}-1\right)\left(b^{\frac{\beta}{2}}+b^{-\frac{\beta}{2}}\right) & =0 .
\end{aligned}
$$

Simplifying yields one equation for $b$,

$$
\frac{2 \beta}{\beta^{2}-1} \frac{1}{1+b^{\beta}}-\rho \kappa b^{\frac{1}{2}}-\frac{\beta}{\beta-1}=0 .
$$

Proposition 4 Suppose that $\gamma>0, \sigma>0$, and $\rho>\gamma^{2} \sigma^{2} / 2$. Then there are two solutions to the above equation. One satisfies $b \in(0,1)$ and the other is degenerate $(b=1)$.

Proof: $\quad$ Set $l=b^{\frac{1}{2}}$ and rewrite (C.1) as

$$
\frac{2 \beta}{\beta^{2}-1} \frac{1}{1+l^{2 \beta}}-\rho \kappa l-\frac{\beta}{\beta-1}=0
$$




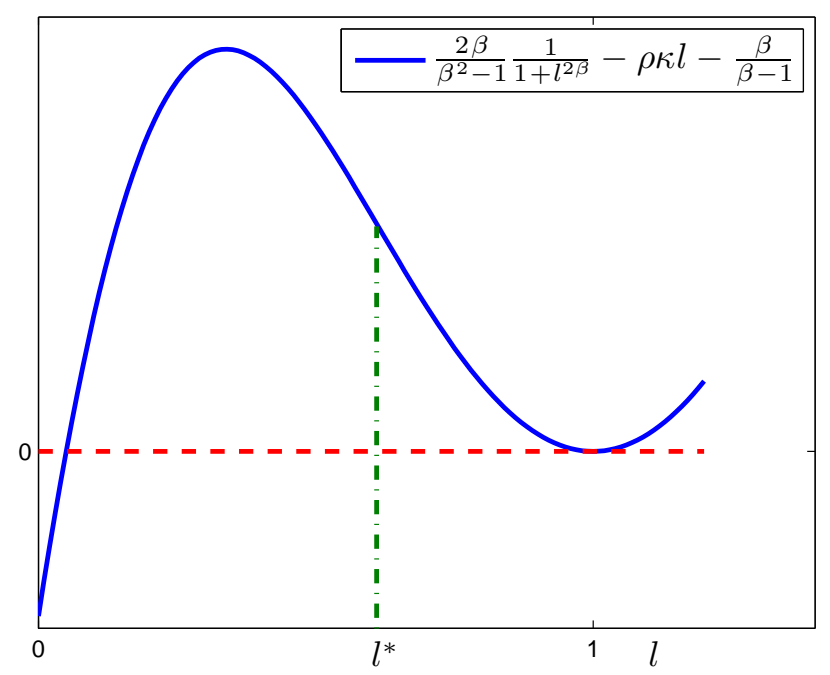

Figure 9: The left-hand side of (C.2)

Step 1. We verify that $l=1$ is a solution:

$$
\begin{aligned}
& \left.\left(\frac{2 \beta}{\beta^{2}-1} \frac{1}{1+l^{2 \beta}}-\rho \kappa l-\frac{\beta}{\beta-1}\right)\right|_{l=1}=\frac{\beta}{\beta^{2}-1}-\rho \kappa-\frac{\beta}{\beta-1} \\
= & \frac{-\beta^{2}}{\beta^{2}-1}-\rho \kappa=\frac{-\beta^{2}}{\beta^{2}-1}+\frac{\frac{2 \rho}{(\gamma \sigma)^{2}}}{\frac{2 \rho}{(\gamma \sigma)^{2}}-1}=0 .
\end{aligned}
$$

Step 2. The left-hand side of (C.2) is concave on $\left[0, l^{*}\right]$ and convex on $\left[l^{*}, \infty\right)$ where $l^{*}=\left(\frac{2 \beta-1}{2 \beta+1}\right)^{\frac{1}{2 \beta}} \in(0,1)$. (Figure 9 plots the left-hand side of (C.2).) To prove this, compute the first derivative of $\frac{1}{1+l^{2 \beta}}$ as $-\frac{2 \beta l^{2 \beta-1}}{\left(1+l^{2 \beta}\right)^{2}}$ and the second derivative as

$$
\frac{2 \beta l^{2 \beta-2}\left(1+l^{2 \beta}\right)}{\left(1+l^{2 \beta}\right)^{4}}\left((2 \beta+1) l^{2 \beta}-(2 \beta-1)\right) .
$$

Step 3. The slope of the left-hand side of (C.2) is zero at $l=1$ because

$$
\left.\left(\frac{2 \beta}{\beta^{2}-1} \frac{1}{1+l^{2 \beta}}-\rho \kappa l-\frac{\beta}{\beta-1}\right)^{\prime}\right|_{l=1}=-\left.\frac{2 \beta}{\beta^{2}-1} \frac{2 \beta l^{2 \beta-1}}{\left(1+l^{2 \beta}\right)^{2}}\right|_{l=1}-\rho \kappa=\frac{-\beta^{2}}{\beta^{2}-1}-\rho \kappa=0 .
$$

This implies that the equation (C.2) has no solution above one because it is convex above one.

Step 4. We show that (C.2) has a unique solution $l \in(0,1)$. The convexity of the left-hand side of (C.2) on $\left[l^{*}, 1\right]$ and the zero-slope condition shown in step 3 imply that

$$
\frac{2 \beta}{\beta^{2}-1} \frac{1}{1+l^{2 \beta}}-\rho \kappa l-\frac{\beta}{\beta-1}>0, \text { for all } l \in\left[l^{*}, 1\right) .
$$




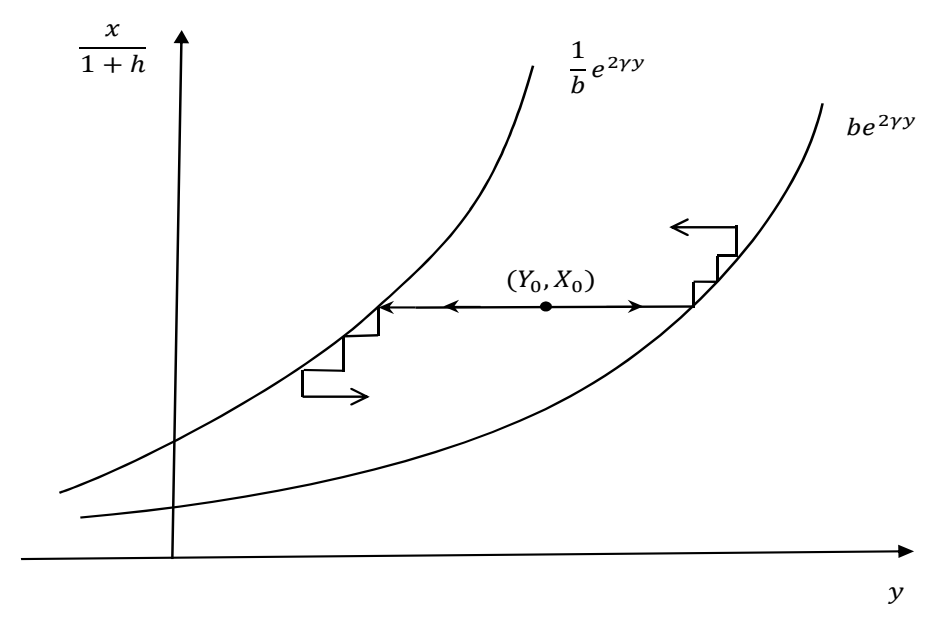

Figure 10: The state space for Example III. The two curves $\frac{x}{1+h}=b e^{2 \gamma y}$ and $\frac{x}{1+h}=\frac{1}{b} e^{2 \gamma y}$ partition the state space into three areas. The middle area is the no-jump region and the other two areas are the jump region.

Further, the left-hand side of (C.2) is below zero at $l=0$ because

$$
\left.\left(\frac{2 \beta}{\beta^{2}-1} \frac{1}{1+l^{2 \beta}}-\rho \kappa l-\frac{\beta}{\beta-1}\right)\right|_{l=0}=\frac{2 \beta}{\beta^{2}-1}-\frac{\beta}{\beta-1}=\frac{-\beta}{\beta+1}<0 .
$$

The Intermediate Value Theorem implies the existence of a solution $l \in(0,1)$. Next, we show the uniqueness of $l$. By contradiction, suppose there are two solutions, $0<l_{1}<l_{2}<l^{*}$. Because

$$
\begin{gathered}
\frac{2 \beta}{\beta^{2}-1} \frac{1}{1+l_{1}^{2 \beta}}-\rho \kappa l_{1}-\frac{\beta}{\beta-1}=0, \\
\frac{2 \beta}{\beta^{2}-1} \frac{1}{1+\left(l^{*}\right)^{2 \beta}}-\rho \kappa l^{*}-\frac{\beta}{\beta-1}>0,
\end{gathered}
$$

the concavity of the left-hand side of $(\mathrm{C} .2)$ on $\left[0, l^{*}\right]$ implies that

$$
\frac{2 \beta}{\beta^{2}-1} \frac{1}{1+l_{2}^{2 \beta}}-\rho \kappa l_{2}-\frac{\beta}{\beta-1}>0,
$$

which contradicts the fact that $l_{2}$ is a solution to (C.2). Q.E.D.

As in Example II, we rule out the degenerate solution. Figure 10 plots the state space. It shows that the two boundaries $x(1+h)^{-1}=b e^{2 \gamma y}$ and $x(1+h)^{-1}=b^{-1} e^{2 \gamma y}$ partition the state space into three areas. The area inside the two boundaries is the no-jump region and the other two areas are the jump region. The initial state $\left(Y_{0}, X_{0}^{*}\right)$ is inside the no-jump region. Figure 11 plots the dual value function $\tilde{V}(y, x, 0)$ and the primal value function $V(y, w)$ for three values $y \in\{-0.1,0,0.1\}$ in the no-jump region. This figure shows that $\tilde{V}(y, x, 0)$ is strictly 

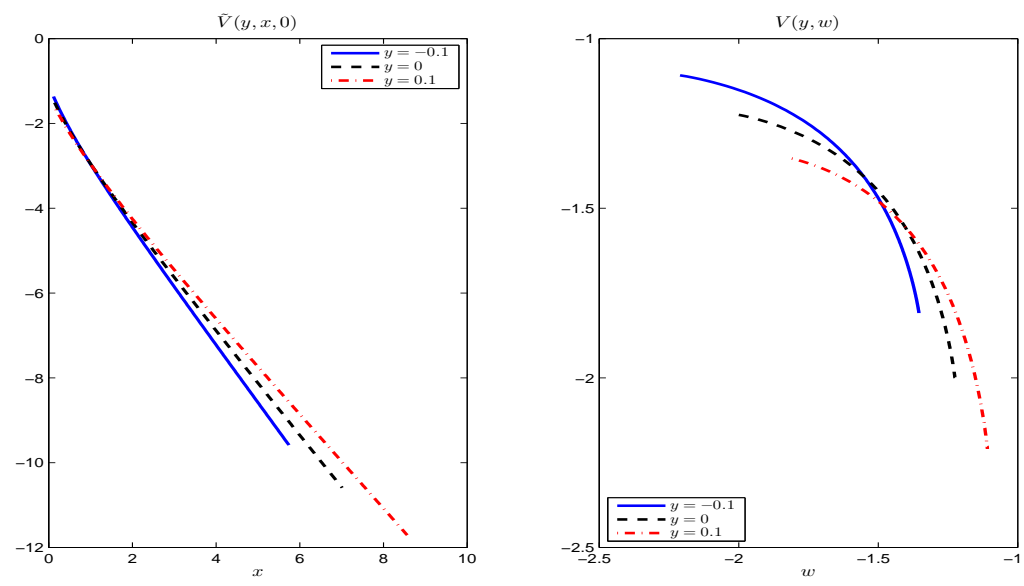

Figure 11: The dual and primal value functions in the no-jump region for Example III. Parameter values are given by $\rho=1, \gamma=1$, and $\sigma=1$.

convex in $x$ and $V(y, w)$ is strictly concave and decreasing in $w$. Note that both functions are non-monotonic with $y$ and the domains change with $y$. In particular, the domain of $V(y, w)$ for $w$ increases with $y$ because a larger promised value is needed to induce the agent's participation when his income is larger.

Figure 12 plots the simulated paths of incomes $Y_{t}$, consumption $C_{t}^{*}=(2 \gamma)^{-1} \ln \left(X_{t}^{*} / 1+H_{t}^{*}\right)$, the continuation value $W_{t}^{*}=V_{x}\left(Y_{t}, X_{t}^{*} /\left(1+H_{t}^{*}\right), 0\right)$, and $X_{t}^{*} /\left(1+H_{t}^{*}\right) e^{-2 \gamma Y_{t}^{*}}$. This figure shows intuitively how $C_{t}^{*}$ and $W_{t}^{*}$ move with incomes $Y_{t}$. Since

$$
C_{t}^{*}-Y_{t}=-Y_{t}+(2 \gamma)^{-1}\left(\ln X_{t}^{*}-\ln \left(1+H_{t}^{*}\right)\right)
$$

$C^{*}-Y$ is a regulated Brownian motion with drift zero and diffusion $-\sigma$ on $\left[\frac{1}{2 \gamma} \ln b,-\frac{1}{2 \gamma} \ln b\right]$. It follows from Proposition 5.5 in Harrison (1985) or Proposition 10.8 in Stokey (2008) that $C^{*}-Y^{*}$ has a unique stationary distribution which is uniform on $\left[\frac{1}{2 \gamma} \ln b,-\frac{1}{2 \gamma} \ln b\right]$.

Figure 13 presents comparative static results. As in Example II, the risk-sharing band $\left[\frac{1}{2 \gamma} \ln b,-\frac{1}{2 \gamma} \ln b\right]$ expands when one of the following cases happens: (i) the common coefficient of relative risk aversion rises, (ii) the volatility of the income process rises, or (iii) the common subjective discount rate falls. In addition, neither autarky nor the first-best allocation is an optimal contract for any admissible parameter values satisfying the assumption in Proposition 3. The intuition is also similar to that for Example II. 

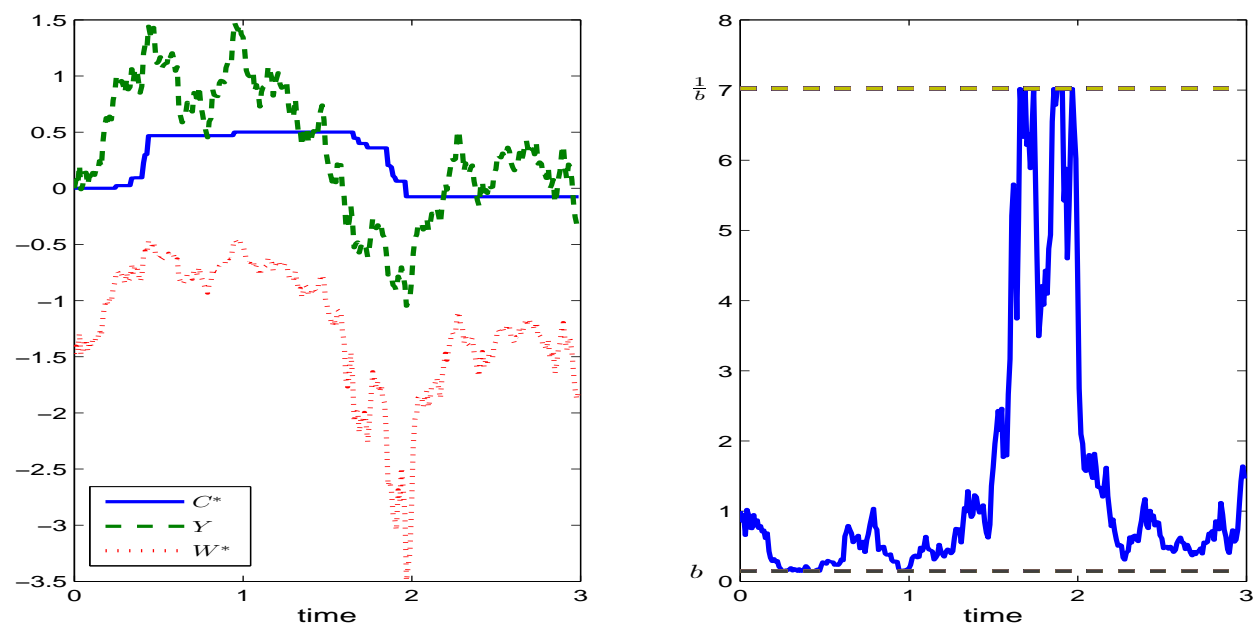

Figure 12: Simulated paths of the agent's optimal consumption $C_{t}^{*}$, incomes $Y_{t}$, continuation values $W_{t}^{*}$, and the process $e^{-2 \gamma Y_{t}} X_{t}^{*} /\left(1+H_{t}^{*}\right), t \geq 0$, for Example III. Parameter values are given by $\rho=1, \gamma=1$, and $\sigma=1$.
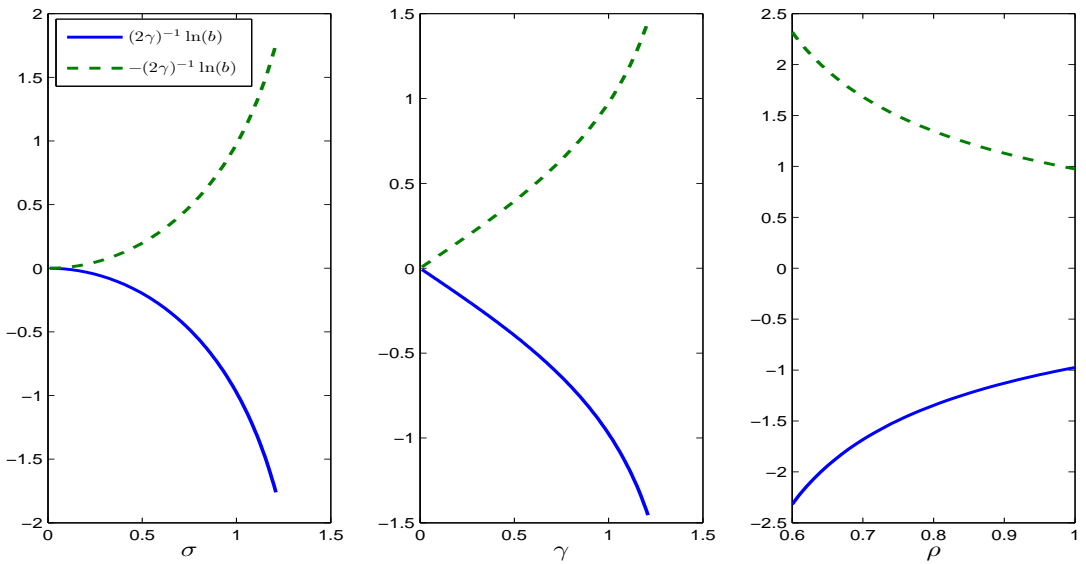

Figure 13: Comparative statics for Example III. Parameter values are given by $\sigma=1, \rho=1$, and $\gamma=1$, unless one of them is changed in the comparative statics. 


\section{References}

Abreu, D., D. Pearce, and E. Staccheti (1990): "Toward a Theory of Discounted Repeated Games with Imperfect Monitoring," Econometrica, 58(5), 1041-1063.

Acemoglu, D., M. Golosov, and A. Tsyvinski (2011): "Political Economy of Ramsey Taxation," Journal of Public Economics, 95(7-8), 467-475.

Alvarez, F., And U. Jermann (2000): "Efficiency, Equilibrium, and Asset Pricing with Risk of Default," Econometrica, 68(4), 775-797.

Alvarez, F., and U. J. Jermann (2001): "Quantitative Asset Pricing Implications of Endogenous Solvency Constraints," Review of Financial Studies, 14(4), 1117-51.

Bergemann, D., and P. Strack (2014): "Dynamic Revenue Maximization: A Continuous Time Approach," Cowles Foundation Discussion Papers 1953, Cowles Foundation for Research in Economics, Yale University.

Biais, B., T. Mariotti, G. Plantin, and J.-C. Rochet (2007): "Dynamic Security Design: Convergence to Continuous Time and Asset Pricing Implications," Review of Economic Studies, 74(2), 345-390.

Bismut, J.-M. (1973): "Conjugate Convex Functions in Optimal Stochastic Control," Journal of Mathematical Analysis and Applications, 44(2), 384-404.

Bulow, J., And K. Rogoff (1989): "Sovereign Debt: Is to Forgive to Forget?" American Economic Review, 79(1), 43-50.

Chari, V. V., and P. J. Kehoe (1993): "Sustainable Plans and Mutual Default," Review of Economic Studies, 60(1), 175-95.

Cochrane, J. H. (1991): "A Simple Test of Consumption Insurance," Journal of Political Economy, 99(5), 957-76.

Cooley, T., R. Marimon, and V. Quadrini (2004): "Aggregate Consequences of Limited Contract Enforceability," Journal of Political Economy, 112(4), 817-847.

DeMarzo, P. M., and Y. Sannikov (2006): "Optimal Security Design and Dynamic Capital Structure in a Continuous-Time Agency Model," Journal of Finance, 61(6), 2681-2724.

Duffie, D. (1996): Dynamic Asset Pricing Theory, Second Edition. Princeton University Press. 
Dumas, B. (1991): "Super Contact and Related Optimality Conditions," Journal of Economic Dynamics and Control, 15(4), 675-685.

Fleming, W., And H. Soner (2006): Controlled Markov Processes and Viscosity Solutions, Second Edition. Springer Science+ Business Media, Inc., New York, NY, USA.

Green, E. J. (1987): "Lending and the Smoothing of Uninsurable Income," in Contractual Arrangements for Intertemporal Trade, ed. by E. C. Prescott, and N. Wallace, pp. 3-25. University of Minnesota Press, Minneapolis.

Grochulski, B., And Y. Zhang (2011): "Optimal Risk Sharing and Borrowing Constraints in a Continuous-time Model with Limited Commitment," Journal of Economic Theory, 146(6), $2356-2388$.

Hansen, L., D. Epple, and W. Roberds (1985): "Linear-Quadratic Duopoly Models of Resource Depletion," in Energy, Foresight, and Strategy, ed. by T. J. Sargent. Johns Hopkins University Press.

Harrison, M. (1985): Brownian Motion and Stochastic Flow Systems. Wiley, New York.

Harrison, M., and M. I. Taksar (1983): "Instantaneous Control of Brownian Motion," Mathematics of Operations Research, 8(3), 439-453.

He, H., And H. F. Pages (1993): "Labor Income, Borrowing Constraints, and Equilibrium Asset Prices," Economic Theory, 3(4), 663-96.

Hellwig, C., And G. Lorenzoni (2009): "Bubbles and Self-Enforcing Debt," Econometrica, $77(4), 1137-1164$.

Kehoe, P., and F. Perri (2002): "International Business Cycles with Endogenous Incomplete Markets," Econometrica, 70(3), 907-928.

Kehoe, T., and D. Levine (1993): "Debt-Constrained Asset Markets," Review of Economic Studies, 60(4), 865-888.

Kletzer, K. M., And B. D. Wright (2000): "Sovereign Debt as Intertemporal Barter," American Economic Review, 90(3), 621-639.

Kocherlakota, N. (1996): "Implications of Efficient Risk Sharing without Commmitment," Review of Economic Studies, 63(4), 595-609.

- (2008): "Injecting Rational Bubbles," Journal of Economic Theory, 142(1), 218-232. 
Krueger, D., And F. Perri (2006): "Does Income Inequality Lead to Consumption Inequality? Evidence and Theory," Review of Economic Studies, 73(1), 163-193.

(2011): "Public versus Private Risk Sharing," Journal of Economic Theory, 146(3), 920-956.

Krueger, D., And H. Uhlig (2006): "Competitive Risk Sharing Contracts with One-sided Commitment," Journal of Monetary Economics, 53(7), 1661-1691.

Kydland, F. E., and E. C. Prescott (1980): "Dynamic Optimal Taxation, Rational Expectations and Optimal Control," Journal of Economic Dynamics and Control, 2(1), 79-91.

Ligon, E., J. Thomas, and T. Worrall (2002): "Informal Insurance Arrangements with Limited Commitment: Theory and Evidence from Village Economies," Review of Economic Studies, 69(1), 209-44.

Ljungqvist, L., And T. Sargent (2004): Recursive Macroeconomic Theory, Second Edition. The MIT Press, Cambridge, Massachusetts, USA.

Mace, B. J. (1991): "Full Insurance in the Presence of Aggregate Uncertainty," Journal of Political Economy, 99(5), 928-56.

Marcet, A., and R. Marimon (1992): "Communication, Commitment, and Growth," Journal of Economic Theory, 58(2), 219-249.

— (1998): "Recursive Contracts," European University Institute, Working Paper.

Messner, M., N. Pavoni, and C. Sleet (2011): "On the Dual Approach to Recursive Optimization," GSIA Working Papers 2012-E8, Carnegie Mellon University, Tepper School of Business.

- (2012): "Recursive Methods for Incentive Problems," Review of Economic Dynamics, $15(4), 501-525$.

Miao, J., And A. Rivera (2013): "Robust Contracts in Continuous Time," Boston University - Department of Economics.

Miao, J., And P. Wang (2011): "Bubbles and Credit Constraints," Boston University Department of Economics - Working Papers Series, WP2011-031.

- (2012): "Bubbles and Total Factor Productivity," American Economic Review, 102(3), $82-87$. 
Pavan, A., I. Segal, and J. Tolkka (2014): "Dynamic Mechanism Design: A Myersonian Approach," Econometrica, 82(2), 601-653.

Sannikov, Y. (2008): "A Continuous-Time Version of the Principal-Agent Problem," Review of Economic Studies, 75(3), 957-984.

(2012): "Moral Hazard and Long-Run Incentives," Princeton University - Department of Economics.

Spear, S., and S. SRIvastava (1987): "On Repeated Moral Hazard with Discounting," Review of Economic Studies, 54(4), 599-617.

Stokey, N. (2008): The Economics of Inaction: Stochastic Control Models with Fixed Costs. Princeton University Press, New Jersey.

Strulovici, B. (2011): "Contracts, Information Persistence, and Renegotiation," Northwestern University - Department of Economics.

Thomas, J., And T. Worrall (1988): "Self-Enforcing Wage Contracts," Review of Economic Studies, 55(4), 541-554.

Townsend, R. M. (1994): "Risk and Insurance in Village India," Econometrica, 62(3), 539-91.

Williams, N. (2009): "On Dynamic Principal-Agent Models in Continuous Time," Working paper, University of Wisconsin-Madison.

— (2011): "Persistent Private Information," Econometrica, 79(4), 1233-1275.

Xu, G.-L., And S. E. Shreve (1992): "A Duality Method for Optimal Consumption and Investment Under Short-Selling Prohibition. I. General Market Coefficients," The Annals of Applied Probability, 2(1), 87-112.

Zhang, Y. (2013): "Characterization of a Risk Sharing Contract with One-sided Commitment," Journal of Economic Dynamics and Control, 37(4), 794-809. 